\title{
NON-TRACKED MIRROR-AUGMENTED PHOTOVOLTAIC DESIGN AND PERFORMANCE
}

BY

\author{
WEI-CHUN LIN
}

Submitted in partial fulfillment of the requirements

For the degree of Master of Science

Thesis Advisor: Dr. Roger H. French

Department of Material Science and Engineering

CASE WESTERN RESERVE UNIVERSITY

MAY, 2013 


\section{Non-tracked Mirror-Augmented Photovoltaic Design and Performance \\ CASE WESTERN RESERVE UNIVERSITY \\ SCHOOL OF GRADUATE STUDIES}

We hereby approve the thesis/dissertation of

Wei-Chun Lin

candidate for the Master of Science degree *

Dr. Roger H. French

(Committee Chair, Adviser)

Signature/Date

Dr. Mark R. DeGuire

(Committee Member)

Signature/Date

Dr. David Matthiesen

(Committee Member)

Signature/Date

(date)

Dec. 182012

*We also certify that written approval has been obtained for any proprietary material contained therein. 
Dedicated to my beloved parents 


\section{Table of Contents}

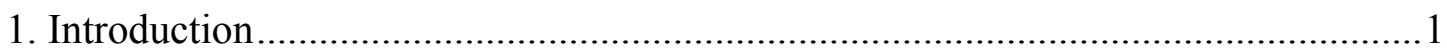

1.1 The Concept of Mirror-Augmented Photovoltaic (MAPV)............................

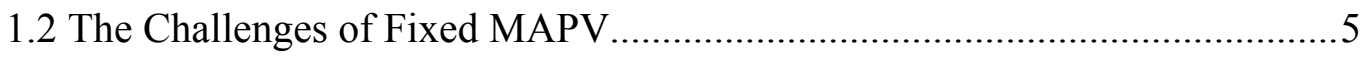

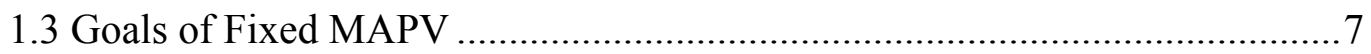

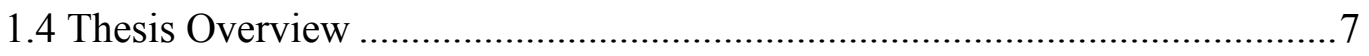

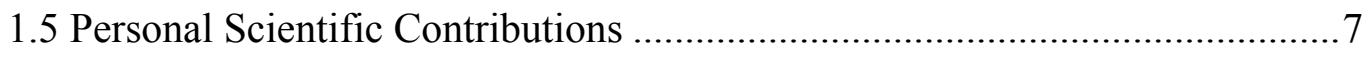

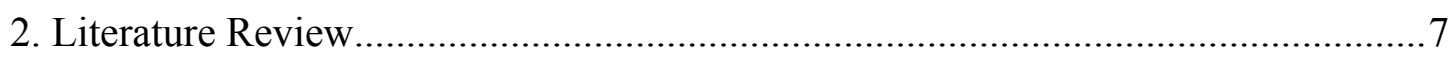

2.1 A Historical Review of Low Concentration PV ............................................

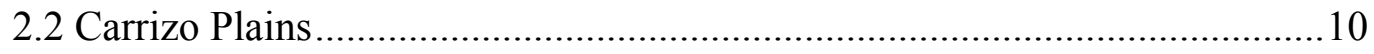

2.2.1 PV Module Design- Front Glass................................................................14

2.2.2 PV Module Design- Encapsulation Materials...............................................15

2.2.3 Important Factors of Degradation Mechanisms at Carrizo Plains ..............16

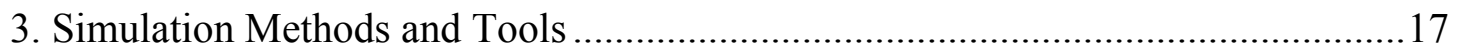

3.1 1D Model for Annual Irradiance by MatLab ………....................................17

3.2 3D Geometric Optical Analysis by TracePro ${ }^{\circ}$...............................................21 
3.2.2 The Definition of Material and Surface Properties .23

3.2.3 The Simulation Process. 25

3.3 Outdoor Experimental Data Confirm with Model Simulation 26

3.4 Determination of the Date at Outdoor Field-Test Site 27

3.5 Sample Nomenclature of Fixed MAPV Model .28

4. Results 29

4.1 Model Configuration for Yearly Irradiance Output 29

4.2 Types of Irradiance Non-Uniformity for Fixed MAPV Models 30

4.3 Comparison of 3D Models and Field-Test Data...... 38

4.4 Power Production from Fixed MAPV Over a 3 Month Period . .40

4.5 Performance on Annual Power Production .42

4.6 The Effect of Mirror Tilt Angle on Uniformity and Irradiance Value .44

4.7 Possible Solutions for the "Gullwing Curve". .49

5. Discussion 52

5.1 Maximum Yearly Power Production for Fixed MAPV 52

5.2 Mitigation of Non-Uniform Irradiance in Fixed MAPV 53 
5.3 Power Production Improvement of Fixed MAPV for Field-Tests.

5.4 Differences between Snapshot Power Production and Field-Test Power

Production

5.5 A Gullwing Curve on Annual Power Production

5.6 The Effect of Mirror Tilt Angle on Uniformity and Power Production 58

5.7 Viability of Different Mirror Designs .60

6. Conclusion .61

Bibliography .63 


\section{List of Figures}

Figure 1: The concept of mirror-augmented photovoltaic (MAPV), which solar mirrors are used to reflect additional light onto a PV module. 3

Figure 2: Tracked MAPV system, a) a dual-axis tracker with non-MAPV system, b) mirror-augmented samples and modules were exposed to various intensities of solar irradiance at SDLE SunFarm (Cleveland, OH). 4

Figure 3: The fixed MAPV test system at Replex Plastics.............................................. 4

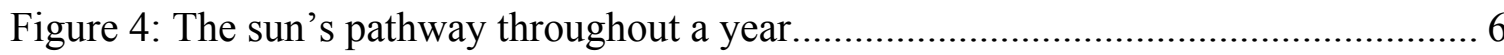

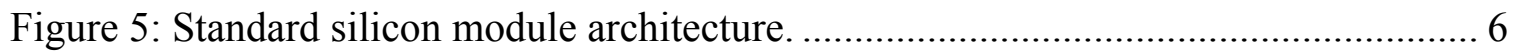

Figure 6: An image of the mirror-augmented tracker at Carrizo Plains. .......................... 10

Figure 7: Power degradation of the Carrizo Plains compared to typical degradation rates for current flat-panel PV modules ${ }^{19}$ 11

Figure 8: Representative modules of block I through block V (from left to right) ${ }^{22} \ldots \ldots . .12$

Figure 9: The discoloration of EVA specimen after exposure to 42 suns cover by Ce glass (left) and non-Ce glass (right) ${ }^{23}$. 14

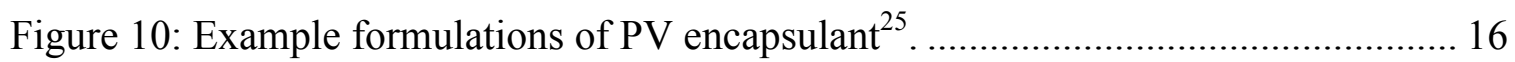

Figure 11. The sketch of mirror throw distance on MAPV architecture. ........................ 20

Figure 12: A 3D MAPV model constructed by commercial CAD software, SolidWorks ${ }^{\circledR}$. A small gap was designed to prevent the module from dirt or snow accumulation. 23 
Figure 13: The 3D MAPV model was imported into TracePro ${ }^{\circledR}$ and surface properties were defined

Figure 14: The MAPV model with different rectangular grid sources 25

Figure 15: The ray-trace result from TracePro ${ }^{\circledR}$, which contains (a) light distribution on PV panel and (b) line profile. 26

Figure 16: Estimated yearly irradiance versus module tilt angle for a number of mirror tilt angles. 30

Figure 17: A 3D geometric model created by a commercial CAD software, SolidWorks. The PV module tilt angle is $50^{\circ}$ and the mirror tilt angle is $10^{\circ}$ (Gen2.50.10)....... 31

Figure 18: The ray-trace result of Gen2.50.10 model (a) and the irradiance map from model for the sun's position at 8 a.m. (b) 32

Figure 19: The irradiance maps of Gen2.50.10 model at 9 a.m. (a) and 10 a.m. model (b). 33

Figure 20: The irradiance maps of Gen2.50.10 model at 11 a.m. (a) and 12 p.m. (b)..... 33

Figure 21: The irradiance maps of Gen2.50.10 model at 1 p.m. (a) and 2 p.m. (b)........ 34

Figure 22: The irradiance maps of Gen2.50.10 model at 3 p.m. (a) and 4 p.m. (b)........ 35

Figure 23: The light distribution on a module (Gen2.50.10) throughout a day from 8 a.m.

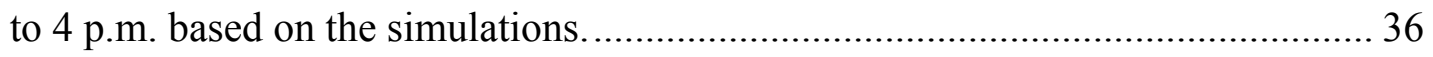


Figure 24: A 3D geometric model (Gen2.50.10) in optical analysis, where z-axis represents north, $\mathrm{x}$-axis represents east/west direction. 36

Figure 25: Ray-trace result from 12 p.m. panel, the red line represents incident rays and the blue line represents the reflected rays.

Figure 26: A 3D geometric model with $3 \mathrm{X}$ wide mirror, the $\mathrm{X}$-axis represents the eastwest direction. 37

Figure 27: The irradiance maps of the $3 \mathrm{x}$ wide mirror model through one day from 8 a.m. to 4 p.m. 38

Figure 28: Average irradiance comparisons between MAPV and non-MAPV from raytrace result. 39

Figure 29: I-V curve result from the field-test site at Replex Plastics. The inset irradiance map shows where the by-pass diodes operate. 40

Figure 30: The fixed MAPV test system at Replex Plastics (latitude: 40.38, longitude: 82.48).

Figure 31: The prototype of time machine apparatus. 42

Figure 32: Relative position between the $3 \mathrm{X}$ wide mirror model and the sun from the $15^{\text {th }}$ of each month. 43

Figure 33: Estimated power output of Gen2.50.10 model by month 44

Figure 34: Ray-trace result of Gen2.55.25 on July $15^{\text {th }}$ shows uniform irradiance distribution (a) and its light pathway (b). 46 
Figure 34: The ray-tracing results of Gen2.55.30 on July $15^{\text {th }}$, which shows a nonuniform region occurring on the PV panel (a) and the pathway of light (b).

Figure 36: Ray-trace result of Gen2.55.35 on July $15^{\text {th }}$ shows a significant non-uniform region on the panel (a) and the pathway of light (b). 48

Figure37: The irradiance values throughout a year from various model configurations.. 49

Figure 38: A new MAPV design of a barrel mirror with (a) 90 in. radius of curvature, and (b) with a 540 in. radius of curvature. 50

Figure 39: The ray-trace result of the Gen3.55.0.90 model at noon on January $10^{\text {th }}, 2012$,

(a) irradiance map and (b) the pathway of light................................................ 50

Figure 40: The Ray-trace result of the Gen3.55.0.540 model at noon on January $10^{\text {th }}$, 2012 (a) irradiance map and (b) the pathway of light.......................................... 51

Figure 41: The ray-trace result of the Gen4.55.10.100 model at noon on March $10^{\text {th }}, 2012$ (a) irradiance map and (b) the pathway of light............................................. 51

Figure 42: The ray-trace result of the Gen4.55.10.900 model at noon on March $10^{\text {th }}, 2012$ (a) irradiance map and (b) the pathway of light. 52

Figure 43: The ray-trace result for the Gen2.50.10 model during summer. 58

Figure 43: The ray-trace result for the Gen2.55.30 model on June $15^{\text {th }}, 2012$ at noon. ... 60 


\section{List of Tables}

Table 1: Representative characteristics of block modules ${ }^{22}$.

Table 2: Sun's intensities throughout a day

Table 3. The studied model configurations and its experimental tools 28

Table 4: Power production of each month at field-test site* 41

Table 5: A series simulation of mirror tilt angle and its performance on the irradiance map and the average irradiance value $\left(\mathrm{W} / \mathrm{m}^{2}\right)$ 


\section{Acknowledgement}

This research project would not have been possible without the support of people from CWRU SDLE Center and Replex Plastics Inc.

The author would like to express his sincerest appreciation to:

Professor Mark R. DeGuire

Professor David Matthiesen

Dave Hollingshead

Scott A. Brown

Kara A. Shell

Mark Schuetz

Myles P. Murray

Dr. Laura S. Bruckman

and other group members in the lab. 
Non-tracked Mirror-Augmented Photovoltaic Design and Performance

Abstract

By

\section{WEI-CHUN LIN}

In developing photovoltaic (PV) technology, it is crucial to provide low cost PV power. One of the useful methods is to increase the power output of conventional PV modules since the major cost (module manufacturing, mounts, wiring, installation labor, etc.) tends to scale with system area. Increased power output, due to improved light harvesting, will produce more power per unit area. The use of PV modules, which have been augmented by the addition of low-cost solar mirrors, provides the opportunity to improve light harvesting of PV modules while reducing the cost of power. In order to harvest more incident solar irradiance, an optimized design configuration between a flatpanel module and mirror is necessary for a fixed (non-tracked) mirror-augmented photovoltaic (MAPV) system. A series of 1D irradiance models were developed to screen various MAPV design configurations. Optical ray-tracing was used to determine irradiance non-uniformity issues on a fixed MAPV system. Both the 1D irradiance and ray-tracing results are compared to outdoor field test results. The current-voltage (I-V) curve tracing of test modules was performed with a Daystar Multi-tracer for time series analysis. Over a three-month period of study the fixed MAPV system produced $10 \%$ more power than an equivalent non-augmented panel. An adjustable mounting system "time machine" was used to estimate yearly power production. The experimental time machine result matched the ray-trace simulation and showed a "gullwing curve" of 
monthly power output with peak production on the equinoxes and reduced production on the solstices. This "gullwing" power production is characteristic for fixed MAPV system geometrics. 


\section{Introduction}

Solar energy, radiant light and heat from the sun, has been harnessed by human beings for centuries. As early as the $7^{\text {th }}$ century B.C., people used simple magnifying glasses to concentrate the sunlight into beams in order start cooking fires ${ }^{1}$. In the 1900 s, scientists began seriously investigating methods to harness solar energy. Solar technologies are broadly characterized as either active solar or passive solar depending on the way these technologies capture, convert and distribute solar energy. Passive solar techniques include orienting a building to the sun, selecting materials with favorable thermal mass or light dispersing properties and designing spaces that naturally circulate air. Active solar techniques include the use of photovoltaic panels and solar thermal collectors to harness the energy. The solar technology grew until the mid-1950s, when low-cost petroleum became the primary fuel for modern society. In recent years, the enormous increase in global demand for energy in past decades was caused by industrial development and population growth. The supply of energy has not increased sufficiently to meet the actual demand. Because the current energy crisis has greatly increased the cost of fossil fuels ${ }^{2}$, replacement energy sources are drawing much more attention than previously. Solar energy is among those alternative energy sources that have been in the spotlight. Nevertheless, it is not easy for solar energy to compete with other low-cost energy sources such as nuclear, thermal and wind power in geographic areas of low solar insolation, such as the northern United States ${ }^{3}$. Therefore, solar technology needs to increase its efficiency in order to lower the cost. 


\subsection{The Concept of Mirror-Augmented Photovoltaic (MAPV)}

One solution to make solar energy more competitive is to combine reflectors with the PV modules in the system. Using solar mirrors to harvest more of the incident solar irradiance and direct sunlight to qualified PV modules increases the electricity produced from a given area of PV panels ${ }^{4}$. Replex Plastics Inc., which is a supplier of high impact, high performance optical domes, mirrors and custom thermoformed plastic parts, has developed a high performance, low-cost solar back surface mirror made of poly (methyl methacrylate) (PMMA) acrylic ${ }^{5}$. The acrylic mirror uses a back surface aluminum reflector and is ideal for PV augmentation because of its low cost, lightweight (at typical $3 \mathrm{~mm}$ thickness, solar mirror weighs $3.6 \mathrm{~kg} / \mathrm{m}^{2}$ ) and low light scattering (compared to polished glass mirror $)^{6}$. The back surface solar mirror, can spectrally filter ultra-violet (UV) light which prevents the PV panel from experiencing additional harmful UV irradiance reflected from the mirror ${ }^{7,8,9,10}$. Therefore, mirror-augmented photovoltaic (MAPV) systems can improve harvesting of a fixed $1 \mathrm{~kW} / \mathrm{m}^{2}$ solar resource and make solar energy competitive with other energy systems. Figure 1 illustrates the concept of MAPV. 


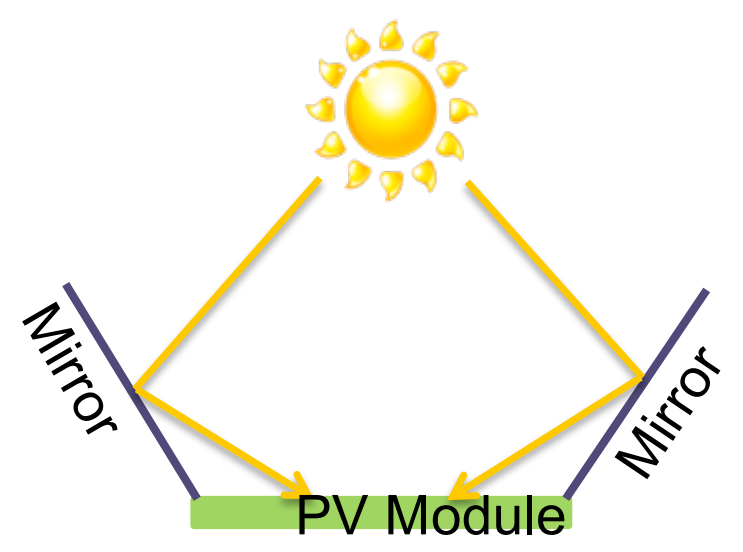

Figure 1: The concept of mirror-augmented photovoltaic (MAPV), which solar mirrors are used to reflect additional light onto a PV module.

In general, the MAPV system can be divided into two categories: tracked MAPV and non-tracked MAPV. In a tracked MAPV system, the MAPV modules are mounted on trackers that track the sun as it moves through out the day and the year. Figure 2a shows an image of PV modules mounted on tracker at the SunFarm of the Solar Durability and Lifetime Extension (SDLE) Center and Figure 2b demonstrates the sketch of mirroraugmented PV and samples on tracker. 

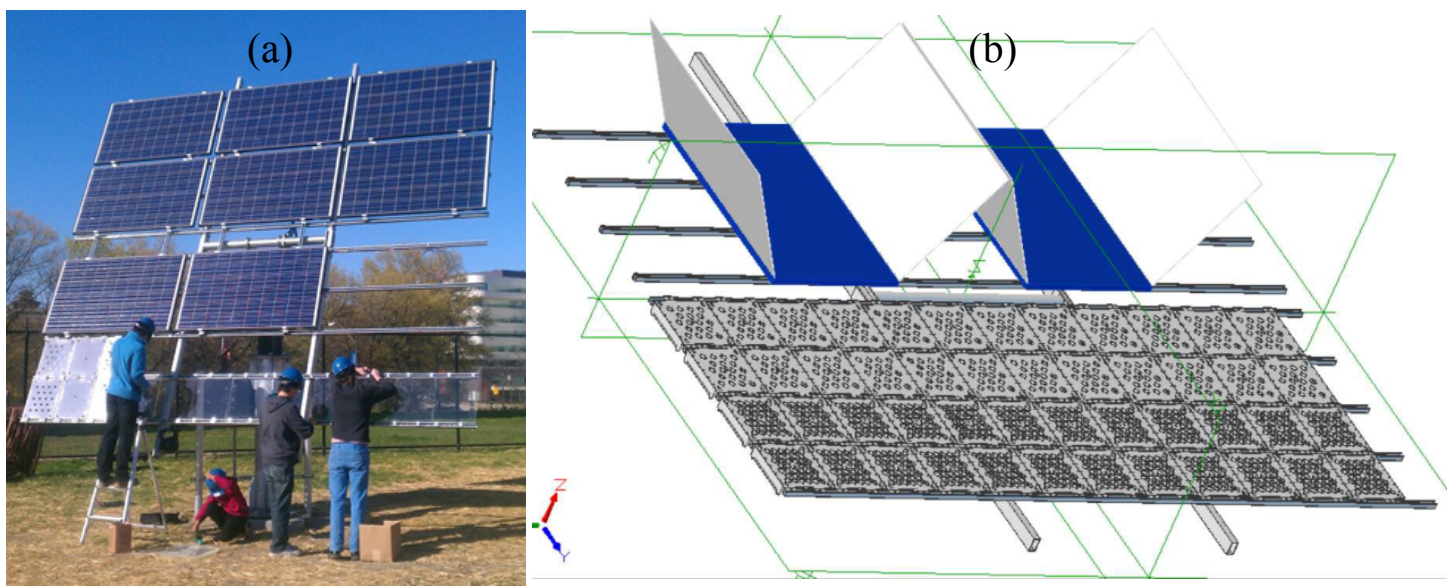

Figure 2: Tracked MAPV system, a) a dual-axis tracker with non-MAPV system, b) mirror-augmented samples and modules were exposed to various intensities of solar irradiance at SDLE SunFarm (Cleveland, OH).

The non-tracked MAPV or fixed MAPV system, which has MAPV modules mounted on a fixed metal frame without the ability to track the pathway of the sun. Figure 3 shows an image of the fixed MAPV construction installed at Replex Plastics test site near Columbus, OH. A row of three consecutive PV panels was coupled with acrylic solar mirrors. The area ratio of the mirror to PV module is 1:1.

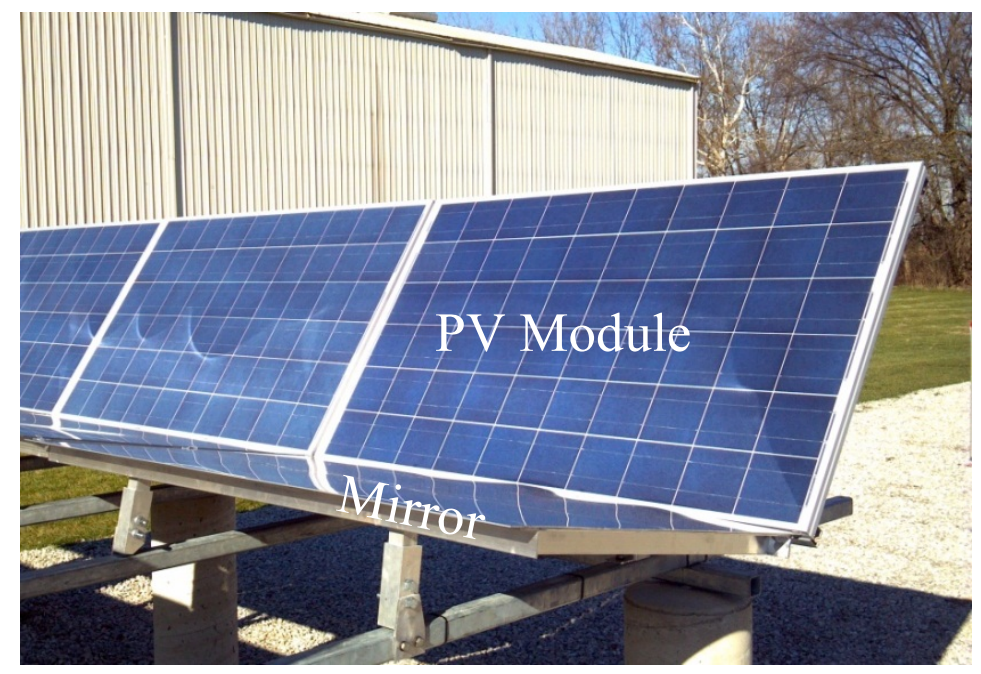

Figure 3: The fixed MAPV test system at Replex Plastics. 


\subsection{The Challenges of Fixed MAPV}

A fixed MAPV system still has challenges, which need to be solved in order for MAPV to become a valid low cost option for the PV industry. One of the challenges with fixed MAPV is maintaining even illumination on a module because the sun's position is constantly moving throughout the year, which leads to possible alignment issues with the fixed position of the module and mirrors. Figure 4 shows the sun's pathway during a year, which shows that the sun's rays are hitting the MAPV system at different angles. For example, the sun's elevation angle is low in the winter and is high in the summer. The easiest solution is to place PV modules and mirrors on a solar tracker, ensuring a constant illumination pattern. Although the tracked MAPV system can harvest more light, the cost is also increased greatly. A fixed MAPV system offers a low-cost solution, but introduces irradiance non-uniformity issues. The non-uniformity issue in crystalline silicon-based fixed MAPV modules cause power lost due to by-pass diodes operating. The challenge of fixed MAPV is optimizing the system properly to maximize annual power production. 


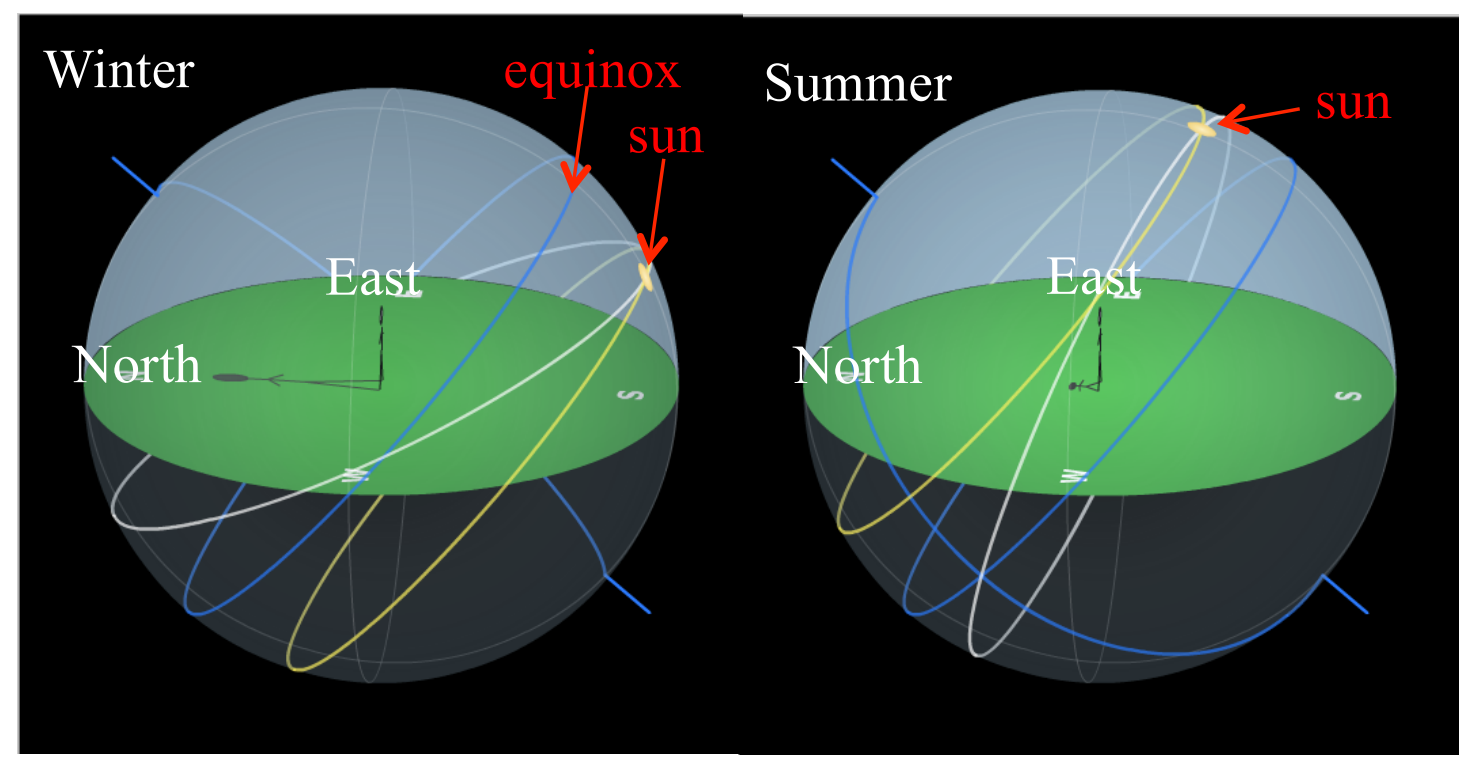

Figure 4: The sun's pathway throughout a year ${ }^{11}$.

Another challenge of the fixed MAPV is the irradiance non-uniformity. The cell string architecture of a standard crystalline silicon (c-Si) PV module (Figure 5) is not ideal for MAPV, due to the inherently non-uniform irradiance. The by-pass diodes are operating while non-uniformity occurs and it leads to a loss in enhanced power from the mirror-augmented area. Dealing with the irradiance uniformity is a primary concern for the system ${ }^{12}$.

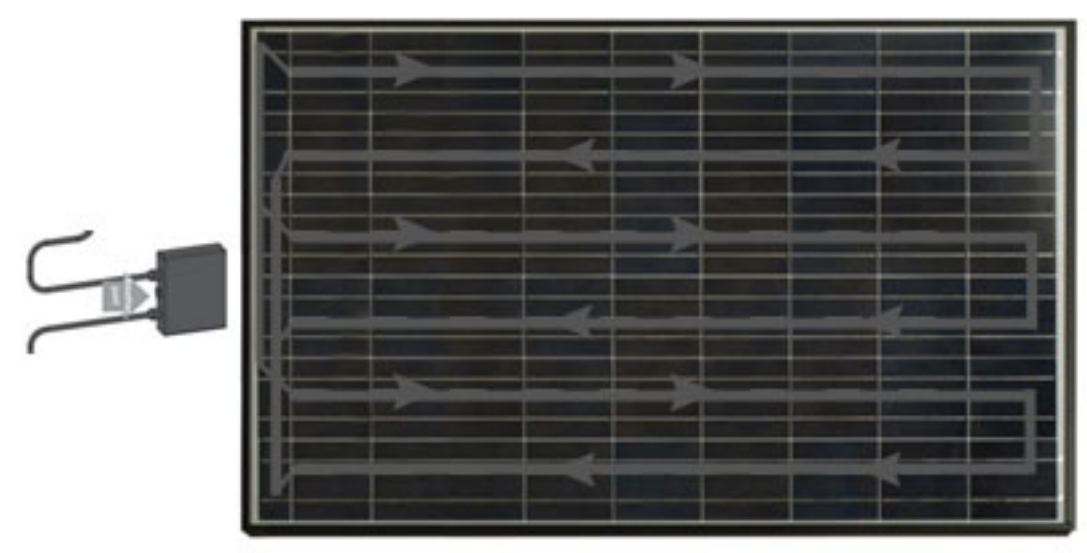

Figure 5: Standard silicon module architecture. 


\subsection{Goals of Fixed MAPV}

Hence, the goals for fixed MAPV with c-Si PV modules is to obtain the most uniform irradiance distribution possible while producing maximum annual power.

\subsection{Thesis Overview}

A series of $1 \mathrm{D}$ calculations were developed to determine the maximum power production over an entire year. The $3 \mathrm{D}$ geometric analysis was used to observe the irradiance uniformity of PV panels. The outdoor field test results are compared to indoor simulations. An adjustable mounting system "time machine" was used to estimate yearly power production.

\subsection{Personal Scientific Contributions}

Personal contributions to this research work were extensive. Firstly, finding out the accurate sun's position throughout a day and ray-tracing the sun with different models. After the first prototype of MAPV was designed, a series of simulations were investigated. Further designs of MAPV were used to observe the irradiance distribution of MAPV models. Continual data analysis between simulations and power output from field-test site led to publication of this thesis.

\section{Literature Review}

Amplification of solar irradiance on crystalline silicon PV modules using mirrors was seen as a possible solution for a high efficient energy source. The concentrated PV (CPV) systems are categorized according to the amount of their solar concentration, 
measured in "suns". Low concentration PV (LCPV) are systems with a solar concentration of 1-10X suns. In most LCPV cases, a tracking system is not required. Medium concentration PV are systems with 12-100X suns concentration, which requires 1- or 2-axis trackers and cooling systems. High concentration PV (HCPV) concentrates sunlight to intensities of 200-1000X suns. The HCPV requires high-capacity heat sinks to prevent thermal destruction and to manage temperature related performance losses. In this thesis, the research topic focuses on LCPV where non-tracked PV modules are combined with reflectors for the 1-10X concentration of solar radiation. A brief history review of LCPV will be introduced. A prominent example related to mirror-augmented PV is the Carrizo Plains installation. The degradation mechanisms of MAPV modules at Carrizo Plains will be discussed in this section.

\subsection{A Historical Review of Low Concentration PV}

Early solar thermal electric systems used concentrators to generate the high temperature needed for efficient conversion. These systems basically relied on reflective concentrators, which could be obtained from the mature glass mirror technology. K. Butti mentioned a solar electric system built in Egypt in 1913 using reflective troughs and reportedly attained a conversion efficiency of 3-4\% from sunlight to electricity ${ }^{13}$. In 1952, Bell Laboratories demonstrated a silicon solar cell and developed it into a practical device with $10 \%$ conversion efficiency by $1955^{14}$. The high cost of the cell precluded its use as a primary source of terrestrial energy, except in small, remote applications.

Work began on PV systems shortly thereafter to reduce cost of modules. In the early 1960s, the Wisconson Solar Energy Center investigated the performance of solar cells under concentrated sunlight ${ }^{15}$. It was shown that the cells could operate at intensities of 
several hundred times that of normal sunlight ${ }^{16}$. Then, the Wisconson group went on to design a working $50 \mathrm{~W}$ system using a parabolic dish concentrator, thereby demonstrating the feasibility of the concept ${ }^{17}$. In 1965, Eugene Ralph proposed several approaches for concentrating systems, from low concentration reflective cones to high concentration heliostat fields. He clearly articulated the vision that CPV systems could be built in the future for less than $\$ 5.00 / \mathrm{W}$ in current dollars ${ }^{15}$. Despite these early efforts, not much happened in the development of practical concentrating systems until the oil crisis of the 1970s. As crystalline silicon became the utility scale PV technology of choice, it became critical to optimize systems on many levels, including the metrics of dollars invested per watt peak production and levelized cost of energy (LCOE), which is the price of the system over its life divided by the total predicted output of the system. Both of these metrics can be lowered significantly by mirror enhancement of PV modules. By focusing the sun's energy onto a PV array with low-cost mirror systems, it is possible to increase both the peak power output and lower the cost of energy. Because mirrors cost less than a dime on the dollar when compared with PV modules, there is the possibility for significant savings on LCOE by utilizing MAPV. This value proposition rests in the notion that mirror augmentation will not decrease the life of PV modules due to increased UV degradation or increased temperature caused by increased infrared dosing. In addition, mirrors need to be designed and manufactured such that they can achieve long lifetimes when exposed to environmental exposure. As such MAPV needs to be proven to be safe for modules and cost effective before it can capture significant market share ${ }^{15}$. 


\subsection{Carrizo Plains}

The world's first utility scale PV power plant, the Carrizo Plains, came online in late $1983^{18}$. The facility was owned by Arco Solar, Inc. until late 1989 when it was purchased by Carrizo Solar Corporation. The Carrizo Plains power plant consisted of 10 segments, each with its own power conditioning unit (PCU). Segments 1 through 9 had a combined total of 756 trackers with mirrors in a v-trough configuration (Figure 6) providing nominal 2:1 concentration, while segment 10 had 43 trackers without mirrors.

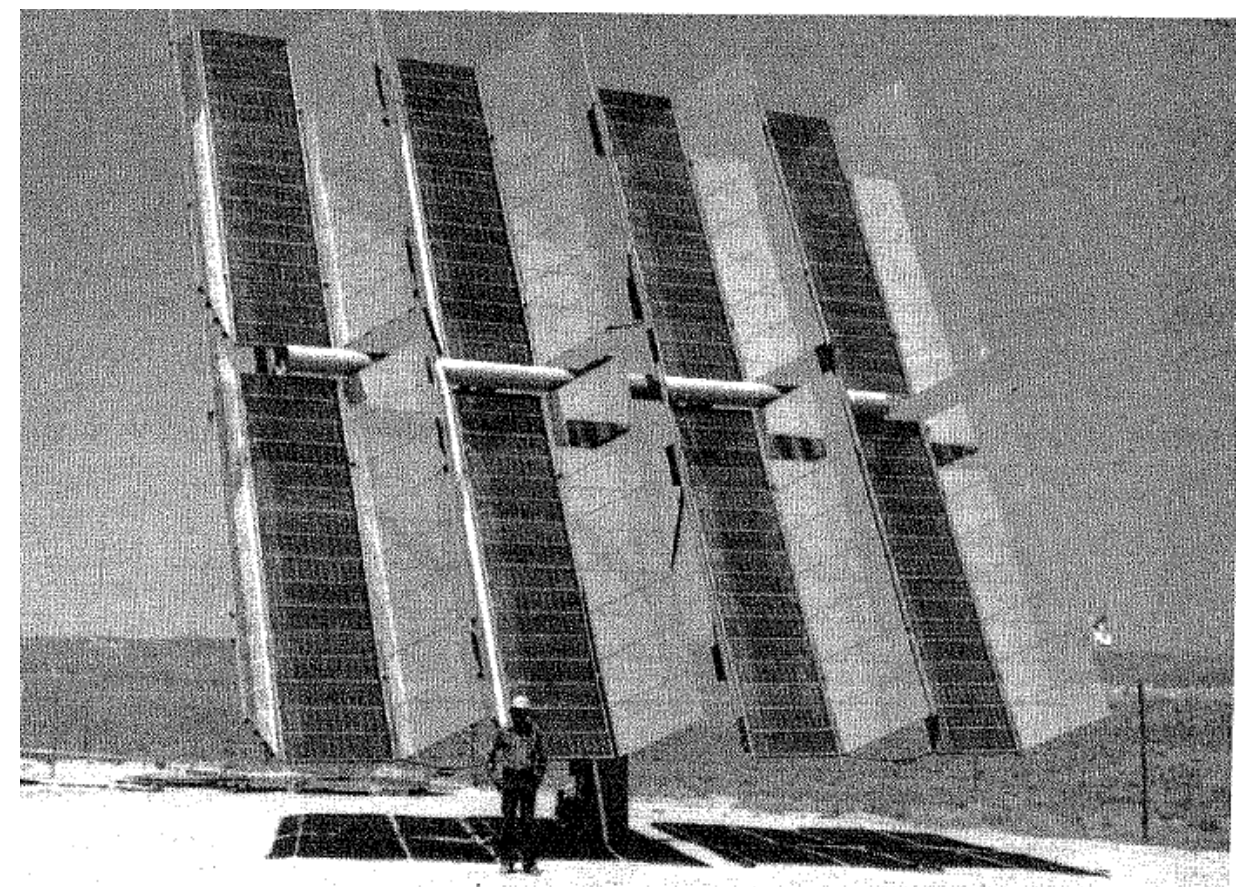

Figure 6: An image of the mirror-augmented tracker at Carrizo Plains ${ }^{19}$.

All segments utilized single-crystalline flat panel modules. It is now understood that the high annual degradation rate observed at Carrizo Plains arose from various design and fabrication errors in the PV modules. This project was expected to be an alternative energy source for our modern society. However, it failed because it exhibited power degradation rates of $10 \%$ per year, with a $40 \%$ power drop over the first four years 
$(\text { Figure } 7)^{20}$. The various modes of PV degradation exhibited linear, sub-linear or supralinear degradation rates. Initially, it was suggested that the use of solar mirror augmentation was the cause of the extreme degradation rate ${ }^{21}$. Recently, Michael Kempe's research group from DOE's National Renewable Energy Laboratory (NREL) proposed that several design errors, such as neglecting the UV absorbing glass front sheet that are now standard in all modules, poor formulation and various parameters of the EVA encapsulant and poor choice of the PV cell, could have lead to such a fast degradation rate ${ }^{22}$.

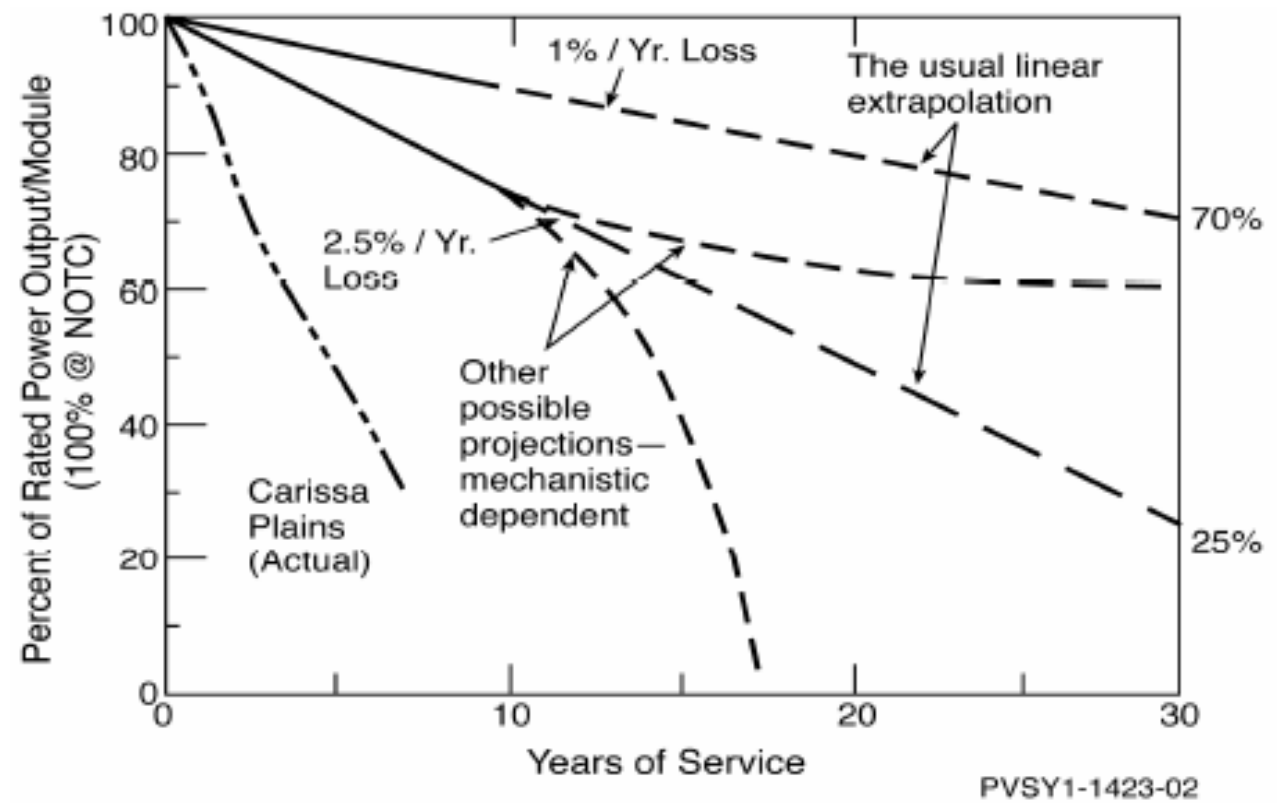

Figure 7: Power degradation of the Carrizo Plains compared to typical degradation rates for current flat-panel PV modules ${ }^{20}$.

In 1975, the National Photovoltaics Program initiated a series of PV module development activities, designated block I through V. The goal of this project was to assist industry in developing the most advanced modules by performing failure analysis 
and extensive testing. Figure 8 shows the examples of block I through block $\mathrm{V}^{23}$. The modules of block IV and V in Figure 8 are comparable to modern c-Si modules.

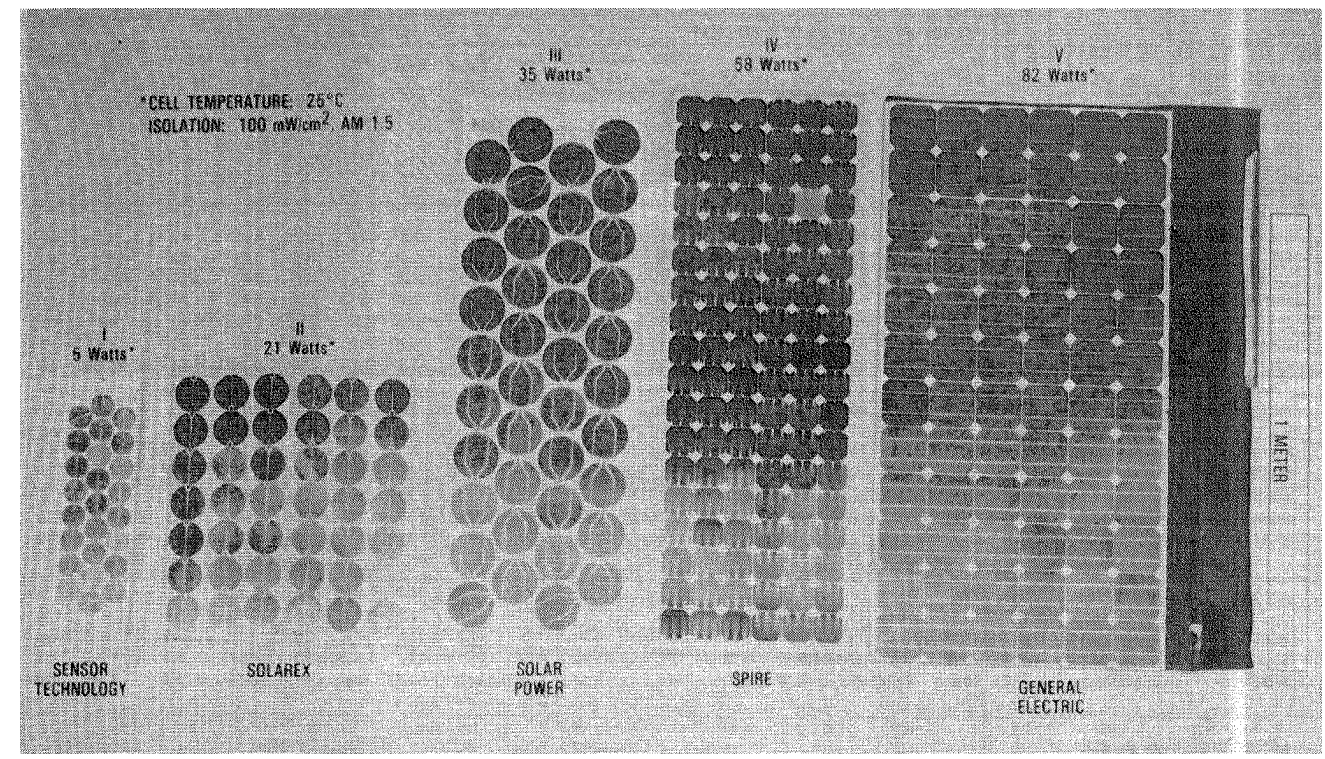

Figure 8: Representative modules of block I through block V (from left to right) ${ }^{23}$.

The representative mechanical and electrical characteristics of the modules are listed in Table 1. The cast silicone rubber encapsulant, as a top surface in block I-III offered inadequate protection against cell fracture caused by hail impact and accumulated dirt. It was concluded that block $\mathrm{V}$ modules have several common characteristics: large size, glass/ Ethylene vinyl acetate (EVA)/plastic-film configuration, high packing factor, rectangular in shape, parallel circuit strings and by-pass diodes. EVA was chosen as the encapsulant in block $\mathrm{V}$ because it provided superior protection to the circuitry and is less expensive than polyvinyl butyral (PVB). The success of block program approach was the fact that most design details have been adopted internationally. Arco Solar, Inc. was one of the companies in the block program; therefore, the PV modules used at Carrizo Plains were similar to the specifications of the block $\mathrm{V}$ modules. In the next section, possible degradation mechanisms at Carrizo Plains will be discussed. 
Table 1: Representative characteristics of block modules ${ }^{23}$.

\begin{tabular}{|c|c|c|c|c|c|}
\hline & I & II & III & IV & $\mathrm{V}$ \\
\hline Area $\left(\mathrm{m}^{2}\right)$ & 0.1 & 0.4 & 0.3 & 0.6 & 1.1 \\
\hline Weight $(\mathrm{kg})$ & 2 & 5 & 5 & 9 & 17 \\
\hline Superstrate & $\begin{array}{l}\text { Silicone } \\
\text { Rubber }\end{array}$ & $\begin{array}{l}\text { Silicone } \\
\text { Rubber }\end{array}$ & $\begin{array}{l}\text { Silicone } \\
\text { Rubber }\end{array}$ & Glass & Glass \\
\hline Substrate & Rigid Pan & Rigid Pan & Rigid Pan & $\begin{array}{c}\text { Flexible } \\
\text { Sheet }\end{array}$ & $\begin{array}{c}\text { Flexible } \\
\text { Laminate }\end{array}$ \\
\hline Encapsulation System & Cast & Cast & Cast & Laminated & Laminated \\
\hline Encapsulation Material & $\begin{array}{l}\text { Silicone } \\
\text { Rubber }\end{array}$ & $\begin{array}{l}\text { Silicone } \\
\text { Rubber }\end{array}$ & $\begin{array}{l}\text { Silicone } \\
\text { Rubber }\end{array}$ & PVB & EVA \\
\hline Cells: Quantity & 21 & 42 & 43 & 75 & 117 \\
\hline Size & Dia: 76 & Dia: 76 & Dia: 76 & $95 \times 95$ & $100 \times 100$ \\
\hline Configuration & Round & Round & Round & Shaped & Shaped \\
\hline Fault Tolerance: & & & & & \\
\hline Parallel Cell Strings & None & None & None & 3 & 6 \\
\hline Intercon. Redundancy & None & Minor & Minor & Much & Much \\
\hline By-Pass Diodes & No & No & No & Yes & Yes \\
\hline Perform. at $28^{\circ} \mathrm{C}$ : & & & & & \\
\hline Power Max. (W) & 8 & 24 & 26 & 54 & 112 \\
\hline Module Efficiency (\%) & 5.8 & 6.7 & 7.4 & 9.1 & 10.6 \\
\hline Encap. Cell Efficiency & 10.6 & 11.2 & 11.5 & 11.8 & 12.3 \\
\hline
\end{tabular}




\subsubsection{PV Module Design- Front Glass}

PV modules are exposed to extreme weather conditions that can include a combination of stressors such as heat, salty humidity, high voltage, mechanical stress, thermal cycling and UV radiation. Glass is an integral and important element in PV panels. The primary function of the front glass is to protect the module from the harsh weather. In addition, glass is necessary to add robustness to the PV module. Low-iron glass is used to increase optical transmission of light to the PV module. Cerium containing glass can block UV-B radiation, which can help reduce the degradation rate of EVA from damaging UV radiation. Figure 9 shows the EVA specimens behind non-Ce glass, which shows browning after exposure to 42 suns irradiation for 1568 hours $^{24}$. It can be concluded that the Ce glass can reduce the rate of EVA degradation. Therefore, the Ce glass is one of the solutions to help mitigate the failures seen at Carrizo Plains because the degradation was partially the result of polymer embrittlement, delamination and discoloration ${ }^{25}$.

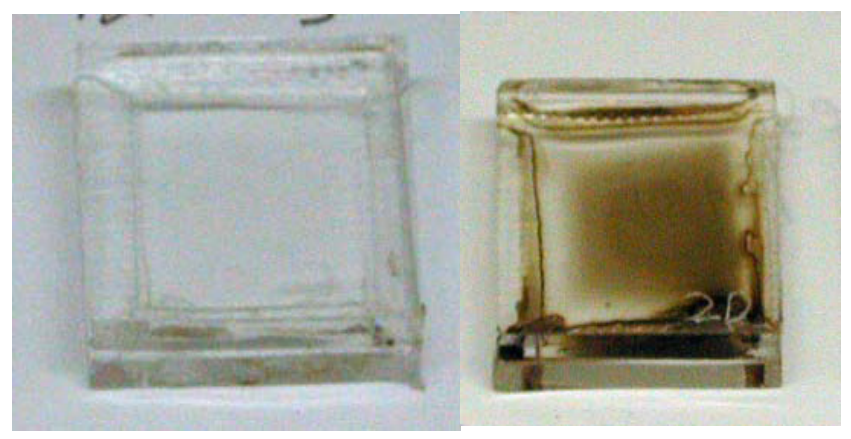

Figure 9: The discoloration of EVA specimen after exposure to 42 suns cover by Ce glass (left) and non-Ce glass (right) ${ }^{24}$. 


\subsubsection{PV Module Design- Encapsulation Materials}

In PV modules, polymeric materials provide electrical insulation and protect modules from mechanical damage and environmentally induced corrosion. The development of PV technology started in the 1950s. Polydimethyl siloxane (PDMS) base encapsulants were chosen in designs because of its exceptional UV resistance and thermal stability. In 1970-1980s, Flat Solar Array PV block program developed a lowcost alternative encapsulation films composed of copolymers of EVA as an encapsulant. However, it was found that EVA has notable problems with yellowing and browning that contributed to catastrophic failure at Carrizo Plains. In recent years, scientists have developed better stabilizer formulations for EVA to achieve low glass transition, low modulus, low crystallinity, high transmittance and convenient melting temperature $\left(45^{\circ} \mathrm{C}\right.$ $\sim 65^{\circ} \mathrm{C}$ ). Figure 10 illustrates various additives of EVA formulation. For example, $1 \%$ to $2 \%$ of thermally-activated peroxide is used for cross-linking at elevated temperature (above $140^{\circ} \mathrm{C}$ for 2 minutes). Trialkoxy silane is used to promote adhesion between EVA and inorganic surfaces for protecting inorganic surfaces from corrosion. Benzotriazole is used as UV absorber instead of benzophenone, lupersol 101 (peroxide) and phenyl phosphonite, which resulted in significant degradation of EVA at Carrizo Plains ${ }^{26}$. 

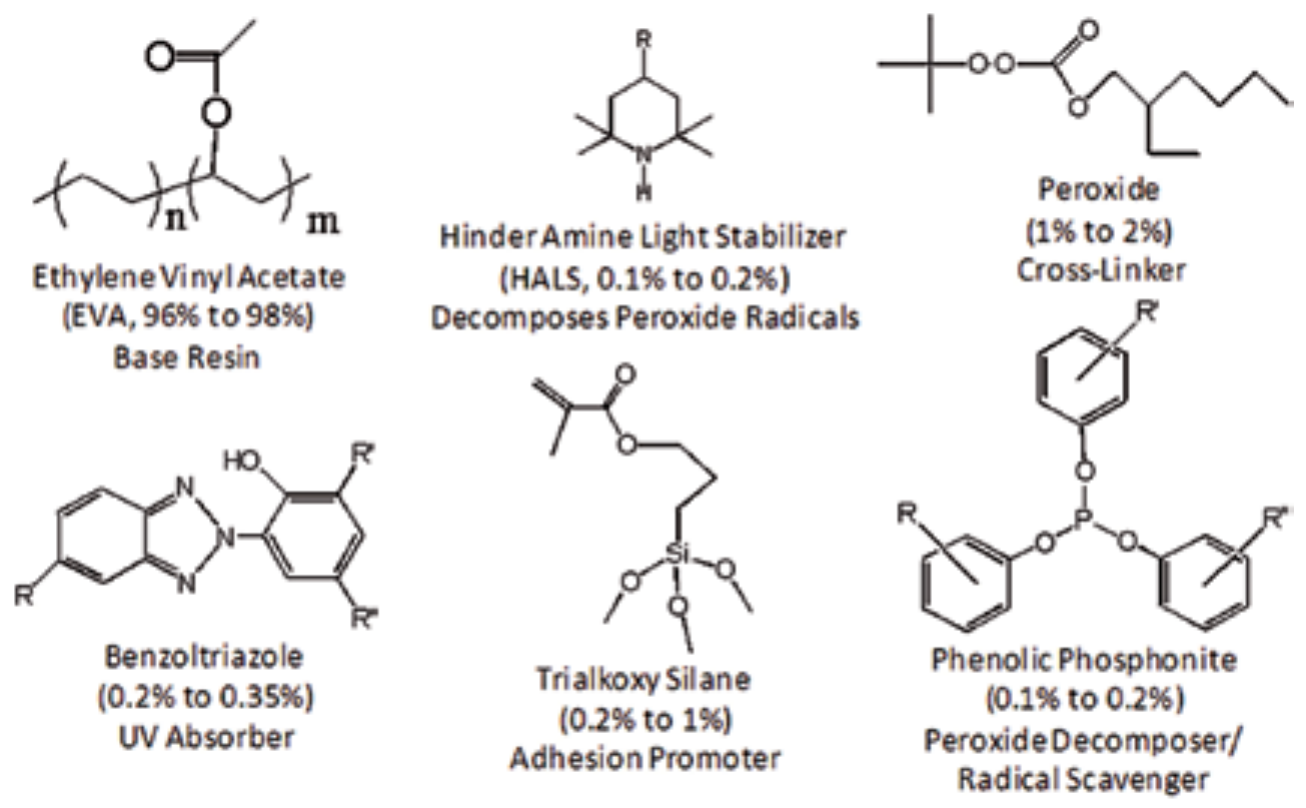

Figure 10: Example formulations of PV encapsulant ${ }^{26}$.

\subsubsection{Important Factors of Degradation Mechanisms at Carrizo Plains}

According to Dr. Kempe's suggestions, the problem of design errors, poor formulation and poor choice of the PV cell can be easily modified by renewing technology ${ }^{26}$. The properties of the encapsulant are crucial to the long-term performance of PV modules. In the case of Carrizo Plains, it was found that solder joint failure was the primary issue in the PV modules because of poor module manufacturing, which led to hotspots and failures (lack of a protection diode). Secondly, non-Ce glass allowed UV irradiant penetrating through and caused photodarkening of EVA encapsulant. Due to better formulations of EVA caused by adequate curing rates and additional additives, the encapsulants are better protected against photodegradation ${ }^{26}$. The corrosion of front surface metallization also affected the lifetime of the modules. For the long-term exposure of a large area power plant, acid and humidity are also concern. 
According to previous degradation modes mentioned above, the new analysis of the failure at Carrizo Plains showed that the primary cause of degradation was not due to the mirror-augmentation ${ }^{25}$. The primary degradation mechanism in Carrizo Plains was the solder joint failure. The non-Ce containing glass and the formulation of EVA were the second and third degradation mechanism, respectively. Mirror-augmentation was in fact a minor cause of the rapid degradation seen at Carrizo Plains.

The cost reduction is still an important research topic for the commercialization of solar energy. With an improved understanding of the primary degradation mechanisms at Carrizo Plains, the MAPV system is still a valid solution to providing a low-cost energy source. Therefore, the investigation of the increase in power production associated with fixed MAPV is worthwhile.

\section{Simulation Methods and Tools}

This section describes the simulation methods and tools used to obtain power production generated by fixed MAPV system. The 1D model calculations achieved by Dave Hollingshead at Replex Plastics ${ }^{27}$. An outdoor test facility will be introduced to compare with the indoor simulations. The sample nomenclature of fixed MAPV model will also be introduced.

\subsection{D Model for Annual Irradiance by MatLab}

Determining the power production potential for a fixed MAPV is necessary. In order to obtain maximized power output for a particular location, the mirror and module configuration via 1D optical simulation in $\mathrm{MatLab}^{28}$ needed to be optimized. For this calculation the total annual incident power on the mirror-augmented module (the MAPV 
system) at any given time will be optimized. It is noteworthy that for fixed MAPV systems, (i.e. systems that do not use trackers to orient the MAPV system with the sun) flat mirrors reflect light onto the PV panel creating discrete "bands" of illumination across the panel. The band intensity and position depends on four important parameters: sun position represented as azimuth (east/west angle) and elevation (winter/summer) angle and the mirror \& module tilt angles. The sun's position calculation for every minute for the given installation location is based on a technical report developed by Reda and Andreas in NREL ${ }^{29}$. The air mass is the path length which light takes through the atmosphere normalized to the shortest possible path length. The air mass quantifies the reduction in the power of light as it passes through the atmosphere and is absorbed by air and dust ${ }^{33}$. The theoretical air mass incorporates the curvature of the earth as given by Equation $1^{30}$.

$$
\text { AirMass }=\frac{1}{\cos \left(\alpha_{\text {sun }}\right)+0.50572\left(96.07995-\alpha_{\text {sun }}\right)}
$$

In Equation $1, \alpha_{\text {sun }}$ is the sun zenith angle ( $=90^{\circ}$ - sun elevation angle). The intensity of the direct component of sunlight throughout each day $\left(\mathrm{I}_{\text {direct }}\right)$ can be determined as a function of air mass from the experimentally determined by Equation $2^{33}$.

$$
I_{\text {direct }}=1.353 \times\left(0.7^{A M^{0.678}}\right)
$$

The theoretical irradiance calculation includes the $10 \%$ diffuse radiation of the direct component. Thus on a clear day, the global irradiance ( $\left.\mathrm{I}_{\text {global }}\right)$ on a module perpendicular to the sun's rays is determined by Equation $3^{31}$. 


$$
I_{\text {global }}=1.1 \times\left(I_{\text {direct }}\right)
$$

The power incident on a PV module depends not only on the power contained in the sunlight, but also on the angle between the module and the sun. When the absorbing surface and the sunlight are perpendicular to each other, the power density on the surface is equal to that of the sunlight (in other words, the power density will always be at its maximum when the PV module is perpendicular to the sun). However, as the angle between the sun and a fixed surface is continually changing, the power density on a fixed PV module is less than that of the incident sunlight. Therefore, for a PV module at an arbitrary tilt and orientation, Equation 4 provides the raw incident panel intensity $\left(\mathrm{S}_{\text {panel }}\right)^{32}$ :

$$
S_{\text {panel }}=I_{\text {global }}\left[\cos \left(\theta_{\text {sun }}\right) \sin \left(\theta_{\text {panel }}\right) \cos \left(\phi_{\text {panel }}-\phi_{\text {sun }}\right)+\sin \left(\theta_{\text {sun }}\right) \cos \left(\theta_{\text {panel }}\right)\right]
$$

Where, $\theta_{\text {sun }}$ is the sun elevation angle, $\phi_{\text {sun }}$ is the sun azimuth angle, $\theta_{\text {panel }}$ is the panel tilt angle and $\phi_{\text {panel }}$ is the azimuth angle that the panel faces ${ }^{33}$. A PV panel lying flat on the ground has $\theta_{\text {panel }}=0^{\circ}$ and a vertical panel has a $\theta_{\text {panel }}=90^{\circ}$. A similar concept was used to represent the incident solar mirror intensity $\left(\mathrm{S}_{\text {mirror }}\right)$, reflected onto the PV panel shown in Equation 5:

$$
S_{\text {mirror }}=I_{\text {global }}\left[\cos \left(\theta_{\text {sun }}\right) \sin \left(\theta_{\text {mirror }}\right) \cos \left(\phi_{\text {mirror }}-\phi_{\text {sun }}\right)+\sin \left(\theta_{\text {sun }}\right) \cos \left(\theta_{\text {mirror }}\right)\right] R_{\text {mirror }}
$$

where $\theta_{\text {mirror }}$ is the mirror tilt angle, $\phi_{\text {mirror }}$ is the azimuth angle that the mirror faces, and $R_{\text {mirror }}$ is the reflectivity of the solar mirror developed by Replex Plastics. By adding the light intensities of $\mathrm{PV}$ panel and reflective mirror, the total amount of power 
generated by fixed MAPV can be obtained.

Figure 11 illustrates the mirror throw distance in MAPV system. The mirror throw distance in the model determined the region, which can be augmented by solar mirror. The mirror throw distance can be obtained by simply calculating the geometry of the MAPV system as shown in equation 6.

$$
\text { Dist }_{\text {mirror }}=\frac{L_{\text {mirror }} \sin \left(\theta_{\text {sun }}-\theta_{\text {mirror }}\right)}{\sin \left(\theta_{\text {panel }}-\theta_{\text {sun }}+2 \theta_{\text {mirror }}\right)}
$$

The shadow distance can also be obtained to represent the region, which was not augmented by reflected ray due to mirror blocking as shown in equation 7 .

$$
\text { Dist }_{\text {shadow }}=\frac{L_{\text {mirror }} \sin \left(\theta_{\text {mirror }}-\theta_{\text {sun }}\right)}{\sin \left(\theta_{\text {panel }}+\theta_{\text {sun }}\right)}
$$

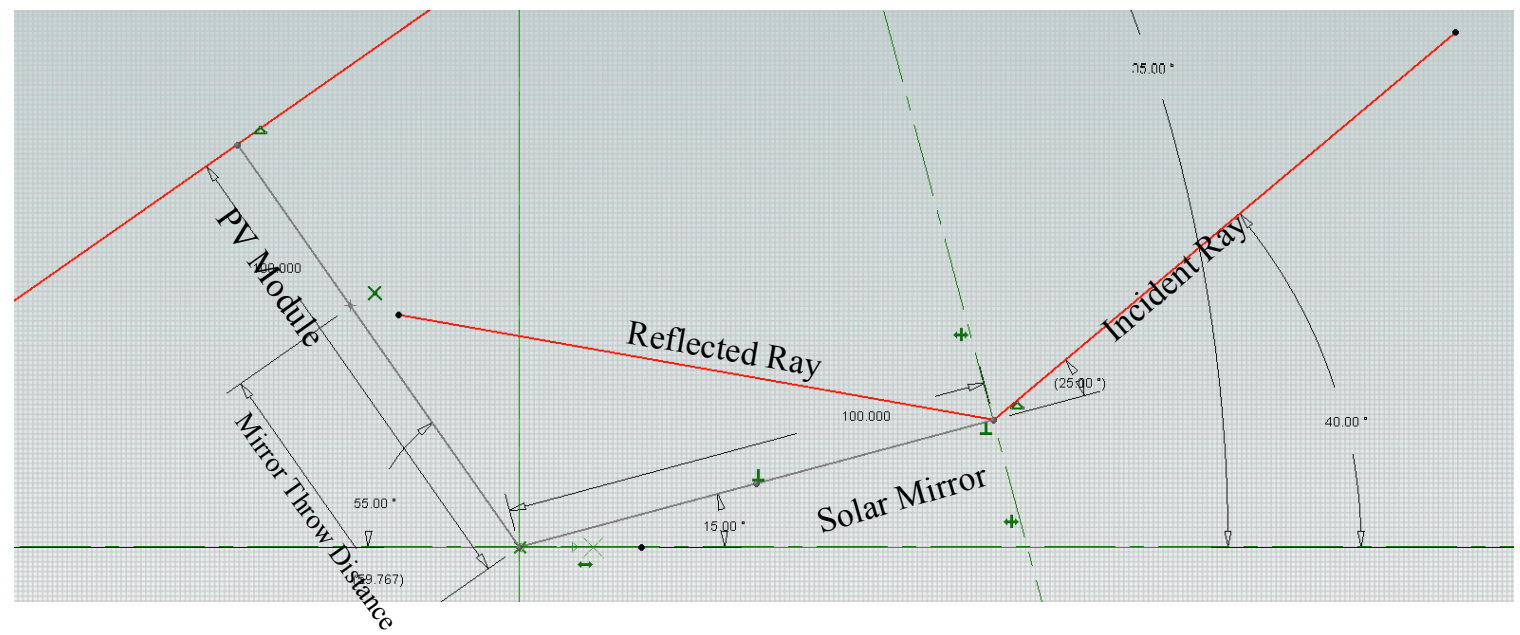

Figure 11. The sketch of mirror throw distance on MAPV architecture.

The longitude and latitude were set to Columbus, Ohio. No cloud cover or weather is considered and no end effects are included because mirrors are assumed to be infinitely 
long. The initial MatLab model started from setting a specific tilt angle for the module and mirror (e. g. panel tilt at $50^{\circ}$ and mirror tilt at $10^{\circ}$ ).

Then, the sun position for a given installation location is computed and the power density of the light (in $\mathrm{kW} / \mathrm{m}^{2}$ ) that hits the panel at a given instant in time (e.g. 7:45 am on January $1^{\text {st }}$ ) is found. The incident illumination on module and mirrors is based on sun's azimuth and elevation angle ${ }^{33}$. The illumination pattern of the light is compared with the module's string architecture and electrical output is estimated. This process is repeated for the entire year in order to calculate the cumulative amount of annual incident power. From the description above, the amount of power for a specific model design can be estimated. Further calculations can be performed for different system designs (e.g. panel tilt $=35^{\circ}$, mirror tilt $=0^{\circ}$ ) by simply repeating the above process.

\subsection{D Geometric Optical Analysis by TracePro ${ }^{\circ}$}

In addition to the 1D optical irradiance model, a full 3D model of irradiance nonuniformity is needed for MAPV optimization. The 3D optical analysis helps to observe the uniformity of a particular fixed MAPV system. Since the fixed PV modules were augmented by mirrors throughout the day, the occurrence of non-uniform irradiance distribution on modules is expected. The non-uniformity issue is an important factor to fully study the power production capabilities and to estimate power degradation rates. PV modules, which receive highly localized irradiance may experience faster module degradation rates. Therefore, ray-tracing is one of the best methods to simulate the light distribution on solar panel as a function of time of day and year ${ }^{34}$. Various ray-tracing software have been used to evaluate CPV, such as $\mathrm{FRED}^{35}{ }^{35}$ Apilux $^{{ }^{36}}$ ZEMAX $^{\mathrm{s}^{3738}}$ and TracePro $^{39}$. TracePro ${ }^{8}$ (version 7.0), developed by Lambda Research Corp ${ }^{40}$, was used in 
the present work to observe the irradiance distribution on the PV module. This software allows for the complete detailed ray-trace analysis on a model without making assumptions as to the order in which objects and surfaces will be intersected. At each intersection, individual rays can be subject to absorption, reflection, refraction, diffraction and scatter. Furthermore, TracePro ${ }^{\circledR}$ can construct 3D solid models geometrically and it is also compatible with other computer aided design (CAD) programs such as SolidWorks ${ }^{{ }^{4}}$.

\subsubsection{The Construction of 3D Model}

A PV panel was created along with solar mirror in SolidWorks ${ }^{\circledR}$ Kara A. Shell, a mechanical engineering from Replex Plastics, helped to build the prototype 3D model of MAPV (Figure 12) ${ }^{42}$. A $3 \mathrm{~mm}$ thick glass surface was designated as the top surface of a solar panel in order to simulate real conditions. The area ratio between solar panel and mirror is approximately 1:1 to insure that the reflected light will fully illuminate the solar panel. The small gap between panel and mirror was designed to prevent the module from dirt or snow accumulation as shown in Figure 12. 


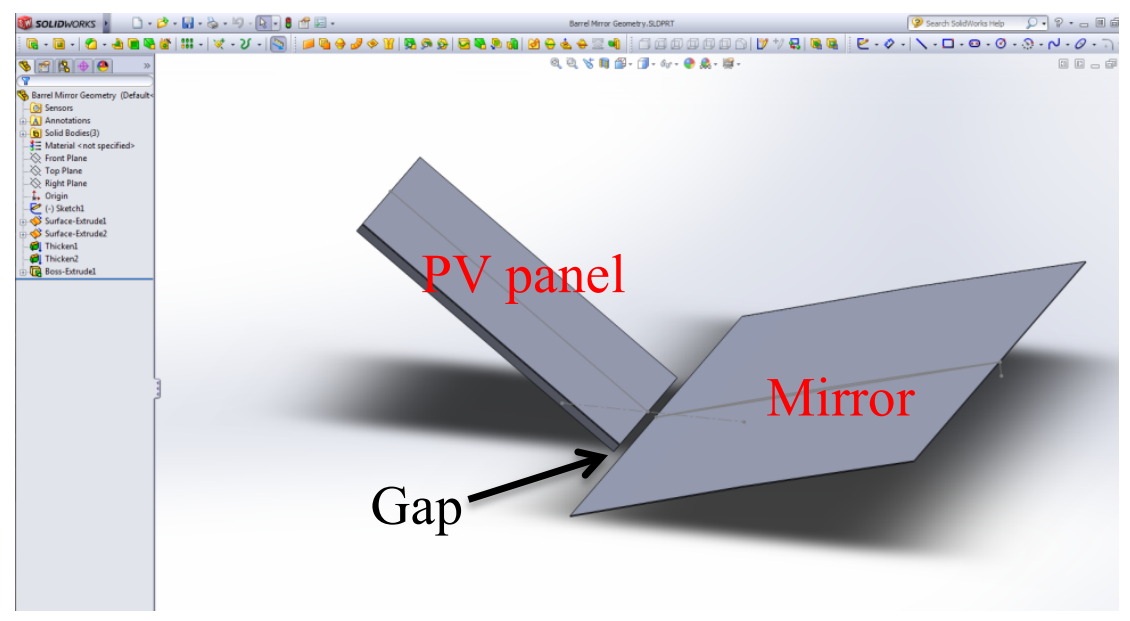

Figure 12: A 3D MAPV model constructed by commercial CAD software, SolidWorks.

A small gap was designed to prevent the module from dirt or snow accumulation.

\subsubsection{The Definition of Material and Surface Properties}

The second step is to import the designed model into TracePro ${ }^{\circ}$ and to define material and surface properties for the appropriate objects in the model as shown in Figure 13. The material and surface properties represent relevant attributes such as reflection and absorption are defined for our geometrical model. For example, the solar mirror developed by Replex Plastics, Inc. The surface property of the solar mirror was set to $88 \%$ reflectance, which allows for $88 \%$ of rays to be reflected from mirror at the selected wavelength of $546 \mathrm{~nm}$. The bulk material of the mirror was set to acrylic and the back surface material was set to aluminum. The solar panel was designed as a perfect absorber on the top surface. 


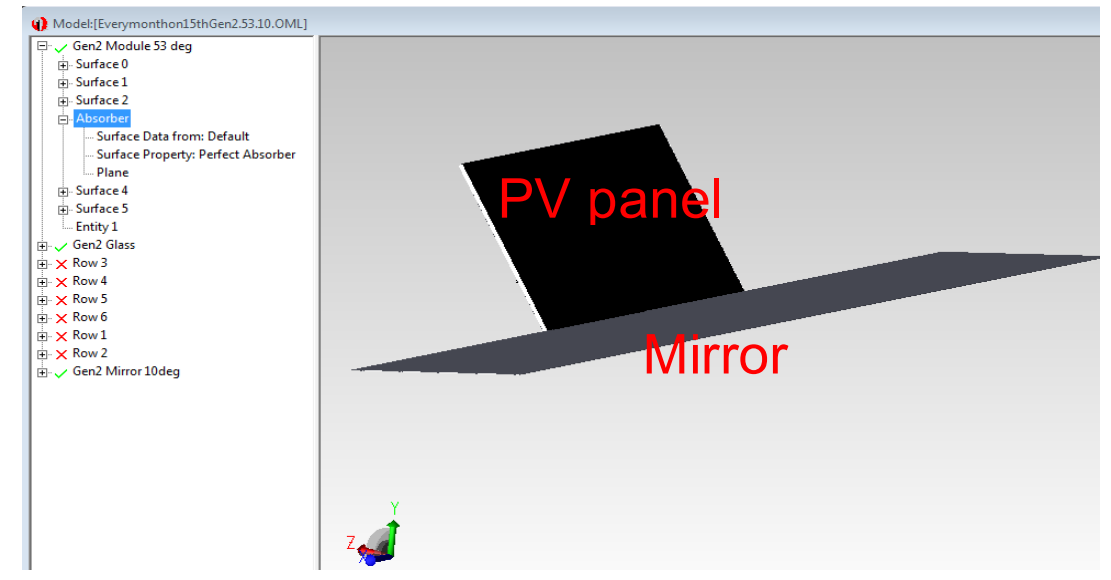

Figure 13: The 3D MAPV model was imported into TracePro ${ }^{\star}$ and surface properties were defined.

Before simulation, the light source in TracePro ${ }^{\circledR}$ was defined as grid source, which represents the sun. In a grid source type, the spatial and angular distribution of rays in a regular or random grid can be specified in the software. The number of rays to be traced and peak also can be specified. In the grid source options, rectangular was chosen as the grid boundary in order to fully illuminate the panel and mirror in the model. The grid dimensions in the model were set at $2500 \mathrm{~mm}$ in either $\mathrm{Y}$ half-height \& X-half-width. Grid pattern was set to random mode. Two million rays were used to ray trace the model with a single wavelength of $546 \mathrm{~nm}$. The unit for the flux to be emitted by the source is radiometric (Watt or $\mathrm{Watt} / \mathrm{m}^{2}$ ). Total irradiance was used as source energy. The intensity of input energy depends with the time throughout a day ${ }^{43}$. Figure 14 illustrates the MAPV model with different rectangular grid sources. The x-axis represents the east direction. These nine grid sources represent the sun from 8 a.m. through 4 p.m. Table 2 lists the sun's average intensities from 8 a.m. to 4 p.m. ${ }^{44}$. The various intensities were applied to each grid source at different times. The position setting of the grid sources were based on the azimuth and elevation angles on specific chosen date. 


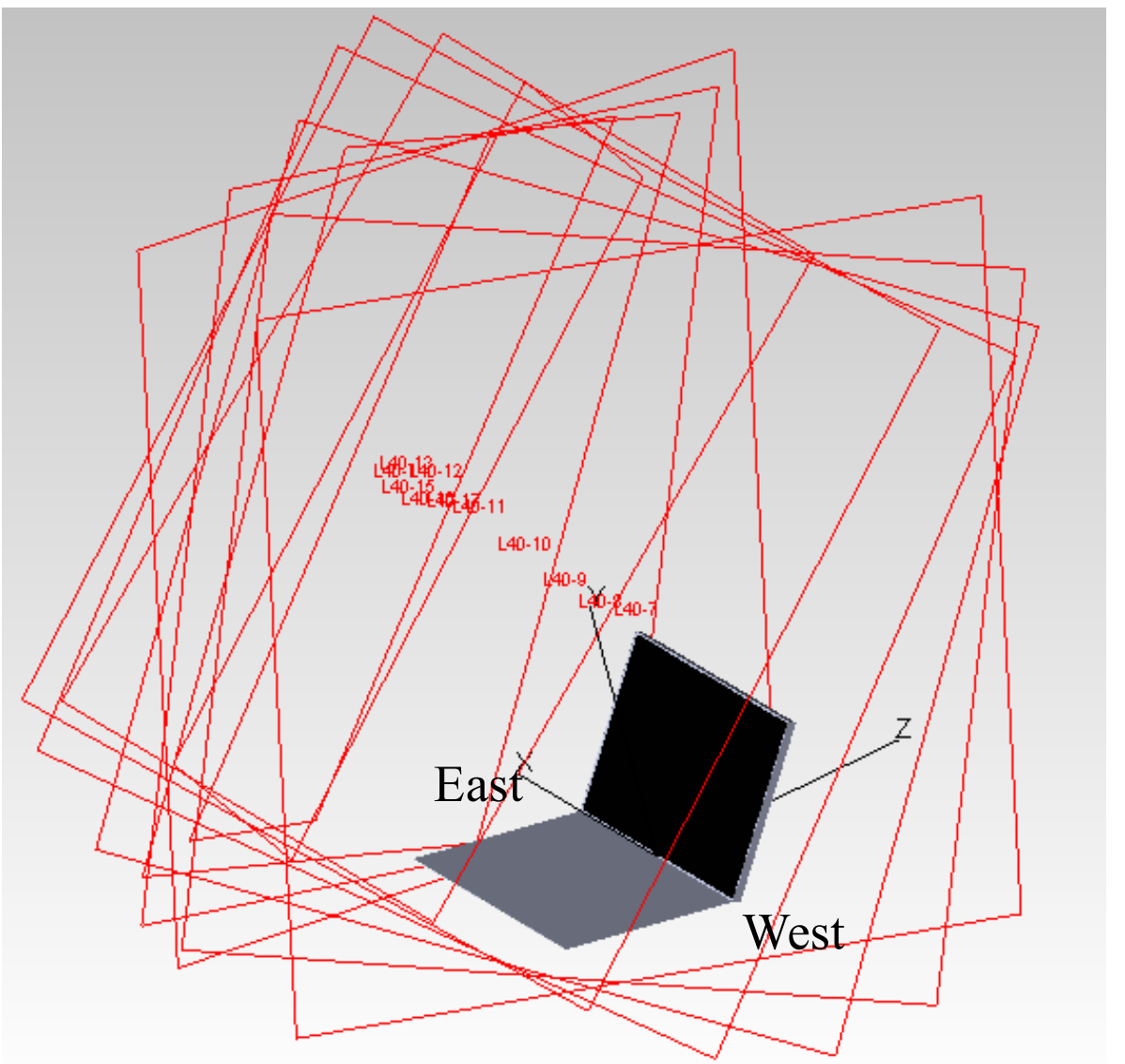

Figure 14: The MAPV model with different rectangular grid sources.

Table 2: Sun's intensities throughout a day

\begin{tabular}{|c|c|c|c|c|c|c|c|c|c|}
\hline Time & $8 \mathrm{am}$ & $9 \mathrm{am}$ & $10 \mathrm{am}$ & $11 \mathrm{am}$ & $12 \mathrm{pm}$ & $1 \mathrm{pm}$ & $2 \mathrm{pm}$ & $3 \mathrm{pm}$ & $4 \mathrm{pm}$ \\
\hline $\begin{array}{c}\text { Intensity } \\
\left(\mathrm{W} / \mathrm{m}^{2}\right)\end{array}$ & 551 & 753 & 905 & 995 & 1018 & 995 & 905 & 753 & 551 \\
\hline
\end{tabular}

\subsubsection{The Simulation Process}

The next step in the simulation process is to trace the rays from the grid light source onto the defined model. Two million rays were chosen for a reasonable ray-trace time (approximately one minute) and providing a high resolution irradiance map. The final step was to analyze the ray-trace results to determine the location, extent and distribution 
of the resultant illumination on the panel and mirrors. Figure 15 shows the ray-trace result, which contains irradiance maps and the line profile in two directions. From Figure 15a, the irradiance map shows the light distribution and average irradiance value. Figure 15b illustrates the irradiance intensity line profile from the cross in Figure 15a. The blue line and green line in Figure 15b represent the horizontal line and the vertical line in

\section{Figure 15a.}
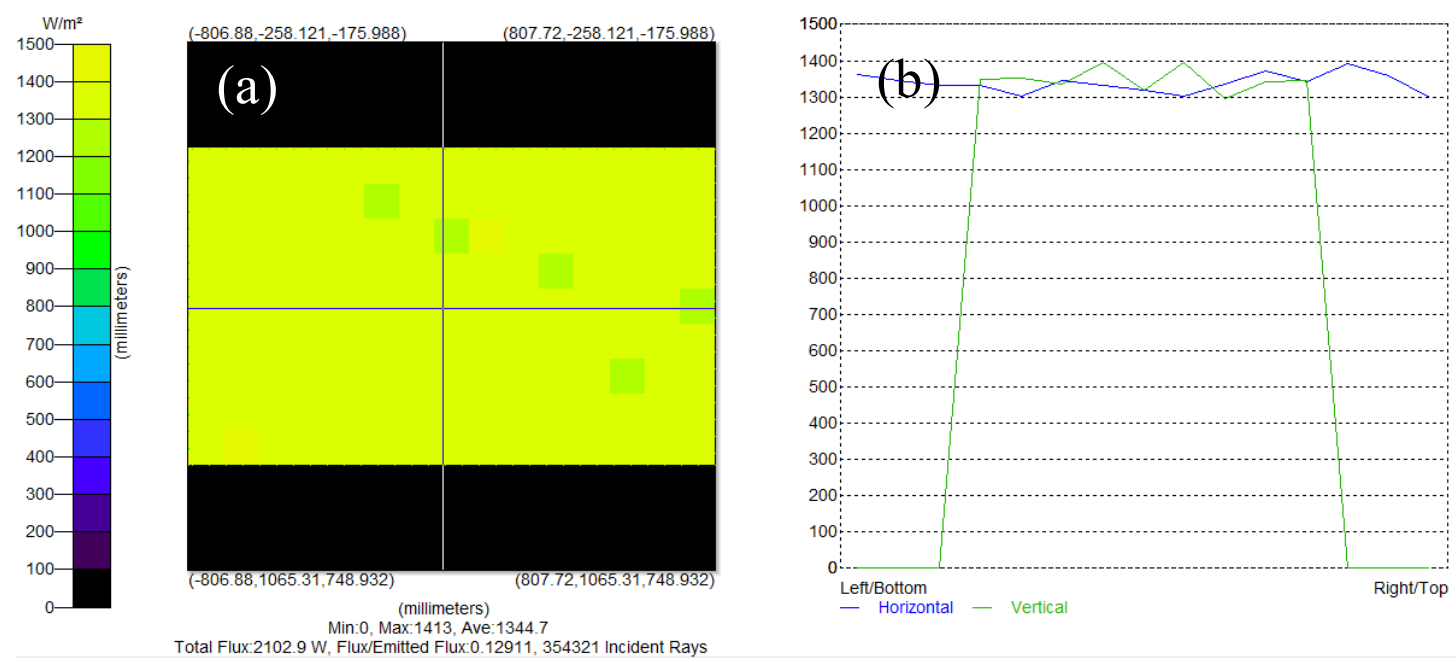

Figure 15: The ray-trace result from TracePro ${ }^{\circledR}$, which contains (a) light distribution on PV panel and (b) line profile.

\subsection{Outdoor Experimental Data Confirm with Model Simulation}

Finally, an outdoor SunFarm test facility has been constructed at Replex Plastics and SDLE Center at Case Western Reserve University for real-world data collection. A row of three consecutive PV panels (Canadian Solar CSI CS-6P) was coupled with Replex Plastics' back surface acrylic solar mirrors. A second row of non-augmented modules was also installed to enable simultaneous side-by-side comparison between augmented and non-augmented modules. An adjustable PV module mounting system developed by 
Replex Plastics was used to simulate the relative position between the sun and the fixed MAPV system by appropriately tilting module and mirror angles to achieve the correct relative positioning. This adjustable equipment can simulate the power production throughout a year within a short period of time and is called the "time machine". Using knowledge of the current sun's position, the "time machine" system was adjusted to simulate different sun positions for different times of the year. A trend of annual power output can be obtained relatively quickly. For the current-voltage (I-V) curve investigation, the I-V curves are collected from a $220 \mathrm{~W}$ Canadian Solar Inc. (CSI) CS$6 \mathrm{P}$ module ${ }^{45}$ using a Daystar Multi-Tracer ${ }^{46}$. This device is a self-contained load and data acquisition device for collecting PV module performance. The multi-tracer also collects input from auxiliary devices measuring irradiance and temperature, which can be used to normalize power measurements and I-V curves to standard temperature and conditions (STC). Contact thermocouples and solar pyranometers ${ }^{47}$ were obtained and calibrated for this purpose.

\subsection{Determination of the Date at Outdoor Field-Test Site}

The $15^{\text {th }}$ day of every month was selected as a representative day in the $3 \mathrm{D}$ optical analysis. However, some other dates were also used to compare the real-world results from the field-test site due to weather conditions at Replex Plastics. For example, January $10^{\text {th }}, 2012$ and March $10^{\text {th }}, 2012$ were chosen to observe the Gen3 model and the Gen4 model, respectively. It was found that the ray-tracing results between January $10^{\text {th }}$ and $15^{\text {th }}, 2012$ had no significant differences; therefore, the results are comparable. Similar results can be observed between March $10^{\text {th }}$ and $15^{\text {th }}, 2012$. 


\subsection{Sample Nomenclature of Fixed MAPV Model}

In this section, the method of sample nomenclature will be introduced for the numerous model designs and different model configurations. The model will be identified as different generations. For example, the PV module with no mirror was classified as Gen0. The fixed MAPV system designed by Dovetail Solar and Wind ${ }^{48}$ was classified- as Gen1, but the Gen1 model will not be discussed in this thesis. The MAPV prototype shown in Figures 12-14 is designated as Gen2 model. The Gen 2 model contains one PV module coupled with one solar mirror. The Gen 3 model was designed with a barrel mirror and the Gen 4 model with a concave mirror (Figures 37-41). After designating the generation of each model, the module and mirror tilt angles are included after the generation type in order to distinguish between model configurations. The first number following the generation type is module tilt angle, which is separated by a period. Then, the next number after the module tilt angle is the mirror tilt angle. For example, the Gen2.50.10 model is a mirror-augmented PV system with module tilt angle of $50^{\circ}$ and a mirror tilt angle of $10^{\circ}$ (Figure 17). In addition, different mirror designs induce various radius of curvature for the model. The number after the mirror tilt angle designates the degree of curvature of the mirror. For example, Gen3.55.0.90 describes a barrel mirroraugmented PV system with a module tilt angle of $55^{\circ}$, mirror tilt angle of $0^{\circ}$ and 90 inches radius of curvature for the mirror (Figure 37). Table 3 lists the model configurations, which have been studied in this thesis and its experimental tools.

Table 3. The studied model configurations and its experimental tools

\begin{tabular}{|c|c|c|c|c|}
\hline Generation & $\begin{array}{c}\text { Model } \\
\text { Configurations }\end{array}$ & $\begin{array}{c}\text { Module/Mirror } \\
\text { tilt angle }\end{array}$ & $\begin{array}{c}\text { Radius of } \\
\text { curvature }\end{array}$ & $\begin{array}{c}\text { Experimental } \\
\text { Tools }\end{array}$ \\
\hline
\end{tabular}




\begin{tabular}{|c|c|c|c|c|}
\hline 0 & Gen0.34, Gen0.55 & $\begin{array}{l}\text { Module at } 34^{\circ} \\
\text { and } 55^{\circ}\end{array}$ & None & Field-Test \\
\hline 2 & $\begin{array}{c}\text { Gen2.55.0 } \\
\text { Gen2.50.10 } \\
\text { Gen2.55.-5 35 ( } 9 \\
\text { configurations) }\end{array}$ & $\begin{array}{c}55^{\circ} / 0^{\circ} \\
50^{\circ} / 10^{\circ} \\
55^{\circ} / 5^{\circ} \sim 35^{\circ}\end{array}$ & $\begin{array}{l}\text { None } \\
\text { None } \\
\text { None }\end{array}$ & $\begin{array}{c}\text { Field-Test } \\
\text { TracePro, Time } \\
\text { Machine } \\
\text { TracePro }\end{array}$ \\
\hline 3 & $\begin{array}{c}\text { Gen3.55.0.90, } \\
\text { Gen3.55.0.540 }\end{array}$ & $55^{\circ} / 0^{\circ}$ & $\begin{array}{l}90^{\circ} \text { and } \\
540^{\circ}\end{array}$ & TracePro \\
\hline 4 & $\begin{array}{l}\text { Gen4.55.10.100, } \\
\text { Gen4.55.10.900 }\end{array}$ & $55^{\circ} / 10^{\circ}$ & $\begin{array}{c}100^{\circ} \text { and } \\
900^{\circ}\end{array}$ & TracePro \\
\hline
\end{tabular}

\section{Results}

This section shows the results of model simulations and outdoor field-test data.

\subsection{Model Configuration for Yearly Irradiance Output}

The model configuration should be determined prior to optical analysis and outdoor field tests. The 1D optical irradiance model was used to estimate the illumination pattern on a mirror-augmented module and to optimize the mirror and module configurations for maximized annual power output.

Figure 16 shows the yearly irradiance result versus mirror and module tilt angle for possible system combinations. The fixed MAPV system receives maximum irradiance (the climax of the green line) when the module tilt angle is $50^{\circ}$ and the mirror tilt angle is $10^{\circ}$ from the horizontal. The maximum estimated useful irradiance on the module obtained from this best configuration is $2.42 \mathrm{MWh}$ compared to $2.16 \mathrm{MWh}$ for PV with no mirror (dash blue curve in Fig 16). As a result, the 1D optical irradiance calculation 
estimates a $12 \%$ boost from fixed MAPV in yearly power production compared to a nonaugmented module. The best module tilt angle of non-augmented PV is $34^{\circ}$. This model configuration will be used as a baseline system to compare the power output generated from the fixed MAPV system at field-test site. In the next section, the 3D models will be constructed and analyzed based on the 1D optimum calculation results.

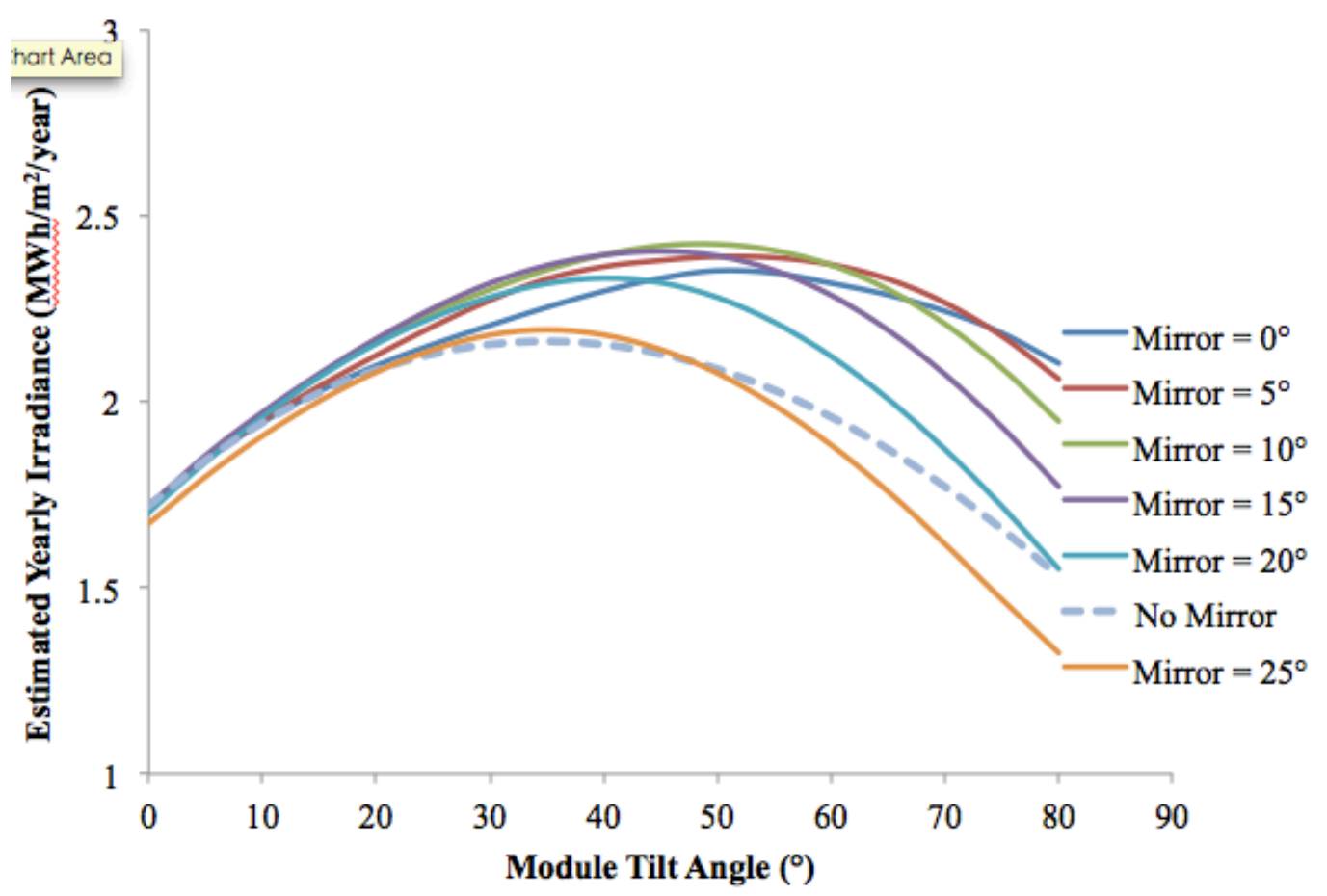

Figure 16: Estimated yearly irradiance versus module tilt angle for a number of mirror tilt angles.

\subsection{Types of Irradiance Non-Uniformity for Fixed MAPV Models}

The optical analysis starts with the creation of a solid CAD model representing the configuration designed in the previous section. Figure 17 illustrates the Gen2.50.10 model where the mirror and the module are the same length. The z-axis represents the 
northern direction. The module and mirror tilt angles based on the previous calculated results are $50^{\circ}$ and $10^{\circ}$, respectively.

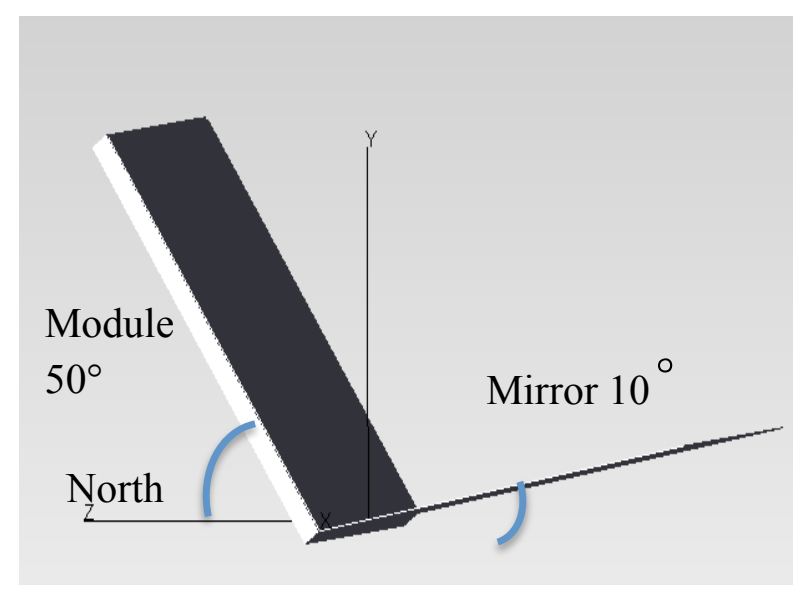

Figure 17: A 3D geometric model created by a commercial CAD software, SolidWorks.

The PV module tilt angle is $50^{\circ}$ and the mirror tilt angle is $10^{\circ}$ (Gen2.50.10).

The first grid source set up in the model simulated the sun's azimuth and elevation angles at 8 a.m. on January $10^{\text {th }}, 2012$. The irradiance intensity of the early morning sun was $551 \mathrm{~W} / \mathrm{m}^{2}$. After completing the simulation, Figure 18a shows ray-trace result and its correlated irradiance map (Figure 18b). From the ray-tracing result, it is observed that the rectangular grid source emits rays toward the 3D MAPV module. The ray-tracing result also shows the direction of the reflected rays. The irradiance map shows the irradiance intensity and its distribution (Figure 18b). The gradient scale bar on the left represents the different intensities of the irradiance distributed on the panel. The area confined by the red dashed lines represents the correlation irradiance map of the PV panel. It can be observed that a non-uniformity issue exists in the upper right region. This nonuniform region receives none or a small amount of irradiance $\left(0-100 \mathrm{~W} / \mathrm{m}^{2}\right)$. The average irradiance value on the panel was $193 \mathrm{~W} / \mathrm{m}^{2}$. 

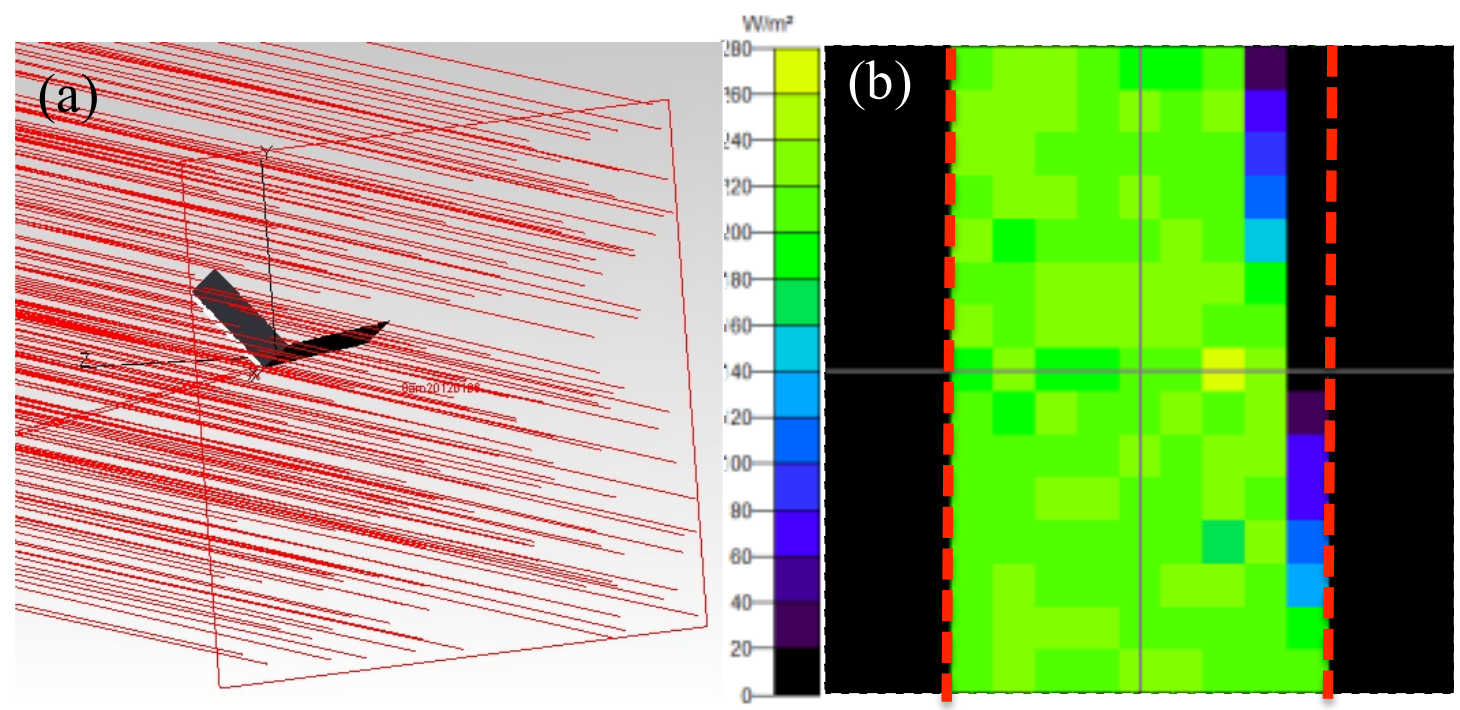

Figure 18: The ray-trace result of Gen2.50.10 model (a) and the irradiance map from model for the sun's position at 8 a.m. (b).

After the simulation at $8 \mathrm{am}$, a series of simulations were run with similar method from 9 a.m. to 4 p.m. on the same day. In these simulations, the sun's position and intensity are the only two parameters that are varied. The irradiance map for the 9 a.m. panel (Figure 19a) shows uniform irradiance distribution. The majority of the irradiance values are above $400 \mathrm{~W} / \mathrm{m}^{2}$ (green), while only a few pixels have irradiance value below $400 \mathrm{~W} / \mathrm{m}^{2}$ (light blue). The average irradiance value for the module at 9 a.m. is $427 \mathrm{~W} / \mathrm{m}^{2}$. The irradiance map at 10 a.m. (Figure 19b) shows the PV panel was augmented by mirror (green area). The highest value on single pixel is $1205 \mathrm{~W} / \mathrm{m}^{2}$. The average irradiance value for the panel at $10 \mathrm{a} . \mathrm{m}$. is $723 \mathrm{~W} / \mathrm{m}^{2}$. The green region in the $10 \mathrm{a} . \mathrm{m}$. panel shows that the area was augmented by mirror. However, because the mirroraugmented area does not reflect light back on the entire module, non-uniformity of the illumination on the module becomes a problem (marked by red circle). 

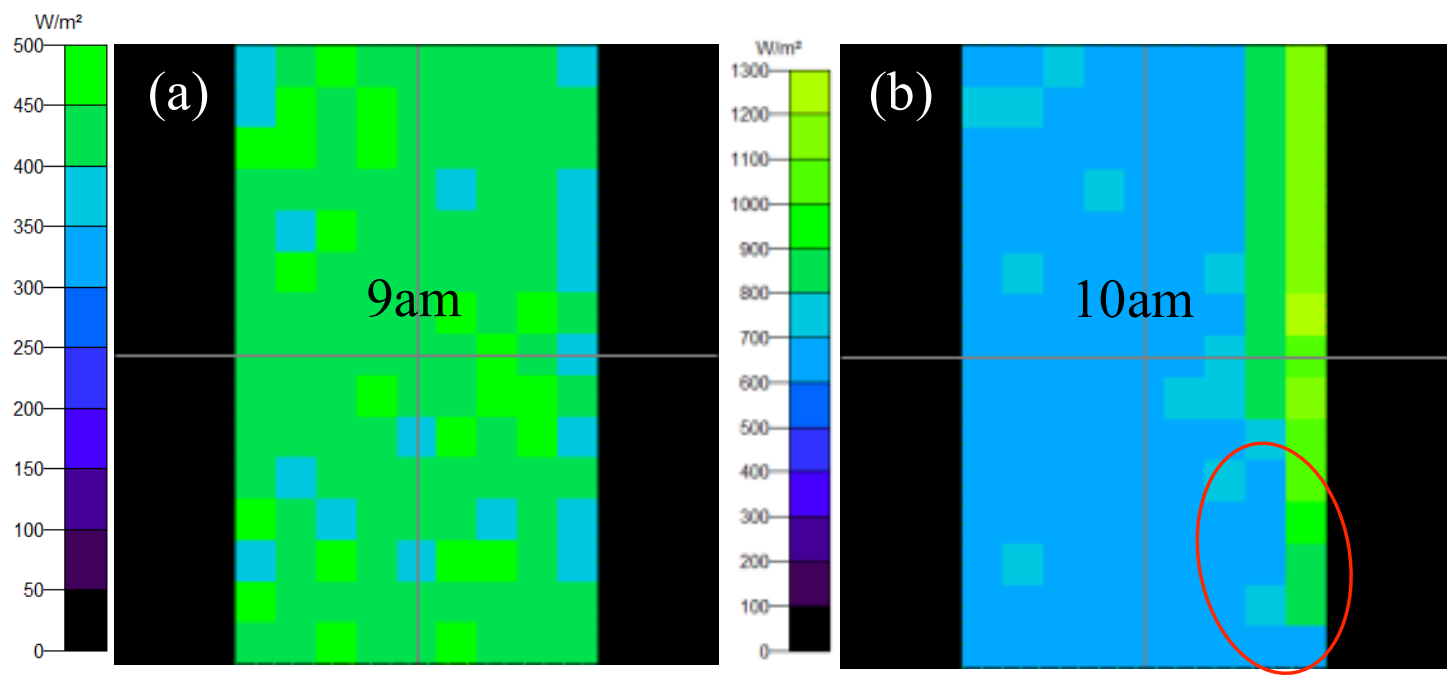

Figure 19: The irradiance maps of Gen2.50.10 model at 9 a.m. (a) and 10 a.m. model (b).

Figure 20 shows the irradiance maps at 11 a.m. (a) and 12 p.m. (b). It can be observed that the mirror-augmented region becomes larger and reaches maximum area at 12 p.m.. The average irradiance values for the models at 11 a.m. and 12 p.m. are 1017 $\mathrm{W} / \mathrm{m}^{2}$ and $1098 \mathrm{~W} / \mathrm{m}^{2}$, respectively. The non-uniform illumination can be seen in the bottom right corners of each irradiance map marked by a red circle (Figure 20).
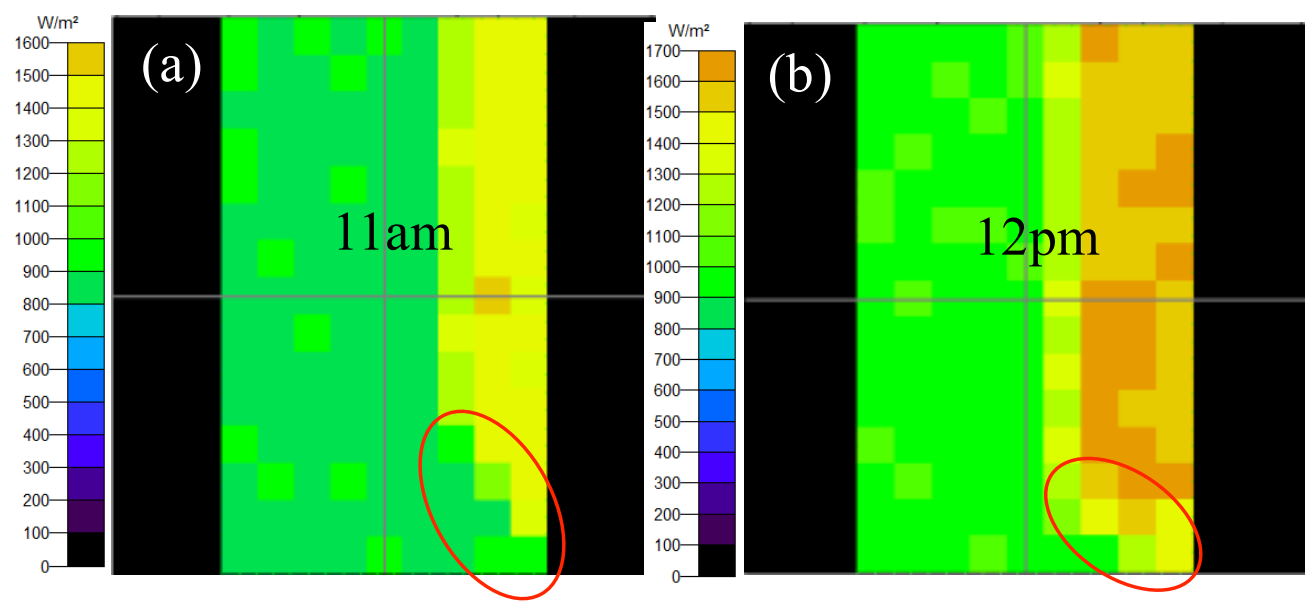

Figure 20: The irradiance maps of Gen2.50.10 model at 11 a.m. (a) and 12 p.m. (b).

Figure 21 shows the irradiance maps of panels at 1 p.m. (a) and 2 p.m. (b). The average irradiance value for the 1 p.m. and 2 p.m. panels are $1177 \mathrm{~W} / \mathrm{m}^{2}$ and $938 \mathrm{~W} / \mathrm{m}^{2}$, 
respectively. The mirror-augmented effect gradually decreased. The non-uniformity position of these two panels occurs at the upper-right side of the map, which is opposite to the irradiance non-uniformity seen for the panels in the morning (Figure 20).

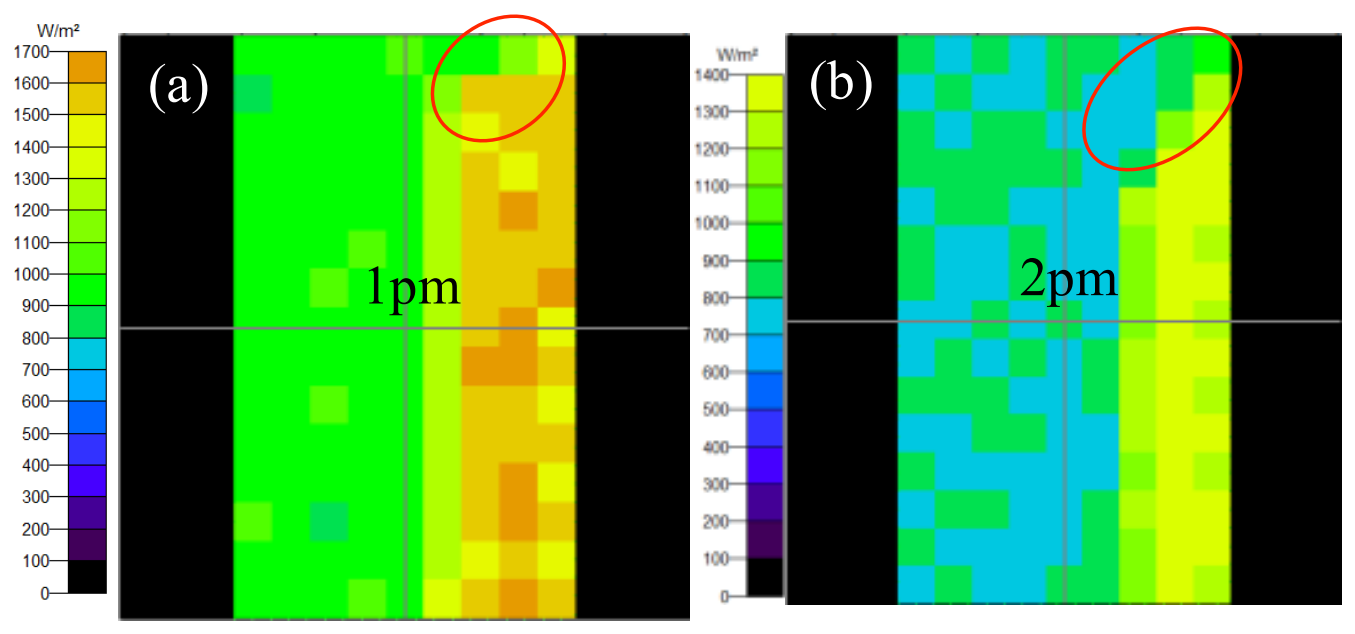

Figure 21: The irradiance maps of Gen2.50.10 model at 1 p.m. (a) and 2 p.m. (b).

Figure 22 shows the irradiance map of panels at 3 p.m. (a) and 4 p.m. (b). The average irradiance value of 3 p.m. and 4 p.m. panel are $615 \mathrm{~W} / \mathrm{m}^{2}$ and $321 \mathrm{~W} / \mathrm{m}^{2}$, respectively. The effect of mirror-augmentation is weak on the 3 p.m. panel so the green region in irradiance map is small. The non-uniformity also occurs at the upper-right corner. The mirror-augmented effect ends in the 4 p.m. panel, so the whole panel shows uniform color on the irradiance map. 

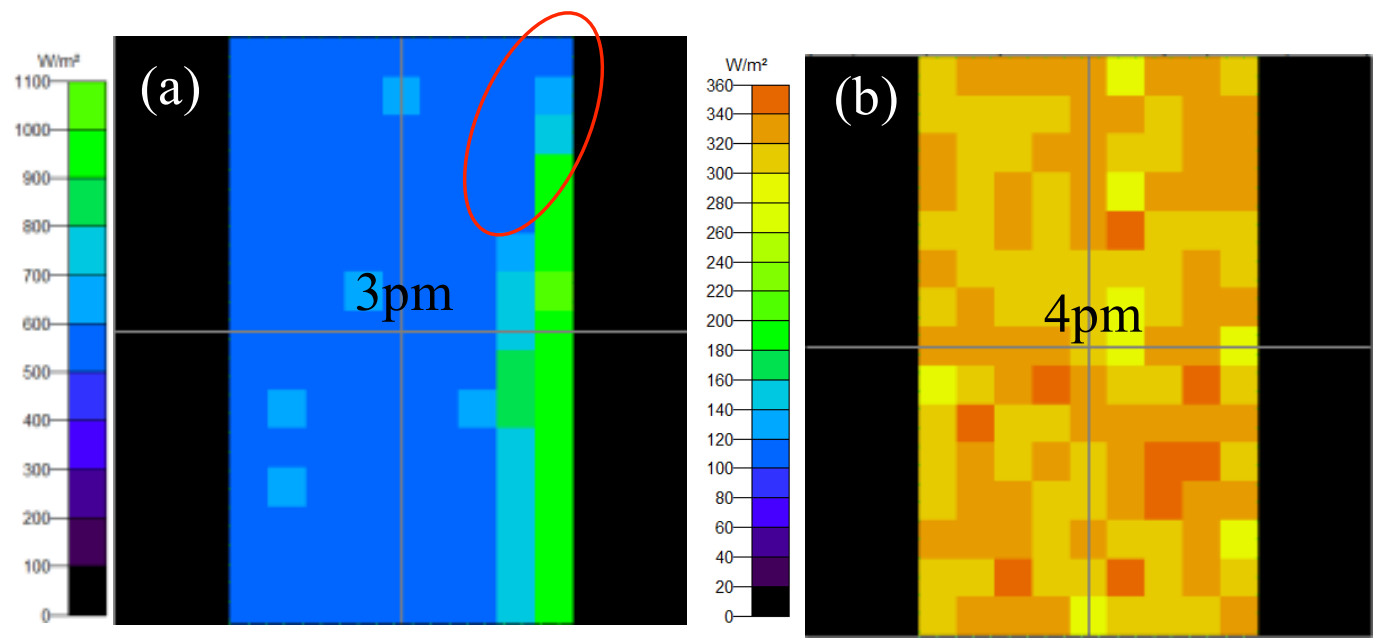

Figure 22: The irradiance maps of Gen2.50.10 model at 3 p.m. (a) and 4 p.m. (b).

Figure 23 shows the combination of irradiance maps throughout a day. It is marked by red circle that the non-uniformity issue exists on the panel at 8 a.m., 10 a.m., 11 a.m., 12 p.m., 1 p.m., 2 p.m. and 3 p.m. The non-uniform region on 8 a.m. panel caused by mirror shadowing in the early morning; therefore, it is called a "shadow effect". The lower irradiance value region on the upper right side of 8 a.m. map represents the bottom region on the west side of panel as shown in Figure 24. From 10 a.m. through 3 p.m., a non-uniformity illumination pattern exists at either bottom-right or upper-right corner of mirror-augmented region of the panel. When non-uniformity occurs only at the end of mirror-augmented area, it is called an "end effect". 


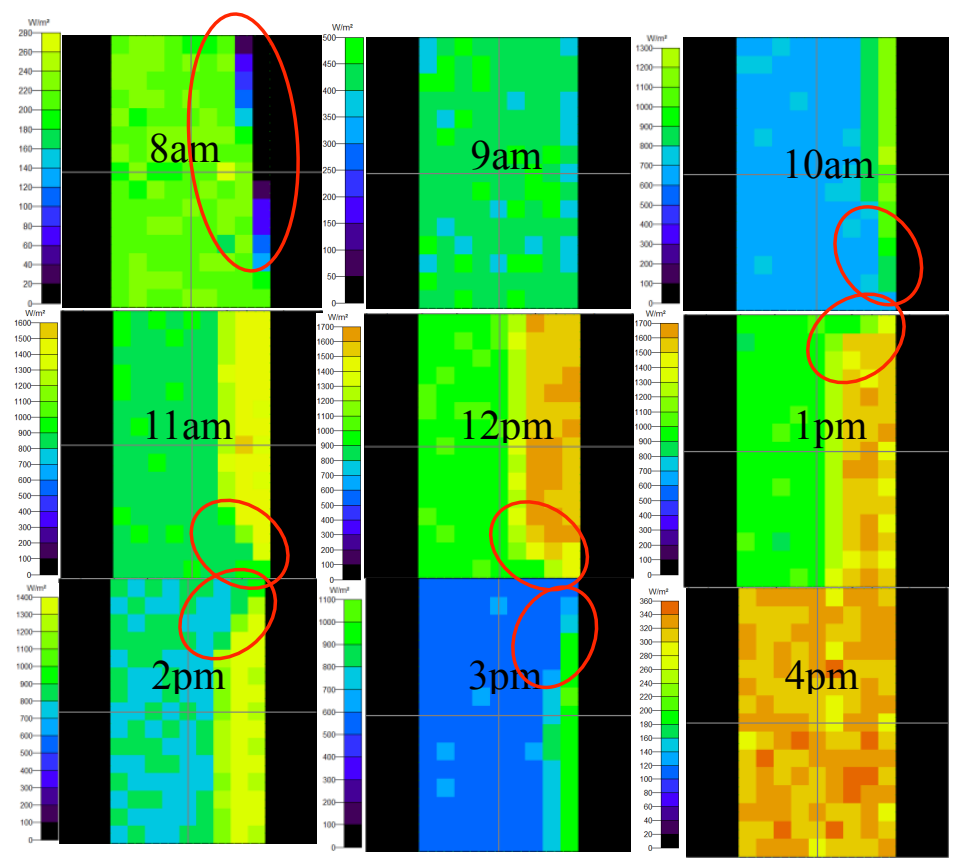

Figure 23: The light distribution on a module (Gen2.50.10) throughout a day from 8 a.m. to 4 p.m. based on the simulations.

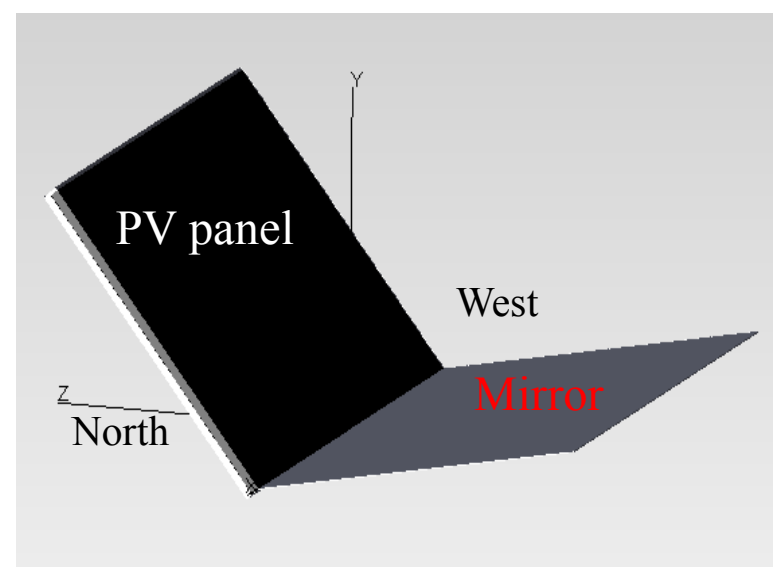

Figure 24: A 3D geometric model (Gen2.50.10) in optical analysis, where z-axis represents north, $\mathrm{x}$-axis represents east/west direction.

In order to investigate how the "end effect" occurs, tracking the pathway of light is needed. Figure 25 illustrates the ray-trace result for the 3D geometric model (Gen2.50.10) at $12 \mathrm{pm}$. The reflected light (blue rays) cannot fully illuminate the entire PV panel along 
the $\mathrm{x}$-axis direction due to the short mirror length. A deep blue circle marks the area where there are little to no reflected rays. The deep blue circle refers to the red circle of the inset irradiance map (Figure 25). Therefore, a new design MAPV model was created to solve the non-uniformity illumination pattern caused by the end effect as shown in Figure 26, which demonstrates the MAPV model with a $3 \mathrm{X}$ wide mirror. The $\mathrm{x}$-axis represents the east-west direction.

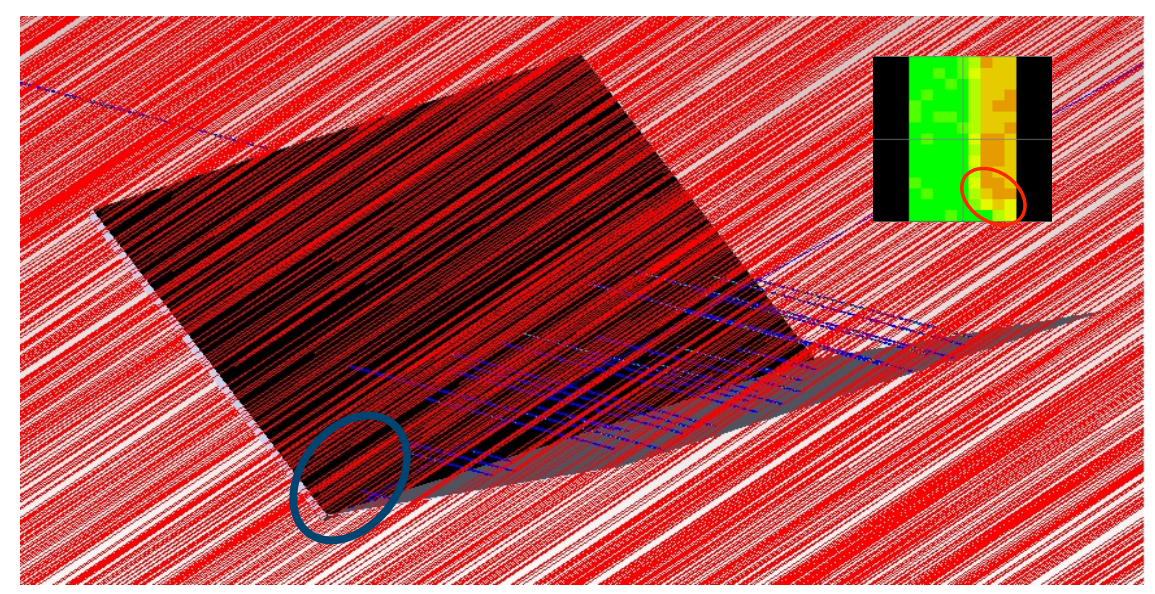

Figure 25: Ray-trace result from 12 p.m. panel, the red line represents incident rays and the blue line represents the reflected rays.

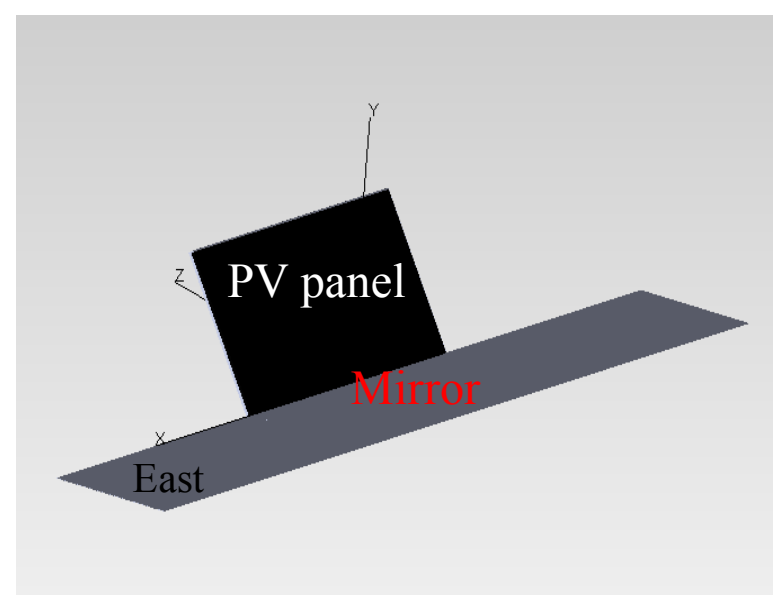

Figure 26: A 3D geometric model with $3 \mathrm{X}$ wide mirror, the $\mathrm{x}$-axis represents the eastwest direction. 
Figure 27 shows the irradiance maps of new design model through one day. It can be observed that the mirror-augmented regions have no end effect with the $3 \mathrm{X}$ wide mirror model design. From 10 a.m. to 3 p.m., the mirror-augmented regions became more uniform compared to the Gen2.50.10 model with the short mirror (Figure 23). The performance of average irradiance value on each panel had no significant change compared to the short mirror model. The 8 a.m. panel had a severe shadow effect. In the next section, a data confirmation between 3D optical analysis and field-test is presented.

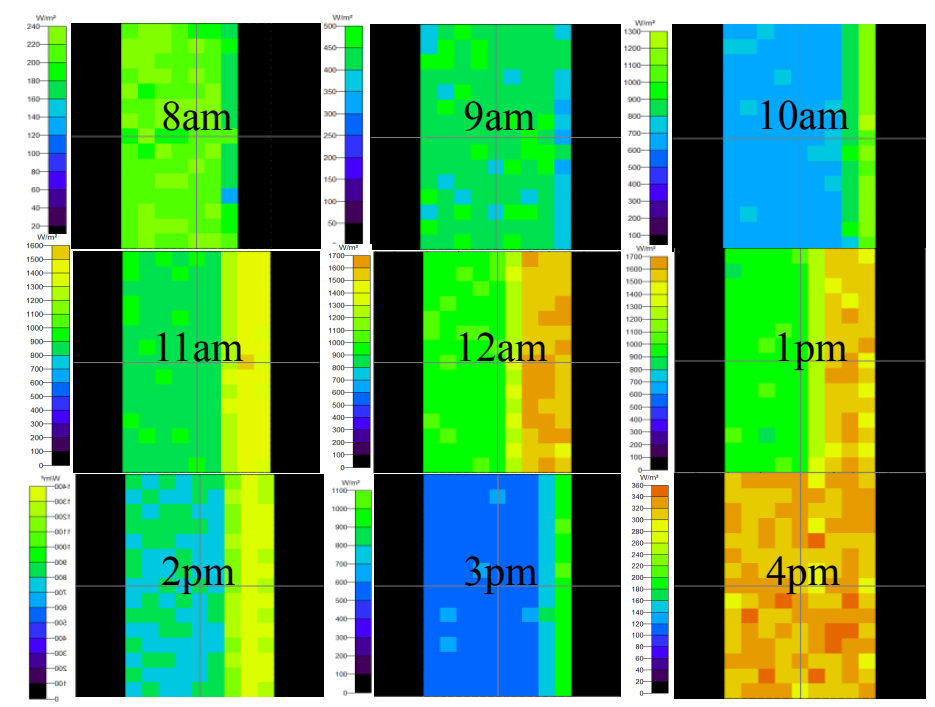

Figure 27: The irradiance maps of the $3 \mathrm{x}$ wide mirror model through one day from 8 a.m.

$$
\text { to } 4 \text { p.m. }
$$

\subsection{Comparison of 3D Models and Field-Test Data}

In addition to light distribution, the 3D optical analysis provides average irradiance values for the PV panel. Figure 28 shows the average irradiance throughout a day. The irradiance value can be increased by mirror augmentation up to $20 \%$ at noon (12 p.m.). The irradiance values for Gen2.50.10 model with a 3X wide mirror are slightly higher than the Gen2.50.10 model with short mirror. 


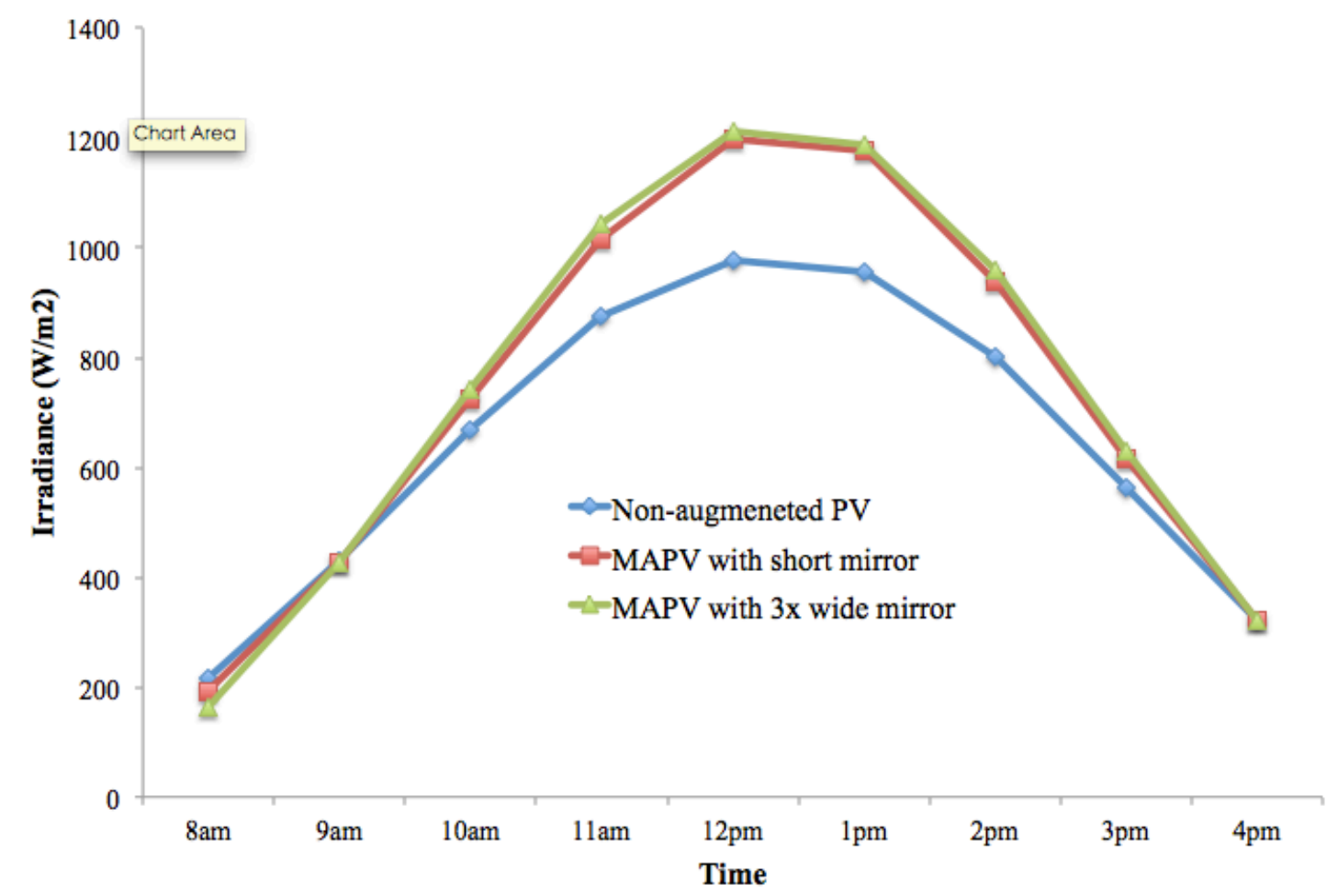

Figure 28: Average irradiance comparisons between MAPV and non-MAPV from raytrace result.

Figure 29 shows one I-V curve snapshot of a very specific time from the Daystar multi-tracer at the field-test site. The I-V curves compare PV modules with and without mirror augmentation as measured at the outdoor test facility. The modules are mounted at $55^{\circ}$ and the mirror mounted at $0^{\circ}$. This configuration is expected to optimize augmentation during the winter when the sun's elevation angles are lower. The power output on the fixed MAPV panel is $223.8 \mathrm{~W}$ compared to $199.4 \mathrm{~W}$ of the non-augmented panel. 


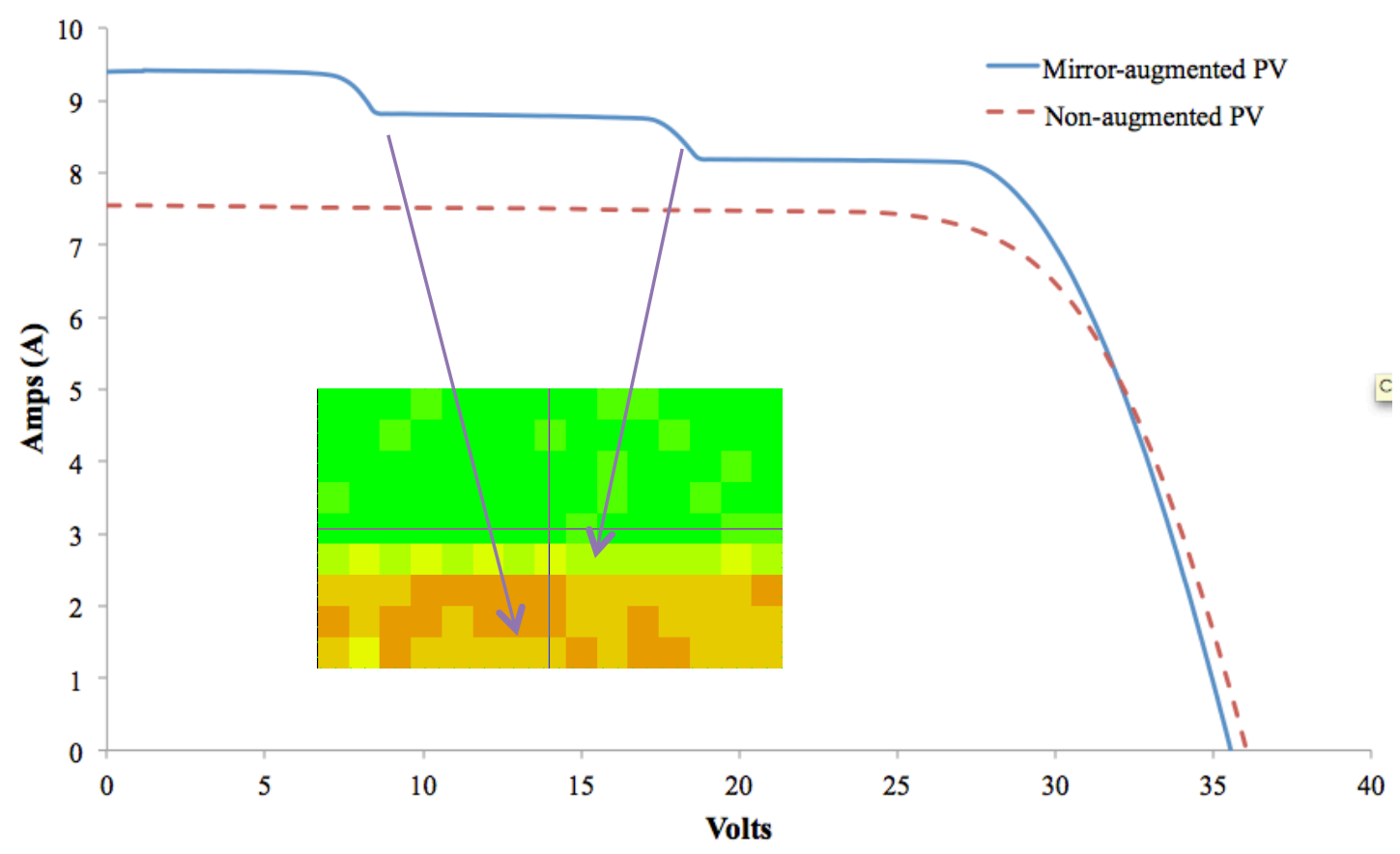

Figure 29: I-V curve result from the field-test site at Replex Plastics. The inset irradiance map shows where the by-pass diodes operate.

\subsection{Power Production from Fixed MAPV Over a 3 Month Period}

In addition to the I-V snapshot, power production data was recorded for a three month period from January $6^{\text {th }}$ to March $31^{\text {st }}$, 2012. Over this limited period of study, the MAPV system produced $8 \%$ more power than an equivalent non-augmented module tilt at $55^{\circ}$ and boosted $10 \%$ more power than the module tilt at $34^{\circ}$ as shown in Table 4. Figure 30 demonstrates the fixed MAPV construction installed at Replex Plastic's test site near Columbus, OH. From the photograph, the Gen 0 model indicates nonaugmented PV and the Gen 2 model indicates MAPV. This photograph also shows that the module and mirror of MAPV were tilted at $55^{\circ}$ and $0^{\circ}$, respectively. 
Table 4: Power production of each month at field-test site*

\begin{tabular}{|c|c|c|}
\hline Month $\backslash$ Modules & MAPV 55\% $/ \mathrm{PV} 55^{\circ}$ & \\
\hline January** & $109 \%$ & $122 \%$ \\
\hline February & $110 \%$ & $114 \%$ \\
\hline March*** & $106 \%$ & $103 \%$ \\
\hline Total & $108 \%$ & $110 \%$ \\
\hline
\end{tabular}

*The field-test data was acquired by Dave Hollingshead.

**Data acquisition began on January $6^{\text {th }}, 2012$

***Data shown is through March 31 ${ }^{\text {st }}, 2012$

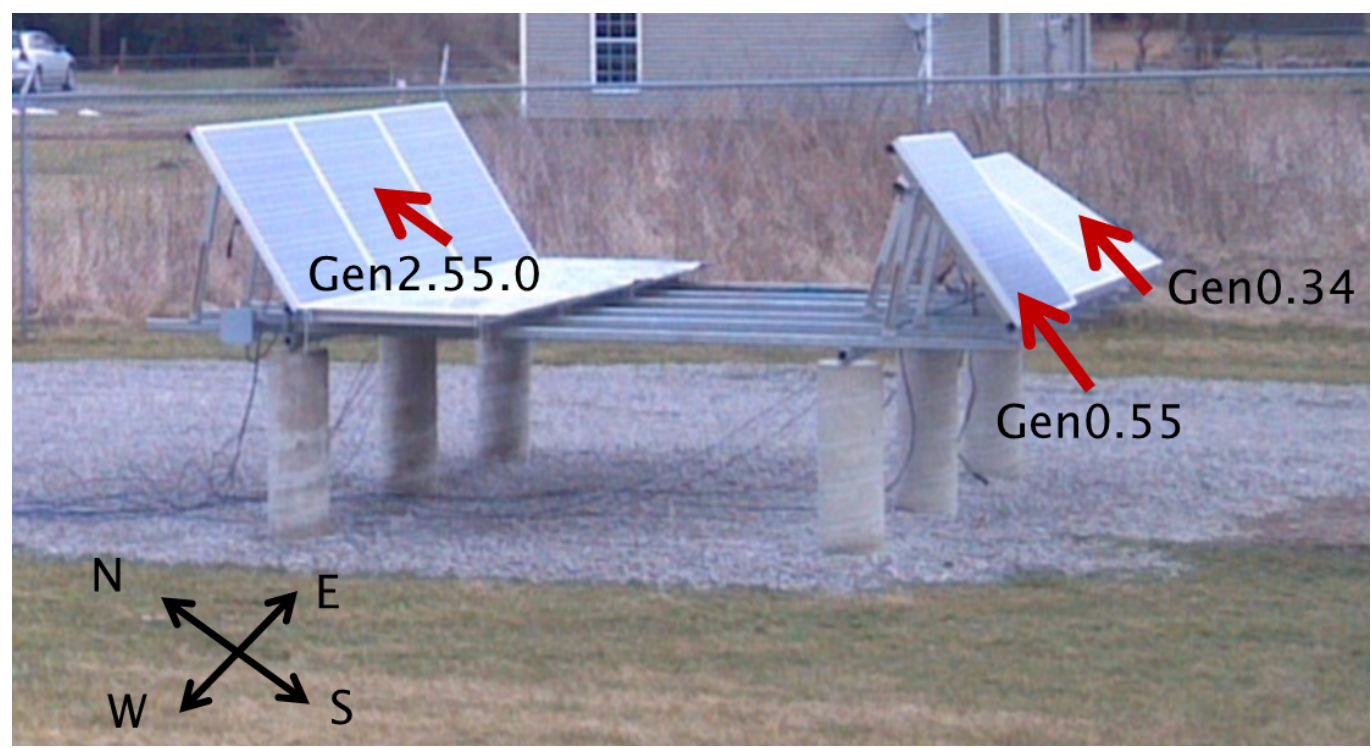

Figure 30: The fixed MAPV test system at Replex Plastics (latitude: 40.38, longitude: 82.48). 


\subsection{Performance on Annual Power Production}

After obtaining the real-world data, the yearly power output of fixed MAPV needed to be explored. The performance of fixed MAPV was investigated for both outdoor fieldtest and optical analysis. For the outdoor field-test site, an adjustable mounting system "time machine" was used to estimate yearly power production. Figure 31 shows the prototype of the "time machine" apparatus to which the PV panel and solar mirror were mounted on an adjustable metal frame. For the 3D optical analysis, a $3 \mathrm{X}$ wide mirror model was used to simulate the outdoor field-test. The $15^{\text {th }}$ day of each month was chosen to represent the power output for each month. In addition, a noon sun was chosen as representative irradiance for each day. Figure 32 demonstrate the relative position between 3D geometric model and selected suns of each month at noon.

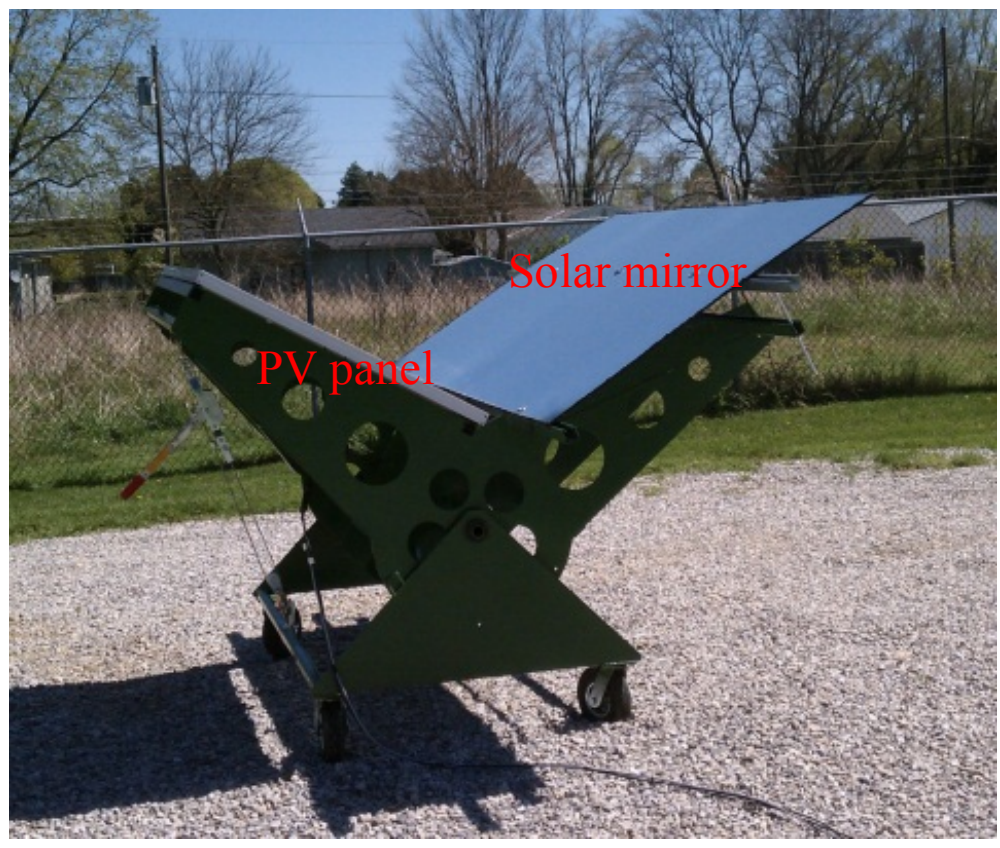

Figure 31: The prototype of time machine apparatus. 


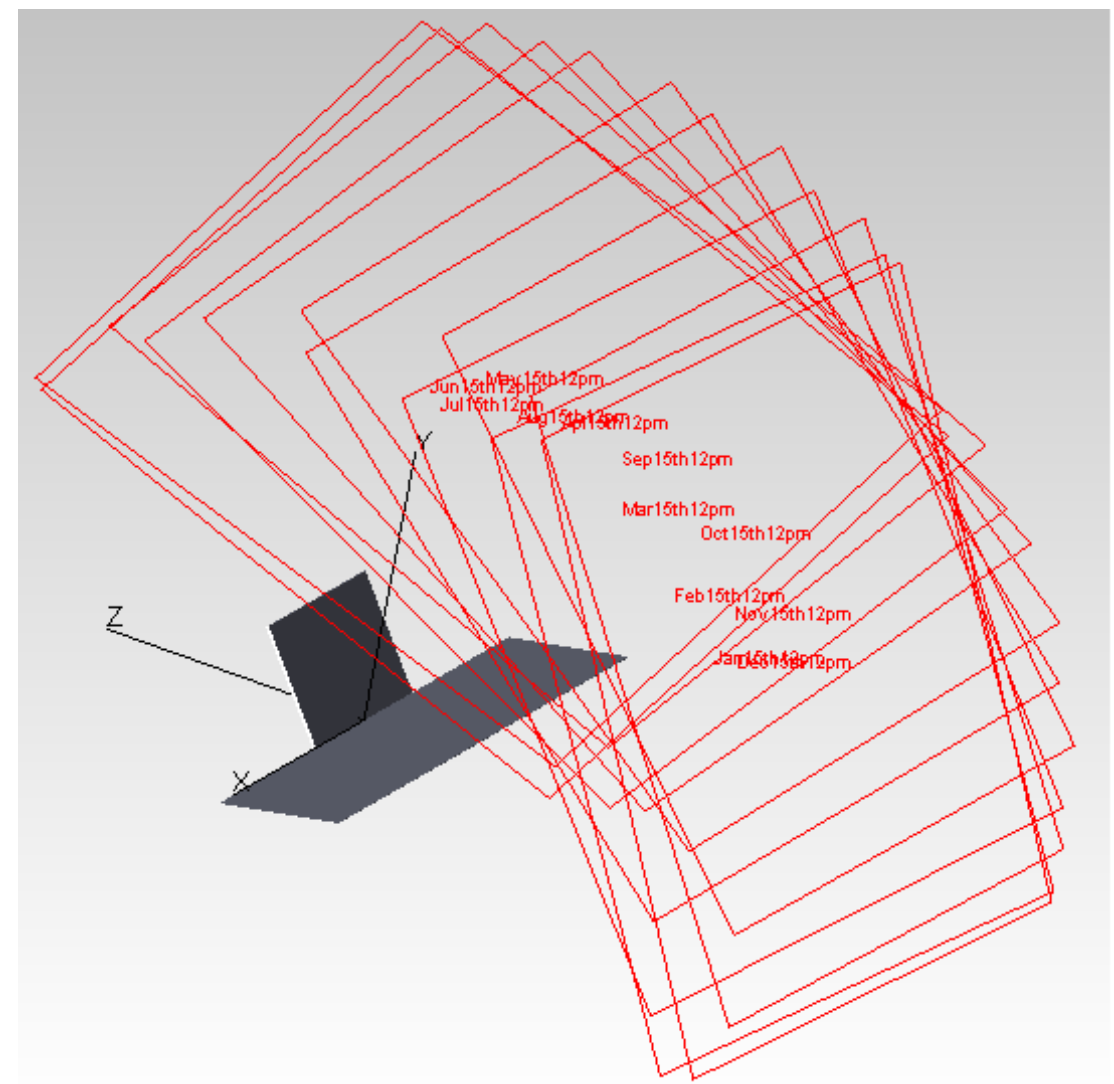

Figure 32: Relative position between the $3 \mathrm{X}$ wide mirror model and the sun from the $15^{\text {th }}$ of each month

Figure 33 shows the ray-trace result of Gen2.50.10 model along with real world data from "time machine". Dave Hollingshead acquired the power output data from "time machine"49. The x-axis represents the selected day of each month. The left y-axis shows the irradiance received from PV panel in 3D optical analysis. The right $y$-axis shows the energy output generated from the "time machine". 


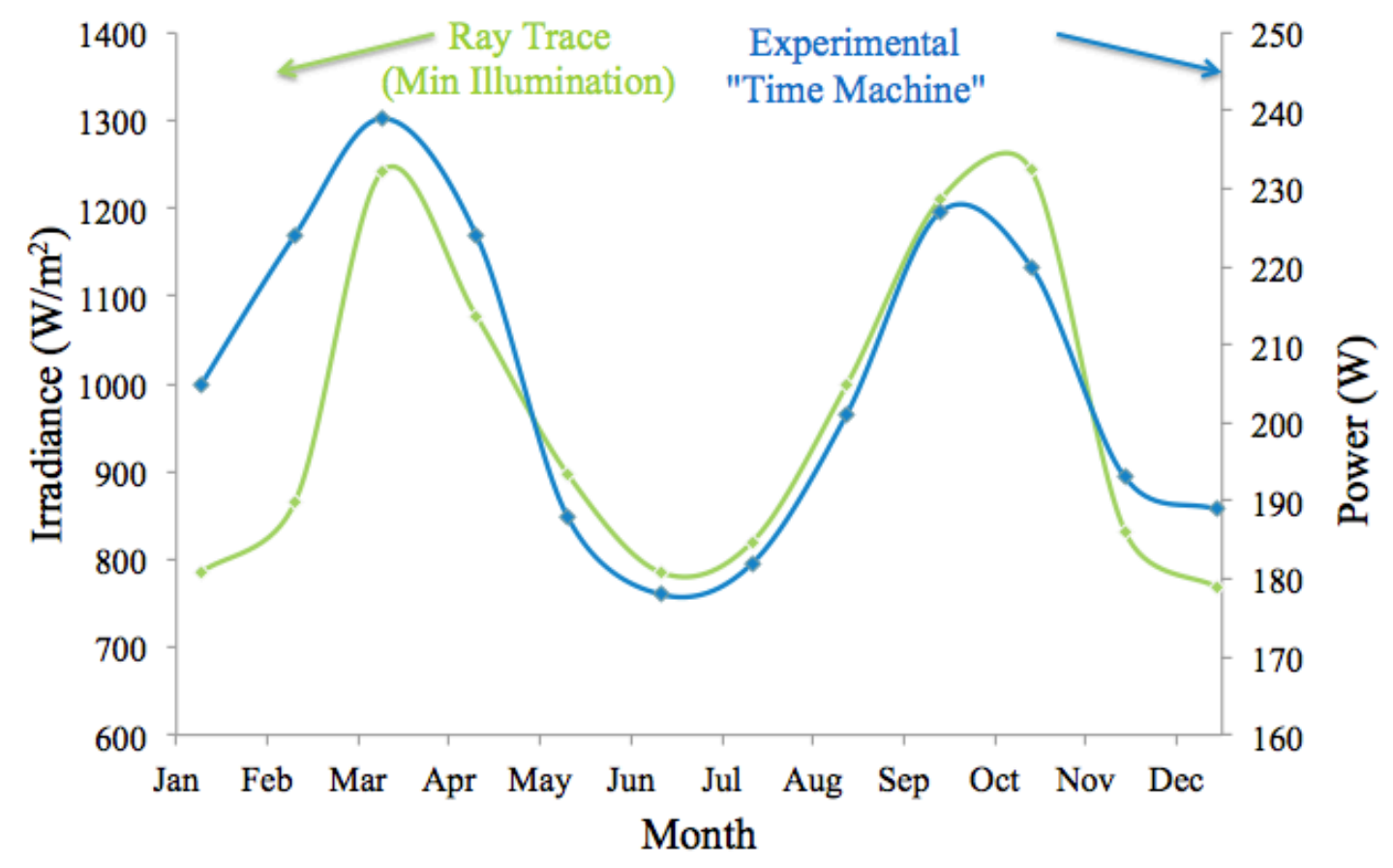

Figure 33: Estimated power output of Gen2.50.10 model by month.

\subsection{The Effect of Mirror Tilt Angle on Uniformity and Irradiance Value}

In order to investigate how the mirror tilt angles affect the irradiance uniformity, a series of simulations were performed to generate the irradiance distribution and also to acquire the average irradiance value by using optical analysis. In these simulations, the module tilt angle was fixed at $55^{\circ}$ and the $15^{\text {th }}$ day of each month was selected as representative samples to observe irradiance distribution. Mirror tilt angle was the only parameter varied in the simulation, from -5 to 35 degree. Table 5 shows the average irradiance on the PV panels (values with background colors) and how the models perform on uniformity. 
Table 5: A series simulation of mirror tilt angle and its performance on the irradiance map and the average irradiance value $\left(\mathrm{W} / \mathrm{m}^{2}\right)$.

\begin{tabular}{|c|c|c|c|c|c|c|c|c|c|c|}
\hline Mon. & Ele. angle & Gen2.55.-5 & Gen2.55.0 & Gen2.55.5 & Gen2.55.10 & Gen2.55.15 & Gen2.55.20 & Gen2.55.25 & Gen2.55.30 & Gen2.55.35 \\
\hline Jan 15th & 27.79 & 1254.5 & 1394.4 & \begin{tabular}{|l}
1336.2 \\
\end{tabular} & 1261.6 & 1183.4 & 1106.3 & 1033.4 & 956.32 & 866.57 \\
\hline Feb 15th & 35.92 & 1121.4 & 1259.8 & 1413.3 & 1373.8 & 1301.1 & 1226.5 & 1148.5 & 1074.6 & 1005.4 \\
\hline Mar 15th & 46.91 & 965.29 & 1075.2 & 1222.8 & 1352.5 & 1429 & 1360.9 & 1289 & 1214.9 & 1137.1 \\
\hline Apr 15th & 58.98 & 901.43 & 901.43 & 984.36 & 1118.8 & 1265.5 & 1386 & 1387.9 & 1326 & 1255.4 \\
\hline May 15th & 67.95 & 817.26 & 817.26 & 817.26 & 913.41 & 1046.6 & 1169.6 & 1310.7 & 1324 & 1288.9 \\
\hline Jun 15th & 71.78 & 750.09 & 750.09 & 750.09 & 792.17 & 992.47 & 1045 & 1182.9 & 1281.1 & 1221.8 \\
\hline Jul 15th & 69.51 & 762.19 & 762.19 & 762.19 & 833.55 & 961.02 & 1098.6 & 1213.9 & 1260.1 & 1229.7 \\
\hline Aug 15th & 62.34 & 858.74 & 858.74 & 894.44 & 1028.2 & 1166.7 & 1297.3 & 1354.1 & 1318.8 & 1252.6 \\
\hline Set 15th & 51.87 & 960.11 & 1003.4 & 1140.8 & 1290.9 & 1416.4 & 1430.7 & 1361.4 & 1288.5 & 1213.4 \\
\hline Oct 15th & 40.63 & 1071 & 1221.6 & 1357.9 & 1469.9 & 1398.6 & 1323.6 & 1246.9 & 1167.6 & 1091.7 \\
\hline Nov 15th & 30.81 & 1221.3 & 1378.3 & 1405.3 & 1330.4 & 1253.9 & 1174.2 & 1098.3 & 1027.1 & 938.47 \\
\hline Dec 15th & 26.03 & 1288.5 & 1392.7 & 1318.7 & 1242.7 & 1163.1 & 1087.1 & 1016.5 & 928.82 & 838.69 \\
\hline \multicolumn{2}{|c|}{ Degree of uniform } & good & normal & bad & \multicolumn{2}{|c|}{ Max. Power Output } & & & & \\
\hline
\end{tabular}

The red number shows the maximum average irradiance values for each month (row). It indicates that one certain model configuration is the best for obtaining maximum power. In Table 5, different colors were used to represent the degree of uniformity. The method used to define uniformity was to observe the ratio of the non-uniform area to the whole panel area using an irradiance map. Figure 34 shows the ray-trace result of Gen 2.55.25 when the mirror tilt angle is at $25^{\circ}$ on July $15^{\text {th }}, 2012$. In Figure 34a, the irradiance map shows uniform intensity between 1100 and $1300 \mathrm{~W} / \mathrm{m}^{2}$. The average irradiance value for this panel was $1213.9 \mathrm{~W} / \mathrm{m}^{2}$. From the light's pathway in Figure 34b, the reflected light (blue rays) can fully illuminate the panel and result in uniform irradiance distribution (green in Table 5). 


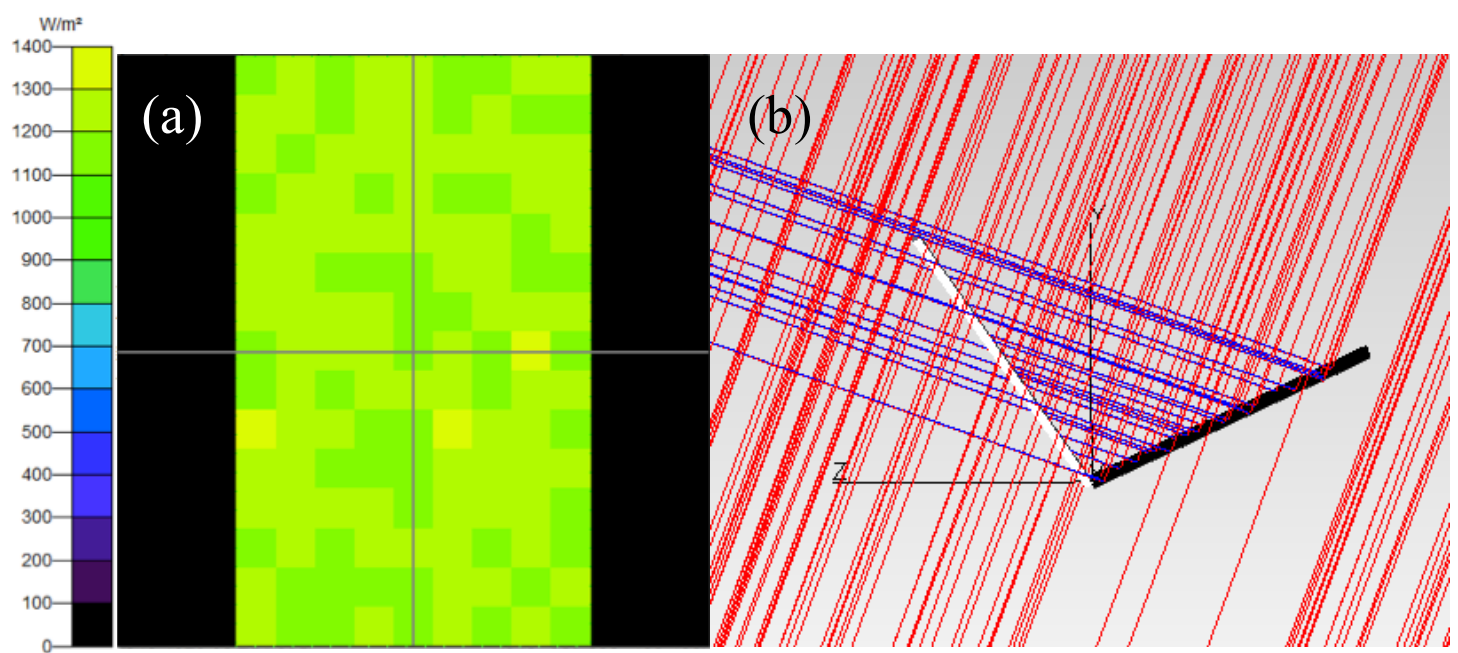

Figure 34: Ray-trace result of Gen2.55.25 on July $15^{\text {th }}$ shows uniform irradiance distribution (a) and its light pathway (b).

When the mirror was further tilted to $30^{\circ}$, a non-uniform irradiance pattern formed caused by mirror-augmentation (Figure 35a). A non-uniform area occurred on the left side of the panel where the region was not augmented by the mirror. The average irradiance value of this panel was $1260 \mathrm{~W} / \mathrm{m}^{2}$. The irradiance value of non-augmented region was below $1100 \mathrm{~W} / \mathrm{m}^{2}$ whereas the mirror-augmented region was above 1300 $\mathrm{W} / \mathrm{m}^{2}$. The ray-trace result (Figure 35b) shows that the reflected light couldn't disperse uniformly on the panel. The slight non-uniformity of Gen2.55.30 model was defined as blue in Table 5 to indicate that the non-uniformity grade is less than one third of the panel area. 


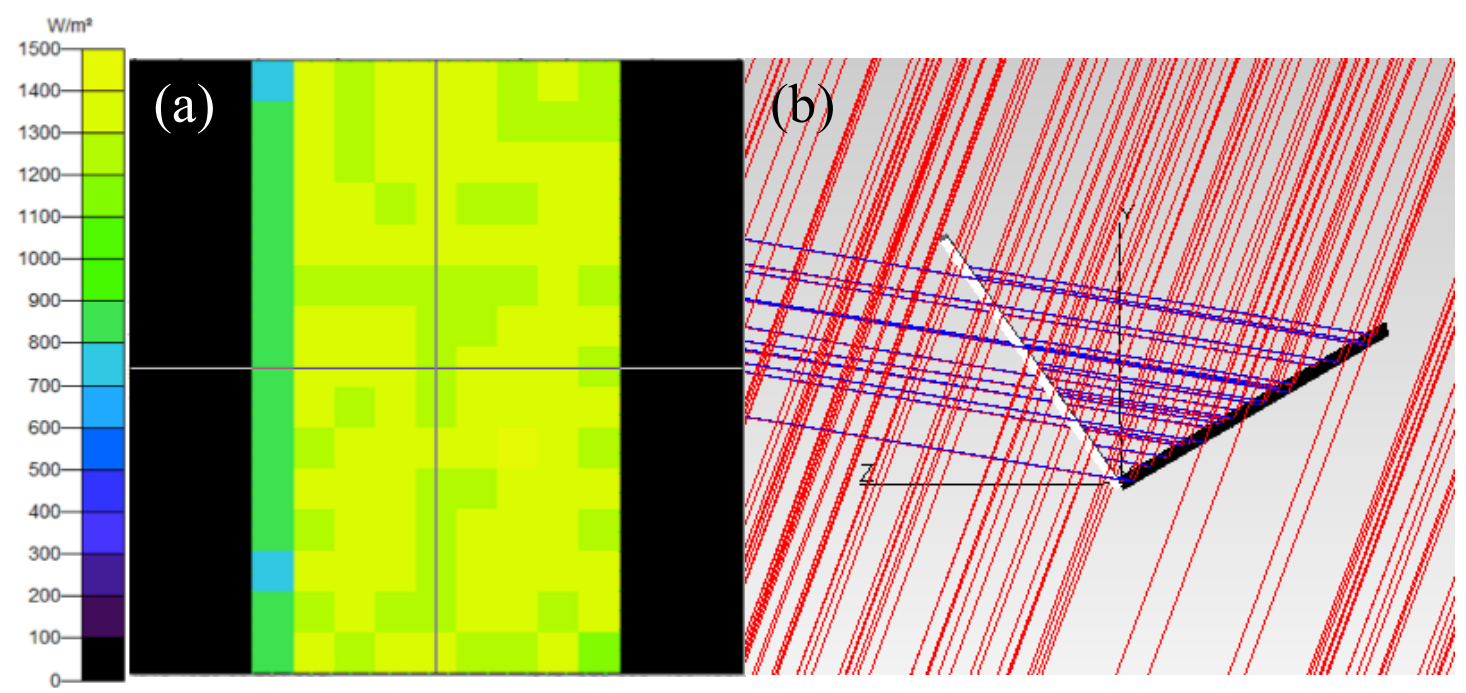

Figure 34: The ray-tracing results of Gen2.55.30 on July $15^{\text {th }}$, which shows a nonuniform region occurring on the PV panel (a) and the pathway of light (b).

A significant non-uniformity occurs when the non-uniform region is greater than one third of the total panel area. An example was shown is Figure 36 when the mirror tilt angle reached $35^{\circ}$. It is obvious that there are three kinds of colors in irradiance map (Figure 36a). The yellow region indicates the panel area, which was enhanced by the reflected rays. The blue region indicates a non-augmented panel area where irradiance value is below $800 \mathrm{~W} / \mathrm{m}^{2}$. The green region shows the pixel area was partial augmented by mirror. The average irradiance value of whole panel is $1229.7 \mathrm{~W} / \mathrm{m}^{2}$. The light's pathway confirmed the non-uniformity shown in irradiance map (Figure 36b). The orange was used to represent the severe condition of non-uniformity in Table 5. 


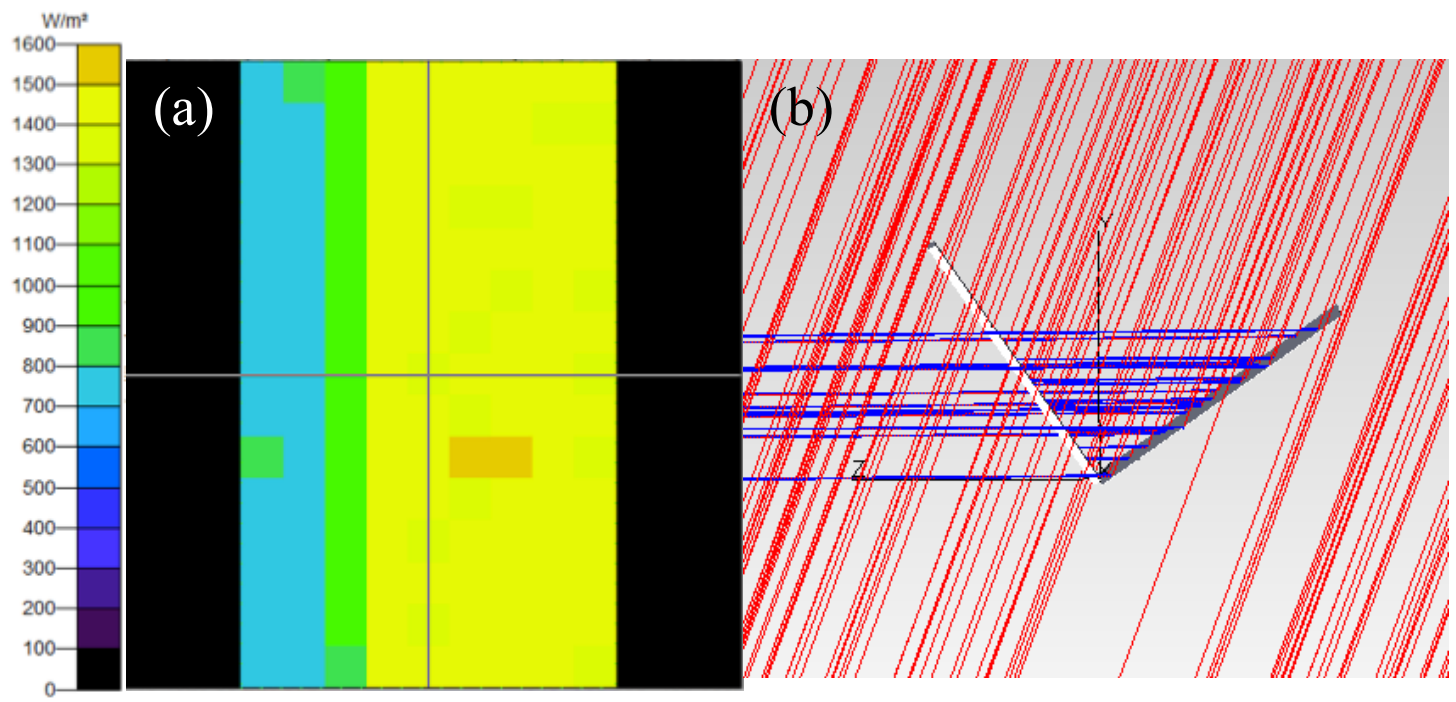

Figure 36: Ray-trace result of Gen2.55.35 on July $15^{\text {th }}$ shows a significant non-uniform region on the panel (a) and the pathway of light (b).

In Table 5, the irradiance values highlighted in red represent the maximum output in each month. From the perspective of power output, it is obvious that mirror tilt angles stay low in winter and increase to 30 degree in summer for maximum value. The results in Table 5 show that the "gullwing curve" can be solved by tuning the mirror angles during different parts of the year. Figure $\mathbf{3 7}$ demonstrates the average irradiance values for each model configuration throughout a year. It can be seen that both the Gen2.55.-5 and the Gen2.55.0 (first and second models in Table 5) show a concave curve. These two models reach a maximum value in January and December and reach a minimum value in June. Gen2.55.5 starts to form a "gullwing curve" and has partial low value in January and December. From Gen2.55.10 to Gen2.55.25, the "gullwing curve" becomes obvious because the relative minimum power output occurs in January and December. For the Gen2.55.10, the minimum irradiance value occurs on June, but the minimum irradiance value for the Gen2.55.25 is during the month of on December. 


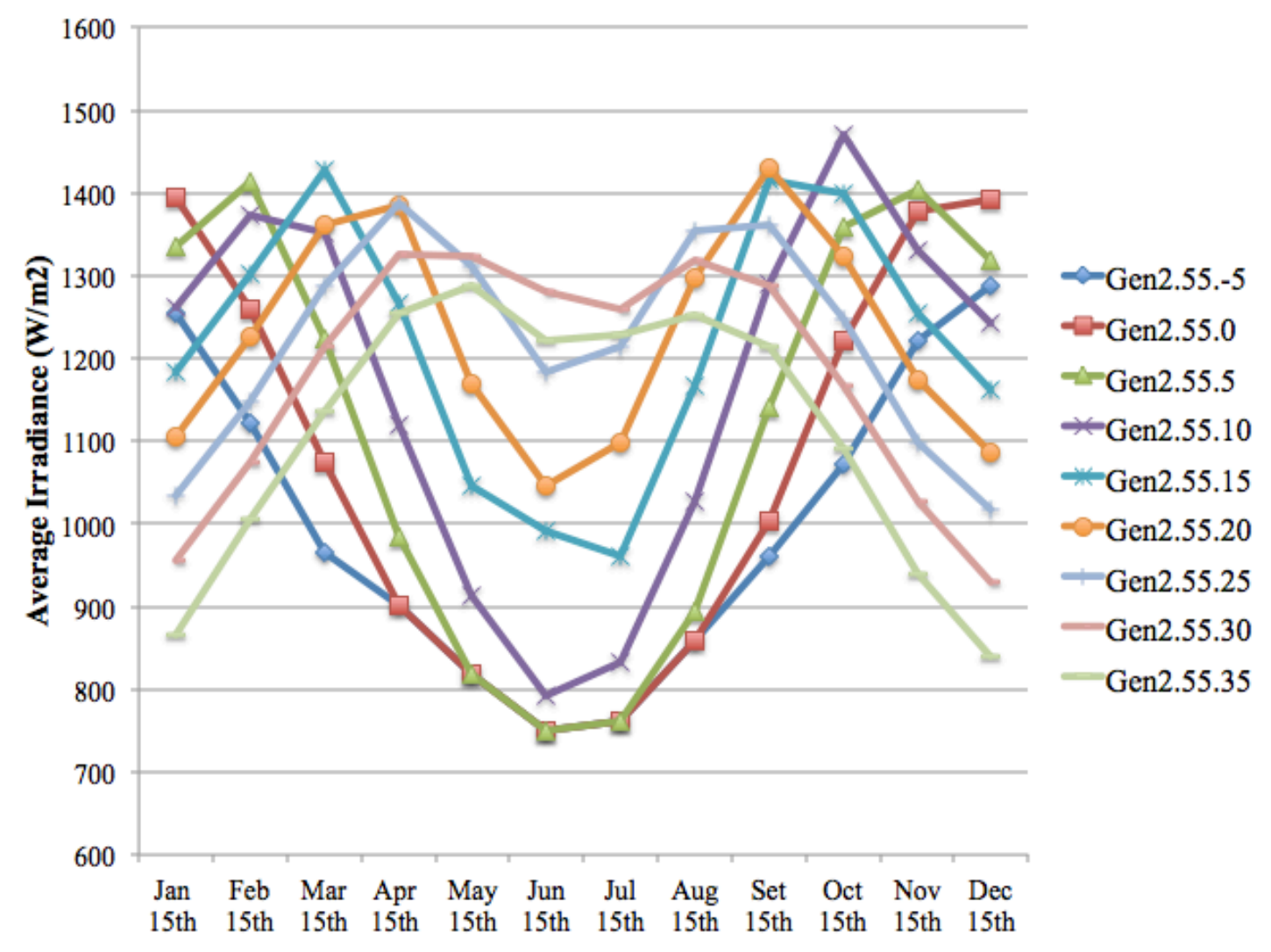

Figure37: The irradiance values throughout a year from various model configurations.

\subsection{Possible Solutions for the "Gullwing Curve"}

From the results shown in the previous sections, the "gullwing curve" is disadvantageous for a fixed MAPV system. One possible solution to improve the phenomenon of the "gullwing curve" on yearly power production is to change the mirror shape in order to increase power production in the summer months and maintain good irradiance distribution on the PV panel. A new design was proposed to bend the mirror from a flat to a barrel shape as shown in Figure 38. Two different radii of curvature were used to compare the ray-trace results with flat mirror module results. Figure 39 shows the ray-trace results for a barrel mirror (Gen3) model with 90 inches radius of curvature on January $10^{\text {th }}, 2012$. The average irradiance value on this panel was $1190 \mathrm{~W} / \mathrm{m}^{2}$. The 
light's pathway in Figure 38b indicates part of incident rays were reflected to the sky instead of concentrating on the panel. The non-uniformity issue exists because the irradiance map has non-augmented and mirror-augmented regions.

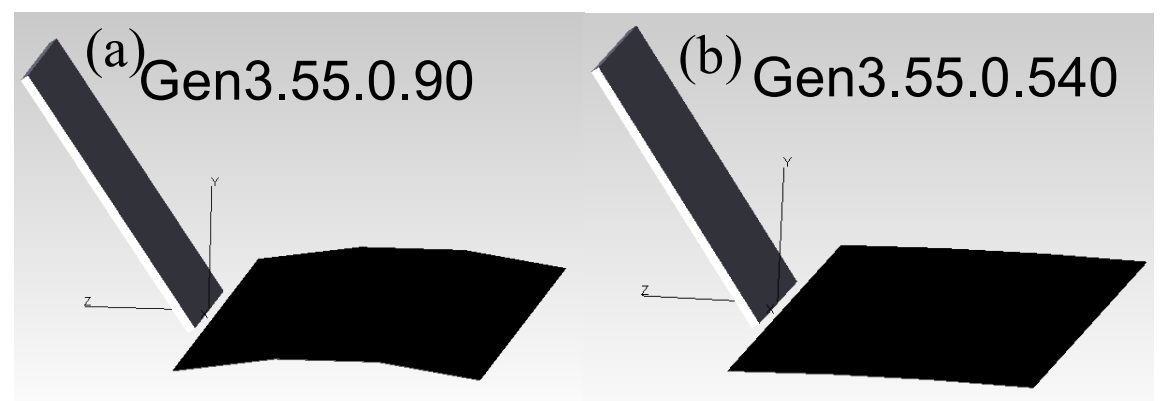

Figure 38: A new MAPV design of a barrel mirror with (a) 90 in. radius of curvature, and (b) with a $540 \mathrm{in.}$ radius of curvature.

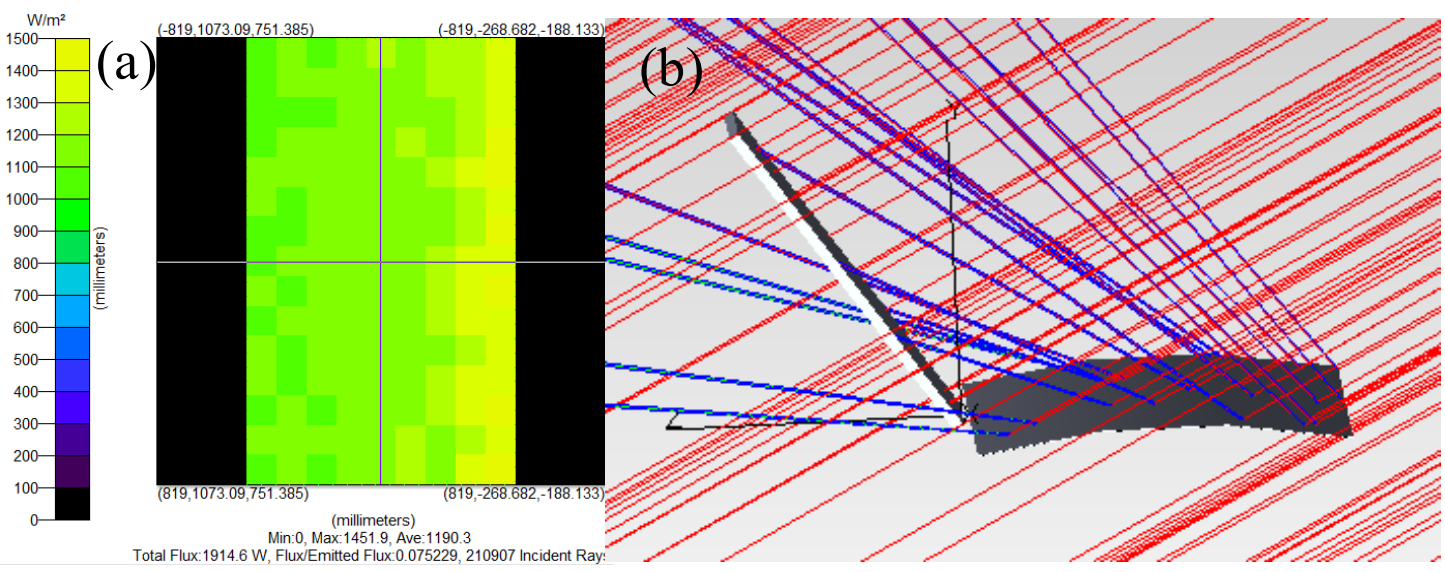

Figure 39: The ray-trace result of the Gen3.55.0.90 model at noon on January $10^{\text {th }}, 2012$,

(a) irradiance map and (b) the pathway of light.

Figure 40 shows the ray-trace result for a barrel mirror (Gen3) model with a 540 inches radius of curvature. The average irradiance value of this model is $1325.7 \mathrm{~W} / \mathrm{m}^{2}$ (Figure 40a). The ray-trace results shows that this model can reflect more incident light on the panel compared to the Gen 3.55.0.90 model (Figure 40b). The irradiance map reveals non-uniformity where half of the panel is augmented by the reflected rays. 


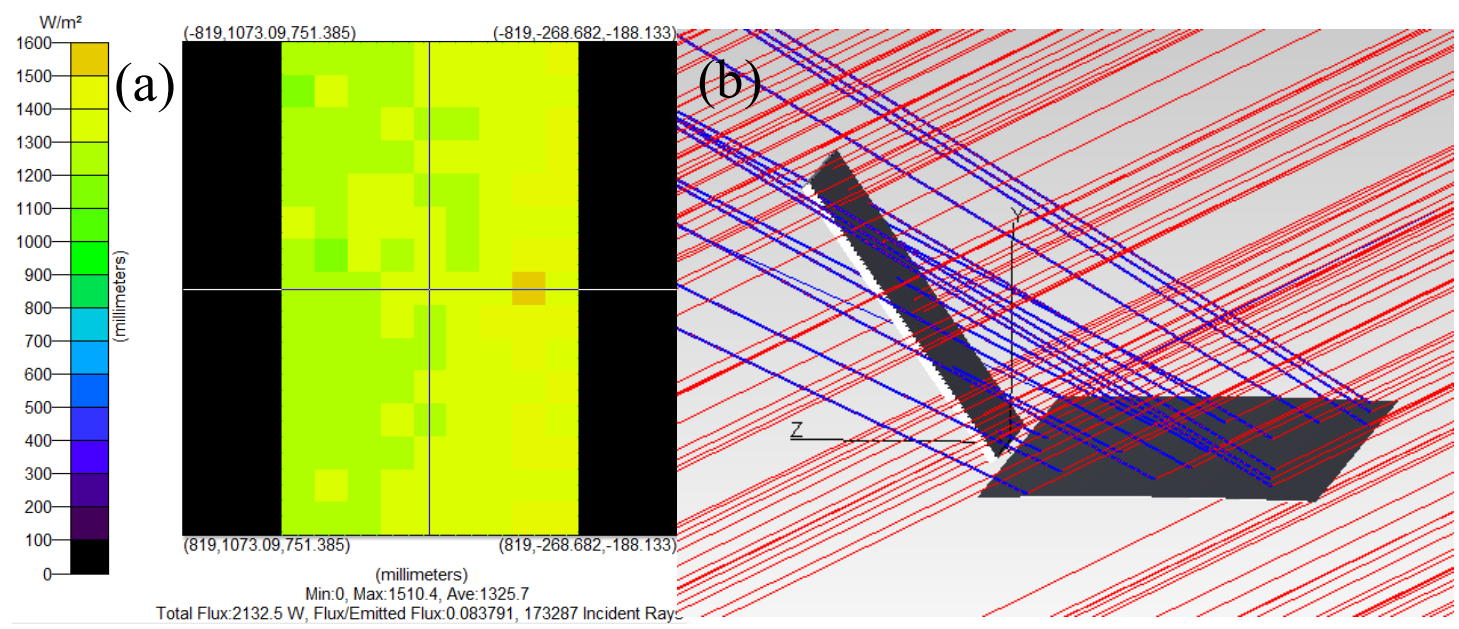

Figure 40: The Ray-trace result of the Gen3.55.0.540 model at noon on January $10^{\text {th }}$, 2012 (a) irradiance map and (b) the pathway of light.

Another possible mirror design is to use a concave mirror in the MAPV system.

Figure 41 illustrates the concave mirror (Gen4) model with a 100 inches radius of curvature (b) and its ray-tracing result on March $10^{\text {th }}, 2012$ at noon (a). The concave mirror concentrates light on a small region of the PV panel and leads to severe nonuniformity. The average irradiance value for this model was $1408.7 \mathrm{~W} / \mathrm{m}^{2}$.
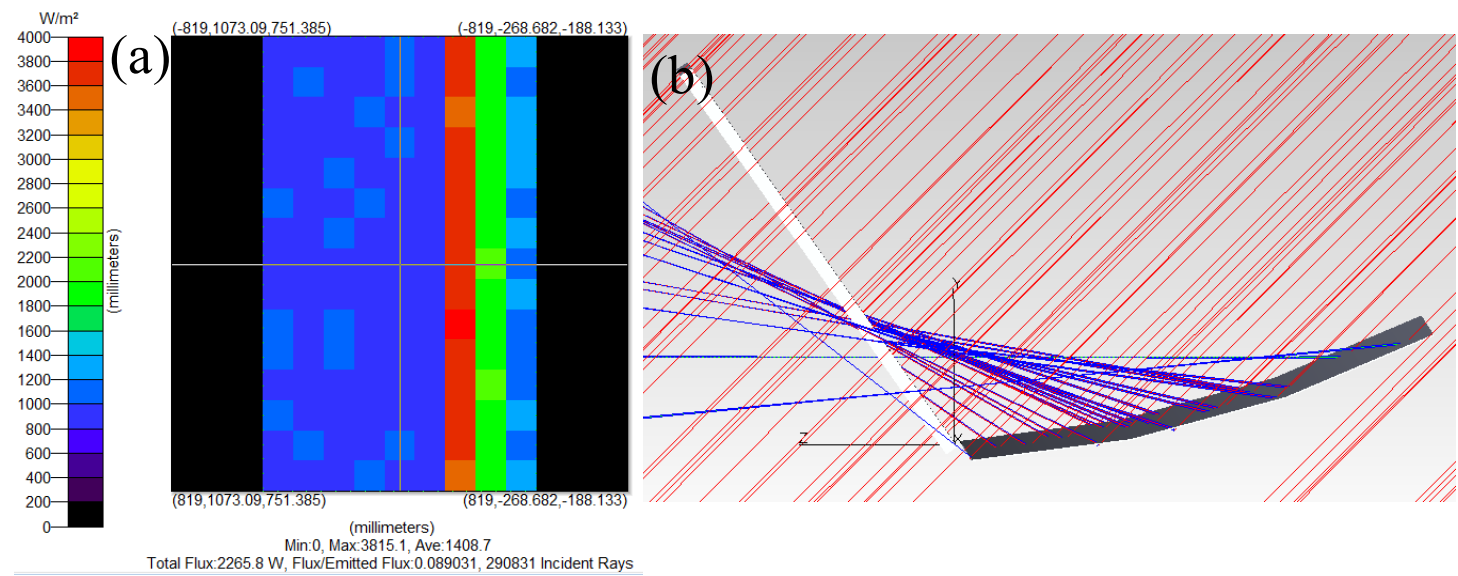

Figure 41: The ray-trace result of the Gen4.55.10.100 model at noon on March $10^{\text {th }}, 2012$ (a) irradiance map and (b) the pathway of light. 
Figure 42b illustrates another sample of a concave mirror (Gen4) model with a 900 in. radius of curvature on March $10^{\text {th }}, 2012$ at noon. The ray-trace result (Figure 42a) shows that the non-uniformity issue could not be resolved even with the increase of the radius of curvature to 900 inches (nearly flat). The average irradiance value of this model was $1413 \mathrm{~W} / \mathrm{m}^{2}$.
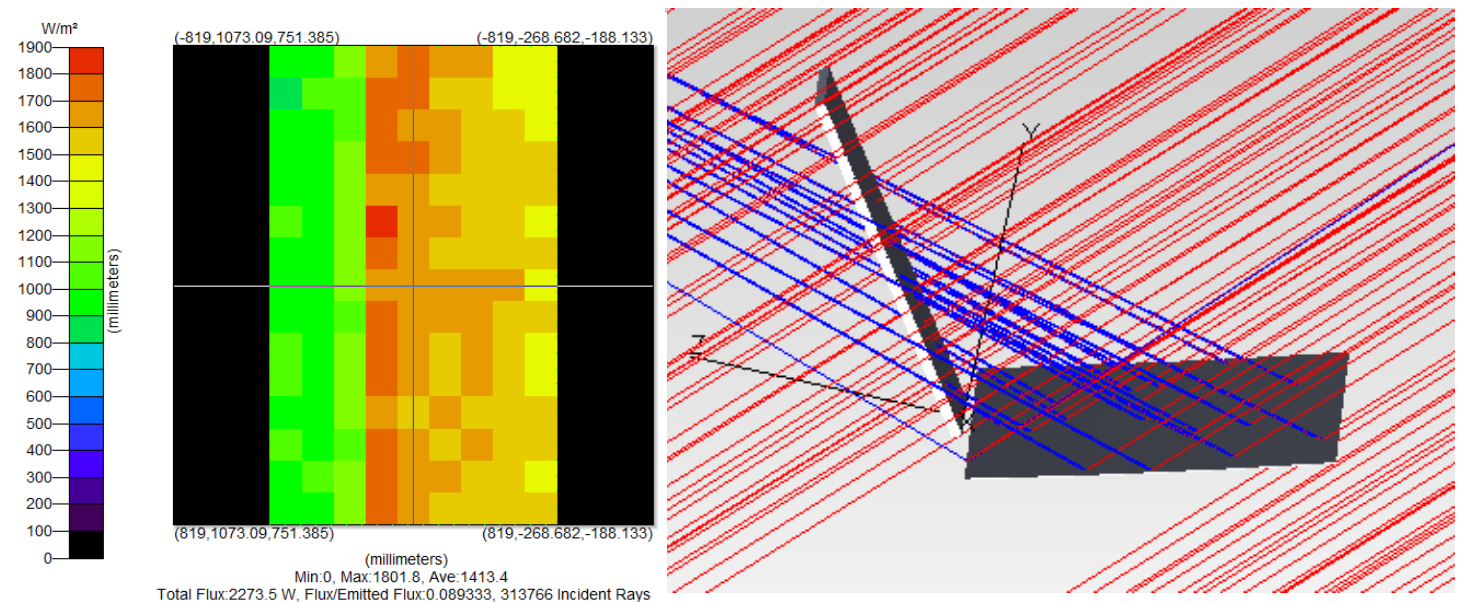

Figure 42: The ray-trace result of the Gen4.55.10.900 model at noon on March $10^{\text {th }}, 2012$ (a) irradiance map and (b) the pathway of light.

\section{Discussion}

This section discusses the results between model simulations and the outdoor fieldtest data.

\subsection{Maximum Yearly Power Production for Fixed MAPV}

The best model configuration for fixed MAPV for yearly power output is for the tilt angle of the PV module to be $50^{\circ}$ and solar mirror tilt angle to be $10^{\circ}$. This Gen 2.50 .10 model for fixed MAPV provides the maximum yearly output when the energy output is integrated over an entire year. The sun's elevation angles vary over time (Table 5); 
therefore, the model configuration should be fine-tuned daily to obtain maximum power output for each day in theory. In other words, the power generated by fixed MAPV system is not necessarily the maximum output for each day, but is the maximum power over a year and possibly over the lifetime of the system. It indicates that the "gullwing curve" may happen for each model configuration when the yearly power production is investigated. The best model configuration will be used for the indoor 3D optical simulations. As for outdoor field-test site, different model configurations may be used in order to obtain optimal power output during a particular time of the year.

\subsection{Mitigation of Non-Uniform Irradiance in Fixed MAPV}

The model configuration used to perform the 3D optical analysis was based on the 1D calculation results discussed in section 4.1. The ray-trace result for the Gen2.50.10 model showed three different kinds of non-uniformity. The first type of uniformity issues was a "shadow effect" caused by the mirror shadowing in the early morning. The "shadow effect" occurred because of the low elevation of the sun and the mirror was tilted at certain angle (Figure $\mathbf{1 8}$ and 24). The "shadow effect" is inevitable when the sun's position is lower than mirror tilt angle, but its effect negligible because of the low intensity of the sun at that time. It is expected that a similar phenomenon will occur during sunset when the sun's position will again be lower than the mirror tilt angle.

The second kind of non-uniformity was the "end effect". The irradiance maps at 9 a.m. and 4 p.m. showed that the panels were fully illuminated and the mirror did not add additional illumination to the PV panel. From 10 a.m. through 3 p.m., the irradiance pattern indicates that the mirror did not augment a small region (Figure 19-22). Due to the pathway of the sun, the relative position between sun and MAPV system is not 
always optimal. The "end effect" formed at 12 p.m. and the irradiance maps are symmetric around noon from 10 a.m. to 3 p.m. because of the similar elevation angles of the sun (Figure 23).

Both of the "shadow effect" and "end effect" occurred on a small area of the PV panels. From the perspective of the whole panel, another non-uniformity caused by the mirror-augmentation can be observed. From 10 a.m. to 3 p.m., the non-uniformity in the irradiance pattern caused by mirror augmentation occurred at the bottom region of each model. The augmented region reached maximum area at 12 p.m., which indicates that the module obtained maximum power output at noon. The goal of the fixed MAPV system was to obtain maximum energy output and uniform irradiance distribution, which did not occur with these model designs; therefore, the $2^{\text {nd }}$ and $3^{\text {rd }}$ type of non-uniformity issues need to be studied further.

In order to solve the non-uniformity issue caused by the "end effect", a $3 \mathrm{X}$ wide mirror was designed to compensate for the corner that was not augmented as shown in Figure 26. After ray-tracing the $3 X$ wide mirror model, Figure 27 demonstrates the irradiance maps from 8 a.m. to 4 p.m. It can be observed that the "shadow effect" on 8 a.m. model becomes severe because of the long mirror. The "shadow effect" can be ignored because of the low irradiance values in the early morning and in the evening. With the implementation of longer mirrors, the "end effect" was successfully removed (Figure 27). The irradiance distribution on mirror-augmented region was uniform from 10 a.m. to 3 p.m.; therefore, tuning the mirror size and tilt angle can change the irradiance distribution. 
The irradiance maps are divided into two regions, one the mirror-augmented region and another is non-mirror augmented region from the irradiance maps at 10 a.m. to 3 p.m. This $3^{\text {rd }}$ type of non-uniformity issue may result in fast degradation on certain portion of the panel. For the non-uniformity caused by mirror-augmentation, a new mirror design was needed to solve this problem since none of the previous model designs could mitigate the non-uniformity caused by mirror-augmentation.

\subsection{Power Production Improvement of Fixed MAPV for Field-Tests}

From the ray-trace results in Figure 28, it can be concluded that the 3X wide mirror panel system did not vastly increase the irradiance compared to the short mirror design. Therefore, a mirror with a length shorter than the $3 \mathrm{X}$ wide mirror panel system could be used to reduce the cost of the mirror. In addition, the MAPV system had a boost of $20 \%$ irradiance over the non-augmented PV at noon. It can be estimated that the overall enhanced irradiance of MAPV module is between $10-20 \%$ for an entire day. The I-V curve snapshot in Figure 29 also confirms the total energy output enhanced by the mirror was similar to ray-trace result. It was obvious that the three steps in the MAPV I-V curve (Figure 29) indicate that the module by-pass diodes were operating, which indicates that the illumination is non-uniform across the panel. This non-uniformity caused by mirror augmentation is classified as the $3^{\text {rd }}$ type of non-uniformity, which could cause fast degradation of the module. Further investigation is needed to understand whether this system would actually induce rapid degradation of the module or whether the benefit of a $20 \%$ increase in irradiance is more beneficial than the possible decrease in lifetime of the system. 


\subsection{Differences between Snapshot Power Production and Field-Test Power}

\section{Production}

After a snapshot comparison between the ray-trace result of the best MAPV model configuration (Gen2.50.10) and the I-V curve, a continuous power output from field-test site over the three month period was used to record the power enhancement by MAPV. From the results shown in Table 4, Gen2.55.0 model produced $8 \%$ more power than Gen 0.55 model and boosted $10 \%$ more power than Gen 0.34 model. Several factors may play a role in why there are a power differences between the power outputs from the field-test site and the indoor simulation and I-V curve snapshot. The field-test power production may be lower than the model's predicted power production due to several variables that were not included in the model. One reason that the PV panels can receive lower irradiance from the sun is because of unpredictable weather conditions. The modeled power production was based on a perfectly sunny day and did not account for the various weather conditions seen in the real-world. Another possible reason for the discrepancy in power production is because the Gen2.55.0 was not necessarily the best model configuration for the months of January through March. The elevation angle of the sun is different from January to March, so the mirror tilt angle should be tuned in order to obtain maximum power output. In addition, the back surface acrylic mirrors used at fieldtest site may degrade under outdoor exposure. The reflectance of the solar mirror is not necessarily $88 \%$ as it is assumed in optical analysis ${ }^{50}$. The PV panels were set as perfect absorbers in the model; however, the PV panels are not actually perfect absorbers. The wavelength used in model simulations is at $546 \mathrm{~nm}$; however, the PV panels at field-test 
receive a broad range of wavelength. All of these factors contribute to lower power production at field-test site compared to the modeled power production.

The fixed MAPV system used at the field-test site consisted of three consecutive PV panels coupled with three solar mirrors of the same length (Figure 30). An end effect is expected at the corner of panel because the three consecutive mirrors did not extend past the edge of PV panels on both sides. This end effect can easily be removed with design changes such as increasing the length of the mirrors (Gen2 with longer mirrors). The end effect is rather small so an analysis of the additional cost for material for the addition of longer mirrors needs to be completed compared to the possible degradation effects due to the non-uniform illumination by this small end effect.

\subsection{A Gullwing Curve on Annual Power Production}

The blue curve in Figure 33 represents the power production measured by the "time machine" and the green curve represents the ray-trace result from the 3D optical analysis. In Figure 33, the fixed MAPV had a minimum power output in the summer and obtained a maximum output during the equinox. Both the estimated power output and the experimental results confirmed that a "gullwing curve" in power production was present. The "gullwing curve" occurred due to a minimum energy output in the summer (Figure 43) because the reflected light did not fully illuminate the desired PV panel due to the high elevation angle of the sun during the summer. This indicated that the low power output in the summer was due to the mismatch between the module and mirror tilt angles and the position of the sun. Even though, the fixed MAPV system still produced higher power than the power generated by the non-MAPV system during the summer months. The Gen2.50.10 model (Figure 33) was capable of increasing the power production in 
the winter months because the mirror tilt angle of Gen2.50.10 matched the low elevation angle of the sun.

A fixed model configuration will receive different amounts of irradiance throughout a year. The "gullwing curve" phenomenon is not ideal for yearly power production because the summer is the best time for power production. The fixed MAPV system should not only maximize yearly power production, but also try to obtain maximum power output every month. A different model is necessary to fully capitalize on the benefits of increased power production. Therefore, a further design of MAPV is necessary.

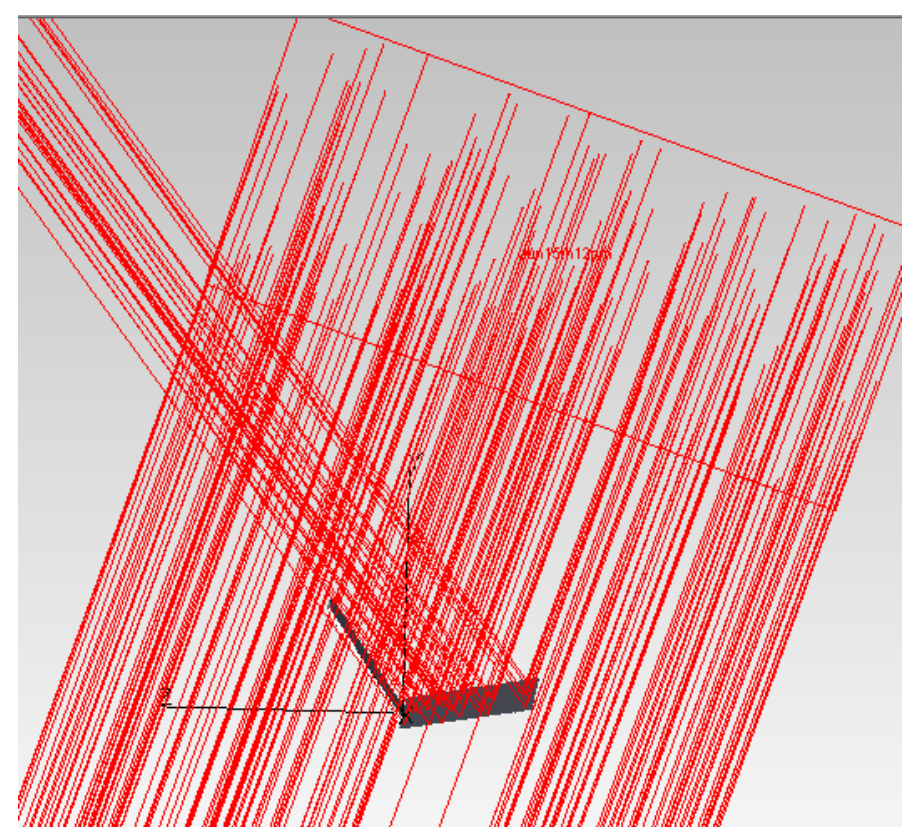

Figure 43: The ray-trace result for the Gen2.50.10 model during summer.

\subsection{The Effect of Mirror Tilt Angle on Uniformity and Power Production}

The sun's elevation angles are the lowest in the winter and increase to the highest point in the summer. The elevation of the sun is an important parameter for the model 
configuration. The fixed MAPV can maintain good uniformity in most of the models while the sun's elevation angles are high (summer), but only few models can maintain good conditions at low elevation angles (winter). The model configuration should be tuned in order to obtain a uniform irradiance distribution and maximum power output throughout the year, but ideally could maintain maximum power output for both the summer and winter months. The Gen2.55.0 was the best model configuration to harvest most sunlight in January because of the low mirror tilt angle due to the sun being at a low elevation. The best mirror tilt angle for the summer was $30^{\circ}$ because it matches the sun's elevation angle very well. The reflected light can fully illuminate on the desired PV panel. The gullwing curves exist on most of the model configurations listed in Table 5 (Figure 37). This indicates that higher mirror tilt angle is effective for harvesting sunlight with high elevation angle of the sun (summer). For example, Figure 44 shows the ray-trace result for the Gen2.55.30 model in the summer, it can be confirmed that the reflected light can be directed right on the PV panel due to high mirror tilt angle. The reflected light of Gen2.55.10 model (Figure 43) was directed off the PV panel in the summer. The ray-trace results from the Gen2.55.10 through the Gen2.55.30 models (Table 5) show that a higher tilt angle is more effective at harvesting sunlight when the sun is at a high elevation angle (i.e. summer). Therefore, the best MAPV system design for increasing power production in the summer months would have a high mirror tilt angle, but this would not help increase power during the winter months. The mirror tilt angle was an important parameter in determining the best model design for a particular MAPV system based on what need the MAPV system is trying to fill. In this section, it can be concluded 
that the fixed MAPV does not maintain high energy output for entire year due to the mismatch of the relative position between the mirror tilt angle and the sun.

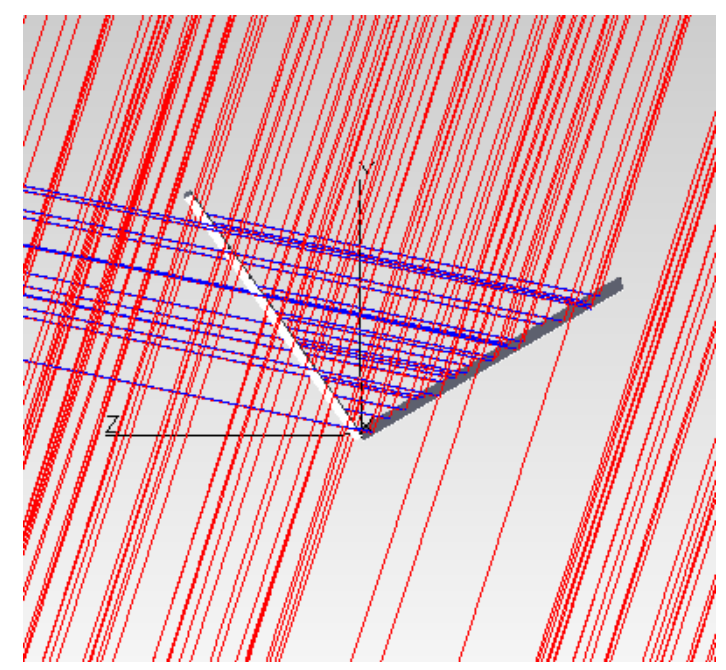

Figure 43: The ray-trace result for the Gen2.55.30 model on June $15^{\text {th }}, 2012$ at noon.

\subsection{Viability of Different Mirror Designs}

Section 4.7 introduced two kinds of mirror designs for solving the "gullwing curve" effect. The first concept was to model a barrel mirror design. Gen3.55.0.90 showed better uniformity compared to Gen3.55.0.540; however, the low radius of curvature of Gen3 model reflected significant portion of the light away from the PV module (Figure 39). That is why Gen3.55.0.540 model obtained a higher irradiance (Figure 40). Comparing both the results to Gen2 model in Table 5, Gen 3 models have lower irradiance value and worse uniformity. The barrel mirror not only reflects light away from the PV module, but also destroys the uniform irradiance distribution of Gen2 model.

A concave mirror was designed to concentrate sunlight onto the PV panels. The Gen4 models do successfully harvest more light compared to Gen2 model in Table 5 because the concave mirror concentrates the light very well. Nevertheless, Gen4 models 
are more non-uniform than Gen3 models (Figure 39-42). As a result, Gen4 might be a good option for the purpose of higher energy output, but not for better uniformity of light distribution. Gen3 models cannot fulfill both the requirements (high power output and good uniformity) needed. A better mirror design is needed to satisfy these two requirements.

A new possible mirror design was to use a wider concave mirror with high radius of curvature. The wider mirror helps to extend the mirror-augmented region, which leads to higher power output. The concave mirror with a high radius of curvature helps to obtain uniform irradiance distribution. The cost of fabricating the new mirror type needs to be understood compared to the increase in power production of the fixed MAPV system. Another possible solution is to change the PV module from crystalline silicon panels to thin film panels. The thin film solar cell does not have the problem of the power loss while the by-pass diodes operating. However, the comparable disadvantages for thin film PV modules are increased cost and reduced efficiency.

\section{Conclusion}

Photovoltaic electricity has the potential to serve as a competitive and efficient energy source in the future. However, the prime cost of this technology is still higher than nuclear, thermal and wind power. One simple and effective way to drive down the cost of PV electricity is to combine reflectors with PV panels in order to harvest more light from the modules. In this study, an optimized design configuration was used to analyze the performance of a fixed (non-tracked) MAPV system. Non-uniform illumination patterns caused by both shadowing effects and mirror augmentation were observed. The simulated 
mirror-augmented systems also experienced "end effects" during which the panel was not fully augmented because of inadequate mirror lengths. By modifying the length of mirror, the "end effect" in the mirror-augmented model can be removed.

The optical analysis and experimental I-V data both show that the MAPV system has higher power output compared to a non-augmented PV system. The experimental illumination patterns match ray-trace predictions well and validate the optical accuracy of our model. However, the power production from field-test is lower than expected due to assumptions made in the simulations such as perfect absorber of PV panel, 88\% reflectivity of solar mirror. The first prototype of fixed MAPV shows a $10 \%$ boost over standard PV during time-limited period. This result shows that the fixed MAPV system is feasible to reduce the cost of power generated by PV by using inexpensive solar mirror. The fixed MAPV system will need to overcome the non-uniformity issue caused by mirror-augmentation.

The optimal condition for a fixed MAPV is to fully illuminate the reflected light on PV panel for the entire year. Under these conditions, the fixed MAPV becomes a worthwhile investment for a power provider. A "gullwing curve" was found in the estimated annual power of the fixed MAPV system models. The mirror tilt angle should be kept low in winter to obtain the highest power output and good irradiance uniformity. With the higher sun's elevation angles (summer months), the mirror can be tilted to higher angle for harvesting more light and also maintaining uniformity. Further fixed MAPV system designs need to be investigated in order to maximize the power production produced with a fixed MAPV system for an entire year. 


\section{Bibliography}

${ }^{1}$ The information of solar energy history provided by Zero Point 4 Energy, [online] (2012, Dec.). Available: http://www.zero-point4energy.com/solar-energy-history.html

${ }^{2}$ The information of energy crisis provided by Wikipedia, [online] (2012, Dec.). Available: http://en.wikipedia.org/wiki/Energy_crisis

${ }^{3}$ J. Klein, A. Rednam, D. Ashuckian, S. Bender, B. B. Blevins, "Comparative costs of California central station electricity generation technologies", California Energy Commission, June 2007.

${ }^{4}$ R. H. French, M. P. Murray, W. C. Lin, K. A. Shell, S. A. Brown, M. A. Schuetz, R. J. Davis, "Solar radiation durability of materials components and systems for low concentration photovoltaic systems”, IEEE Energytech, Cleveland, USA, May 2011.

${ }^{5}$ The information of solar mirror provided by Replex Plastics Corp., Mount Vernon, OH [online] (2012, Feb.). Available: http://www.replex.com/?id=applications\&pg=solar

${ }^{6}$ M. A. Schuetz, K. A. Shell, S. A. Brown, G. S. Reinbolt, R. H. French, R. J. Davis, "Design and construction of a $\sim 7 \mathrm{x}$ low-concentration photovoltaic system based on compound Parabolic concentrators”, IEEE Journal of Photovoltaics, 2012

${ }^{7}$ R. H. French, J. M. Rodríguez-Parada, M. K. Yang, R. A. Derryberry, N. T. Pfeiffenberger, “Optical Properties Of Polymeric Materials For Concentrator Photovoltaic Systems” Sol. Energy Mater. Sol. Cells, 95, 2077-86, (2011),doi:10.1016/j.solmat.2011.02.025. 
${ }^{8}$ Myles P. Murray, Devin Gordon, Scott A. Brown, Wei-Chun Lin, Kara A. Shell, Mark A. Schuetz, Sean Fowler, Jim Elman, Roger H. French, "Solar Radiation Durability Framework Applied To Acrylic Solar Mirrors”, Proceedings of the SPIE Solar Energy + Technology: Reliability of Photovoltaic Cells, Modules, Components, and Systems IV, San Diego, August 21-25, 2011.

${ }^{9}$ Myles P. Murray, Laura S. Bruckman, Roger H. French, "Durability of Materials in a StressResponse Framework: Acrylic Materials for Photovoltaic Systems”, Materials Research Society Proceedings, December 2011.

${ }^{10}$ Myles P. Murray, Laura S. Bruckman, Roger H. French, "Photodegradation in a Stress and Response Framework: Poly(methyl methacrylate) for Solar Mirrors and LensPhotodegradation in a Stress and Response Framework: Poly(methyl methacrylate) for Solar Mirrors and Lens" in press to Journal of Photonics for Energy.

11 The information of motions of the sun simulator provided by Astronomy Education at the University of Nebraska-Lincoln, [online] (2012, Dec.). Available: http://astro.unl.edu/naap/motion3/animations/sunmotions.swf

12 C. Deline, "Photovoltaic shading test for module-level power electronics", NREL, Tech. Rep. NREL/TP-5200-54876

${ }^{13}$ K. Butti, J. Perlin, “The history of terrestrial uses of solar energy”, Solar Energy Handbook, McGraw-Hill, New York, 1981

${ }^{14}$ D. Chapin, C. Fuller, G. Pearson, J. Appl. Phys. 25, 676, 1954 
${ }^{15}$ R. M. Swanson, "Photovoltaic concentrators", Handbook of photovoltaic science and engineering, SunPower Corp., Sunnyvale, CA

${ }^{16}$ C. Pfeifer, P. Schoffer, B. Spars, J. Duffie, Trans. ASME, J. Eng. Power January, 33-38, 1962

${ }^{17}$ W. Beckman, P. Schoffer, Jr. W. Hartmann, G. Lof, Sol. Energy 10, 132-136, 1966

18 Rosenthal, A.L. and C.G. Lane, Field Test Results for the 6 MW Carrizo Solar Photovoltaic Power Plant. Solar Cells, 1991. 30: p. 563-571.

19 Gay, C.F. and E. Berman, Performance of large photovoltaic systems. Chemtech, 1990.

${ }^{20}$ A. W. Czandema, G. J. Jorgensen, “Accelerated life testing and service lifetime prediction for PV technologies in the twenty-first century", NREL, 1999

${ }^{21}$ A. L. Rosenthal, G. G. Lane, "Field test results for the $6 \mathrm{MW}$ carrizo solar photovoltaic power plant”, Solar Cells, 30, 563-571, 1991

${ }^{22}$ Kemp, M., et al., Polymeric Testing Consideration for Photovoltaic Applications. NIST/UL Workshop on Polymer for Photovoltaic Applications, 2010

${ }^{23}$ M. I. Smokler, D. H. Otth, R. G. Ross, Jr., "The block program approach to photovoltaic module development", Jet Propulsion Laboratory, IEEE 1985

${ }^{24}$ M. Kempe, T. Moricone, M. Kilkenny, "Effects of cerium removal from glass on photovoltaic module performance and stability", National Renewable Energy Laboratory, Golden, Colorado, Conference Paper NREL/CP-530-44936, September 2009. 
${ }^{25}$ P. Klemchuk, M. Ezrin, G. Lavgne, W. Holley, J. Galica and S. Agro, "Investigation of the degradation and stabilization of EVA-based encapsulant in field-aged solar energy modules", Polymer Degradation and Stability, 55, 347-365, 1997

${ }^{26}$ M. Kempe, "Evaluation of encapsulant materials for PV applications”, www.pv-tech.org, NREL, Colorado 2011

${ }^{27}$ The 1D model calculations achieved by Dave Hollingshead at Replex Plastics, March, 2012.

${ }^{28}$ The information of MatLab provided by MathWorks, [online] (2012, Dec.). Available: http://www.mathworks.com/products/matlab/

29 Ibrahim Reda, and Afshin Andreas, "Solar position algorithm for solar radiation applications," National Renewable Energy Laboratory, Golden, Colorado, Tech. Rep. NREL/TP-560-34302, Revised Jan. 2008.

${ }^{30}$ Kasten, F. Young, AT, "Revised optical air mass tables and approximation formula," Applied Optics [internet]. 1989 Available from: http://ao.osa.org/abstract.cfm?URI=ao$\underline{28-22-4735}$

${ }^{31}$ The information of air mass provided by PV Education, [online] (2012, Dec.). Available: http://www.pveducation.org/pvcdrom/properties-of-sunlight/air-mass

${ }^{32}$ The information of the raw incident panel intensity provided by PV Education, [online] (2012, Dec.). Available: http://www.pveducation.org/pvcdrom/properties-ofsunlight/arbitrary-orientation-and-tilt 
33 The information of Sun's position to high accuracy provided by PV Education, [online] (2012, Dec.). Available: http://www.pveducation.org/properties-of-sunlight/sunposition-high-accuracy

${ }^{34}$ P. Benitez, J. L. Aivarez, J. C. Minano, "Recent developments on the flat-plat microconcentrator module", IEEE 2002

${ }^{35}$ W. C. Fei, C. H. Huang, W. C. Hsu, J. C. Tsai, "Design and simulation of a secondary solar concentrator constructed with a circular micro prism array for the enhancement of the concentration ratio", IEEE 2009

${ }^{36}$ H. Y. Pan, S. H. Chang, B. H. Ke, K. J. Chen, “The study on the allowing angle of the sun's rays of concentrated photovoltaic concentrator", IEEE 2011

${ }^{37}$ Y. Ota, K. Nishioka, “3-dimensional simulator for concentrator photovoltaic modules using ray-trace and circuit simulator", IEEE 2010

${ }^{38}$ Y. Ota, K. Nishioka, "Total simulator for concentrator photovoltaic modules using ray-trace and circuit simulators", IEEE 2009

39 The information of TracePro provided by Lambda Research Corp., [online] (2012, Dec.). Available: http://lambdares.com/software_products/tracepro/

40 The Software of TracePro provided by Lambda Research Corp., [online] (2012, Dec). Available: http://lambdares.com/

${ }^{41}$ The information of SolidWorks provided by Dassault Systemes SolidWorks Corp., [online] (2012, Dec.). Available: http://www.solidworks.com/ 
${ }^{42}$ The 3D model constructed by Kara A. Shell at Replex Plastics, January, 2012

${ }^{43}$ The information of national solar radiation data base provided by NREL, [online] (2012, Dec.). Available: http://rredc.nrel.gov/solar/old_data/nsrdb/1991-2005/tmy3/

${ }^{44}$ The information of national solar radiation data base provided by NREL, [online] (2012, Dec.). Available: http://rredc.nrel.gov/solar/old_data/nsrdb/1991-2005/tmy3/

45 The information of Multi-tracer provided by Daystar Corp., Las Cruces, NM [online] (2012, Feb.). Available: http://www.zianet.com/daystar/multitracer.html

${ }^{46}$ The information of Pyranometer CMP6 provided by Kipp\&Zonen Corp., Netherlands [online] (2012, Feb.). Available: http://www.kippzonen.com/?product/1251/CMP+6.aspx

${ }^{47}$ The information of CS-6P provided by CSI Corp., Ontario, Canada [online] (2012, Feb.). Available: http://solarinstallco.com/images/cs6p200.pdf

${ }^{48}$ The information of Gen1 MAPV model provided by Dovetail Solar and Wind, [online] (2012, Dec.). Available: http://www.dovetailsolar.com/About-Us/About-Us.aspx

49 The Daystar data from field-test site at Replex Plastics acquired by Dave Hollingshead, May, 2012. 
${ }^{50}$ L. Bruckman, M. Murray, S. R. Murray, R. French, S. Brown, M. Schuetz, "Degradation of back surface acrylic mirrors: implications for low concentration and mirror augmented photovoltaics”, IEEE EnergyTech, 2012 